\title{
On the Possibility of [1,5] Sigmatropic Shifts in Bicyclo[4.2.0]octa-2,4-dienes
}

Hannelore Goossens, ${ }^{a}$ Johan M. Winne, ${ }^{b}$ Sebastian Wouters, ${ }^{a}$ Laura Hermosilla, ${ }^{c}$ Pierre J. De Clercq, ${ }^{\mathrm{b}}$ Michel Waroquier, ${ }^{\mathrm{a}}$ Veronique Van Speybroeck ${ }^{\mathrm{a}}$ and Saron Catak $^{\mathrm{a}, \mathrm{d}_{*}}$

${ }^{a}$ Center for Molecular Modeling, Ghent University, Technologiepark 903, 9052 Zwijnaarde, Belgium

${ }^{b}$ Department of Organic and Macromolecular Chemistry, Ghent University, Krijgslaan 281/S4, 9000 Ghent, Belgium

${ }^{c}$ Departamento de Química Física Aplicada, Universidad Autónoma de Madrid, 28049 Madrid, Spain ${ }^{d}$ Department of Chemistry, Bogazici University, 34342 Bebek, Istanbul, Turkey²

Keywords: Endiandric acids, bicyclo[4.2.0]octa-2,4-dienes, electrocyclic conversion, [1,5] sigmatropic shift, walk rearrangement, DFT, DMRG and CASSCF calculations

\section{TOC Graphic}

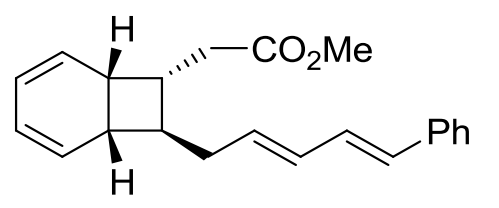

Endiandric Acid D methyl ester
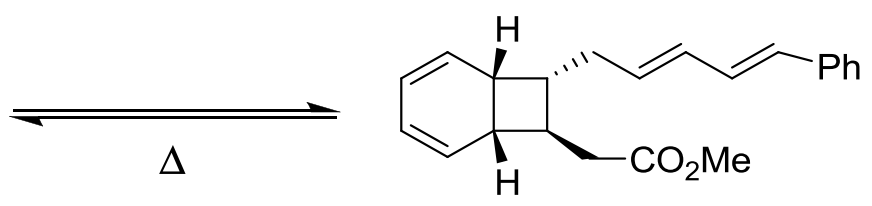

Endiandric Acid E methyl ester

\begin{abstract}
The thermal equilibration of the methyl esters of endiandric acids D and E was subject to a computational study. An electrocyclic pathway via an electrocyclic ring opening followed by a ring flip and a subsequent electrocyclization proposed by Nicolaou [Chem. Soc. Rev. 2009], was computationally explored. The free energy barrier for this electrocyclic route was shown to be very close to the bicyclo[4.2.0]octa-2,4-diene reported by Huisgen [Tet. Lett. 1968].
\end{abstract}


Furthermore, the possibility of a [1,5] sigmatropic alkyl group shift of bicyclo[4.2.0]octa-2,4diene systems at high temperatures was explored in a combined computational and experimental study. Calculated reaction barriers for a biradical-mediated stepwise [1,5] sigmatropic alkyl group shift were shown to be comparable with the reaction barriers for the bicyclo[4.1.0]hepta2,4-diene (norcaradiene) walk rearrangement, whereas calculated reaction barriers for a concerted [1,5] sigmatropic alkyl group shift were found to be higher in energy. However, the stepwise pathway is suggested to only be feasible for appropriately substituted compounds. Experiments conducted on a deuterated analogous diol derivative confirmed the calculated (large) differences in barriers between electrocyclic and sigmatropic pathways.

\section{INTRODUCTION}

Endiandric acids, phytochemicals that were first discovered by Gatehouse and Black, ${ }^{1,2}$ and their derivatives possess various biological activities, ${ }^{3}$ such as antibacterial, ${ }^{4-6}$ antitubercular ${ }^{7}$ and anticancer properties. ${ }^{6,8}$ Their biosynthesis via an intricate cascade of pericyclic reactions was proposed by Black ${ }^{2}$ and verified experimentally by Nicolaou. ${ }^{8-12}$ As part of the biomimetic synthesis, Nicolaou described an unexpected thermal equilibrium between two bicyclo[4.2.0]octa-2,4-diene intermediates - the methyl esters of the natural products endiandric acid D and endiandric acid E - (Scheme 1, compounds $\mathbf{1}$ and 2, respectively) and proposed a three step electrocyclic cascade for this equilibrium via (a) an electrocyclic ring opening followed by (b) a ring flip of the resulting cyclooctatriene (COT) and (c) a subsequent electrocyclization. 

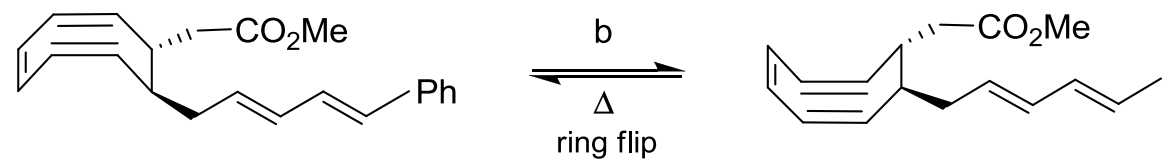

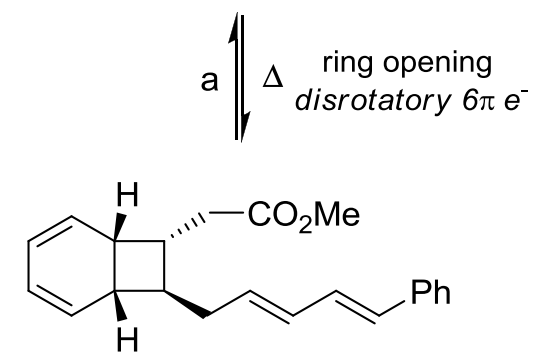

Endiandric Acid D methyl ester

1

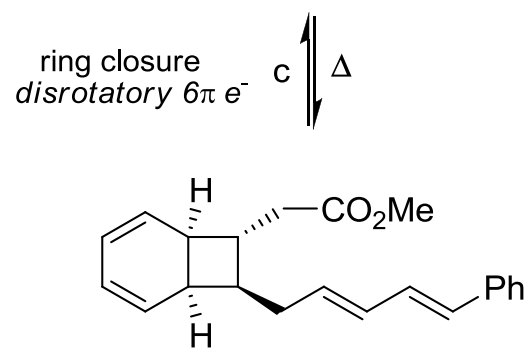

Endiandric Acid E methyl ester

2

Scheme 1. Thermal rearrangement of Endiandric acids D and E: The electrocyclic route.

However, an alternative sigmatropic mechanism for this thermal rearrangement via a $[1,5]$ carbon shift might be possible at high temperatures (Scheme 2).

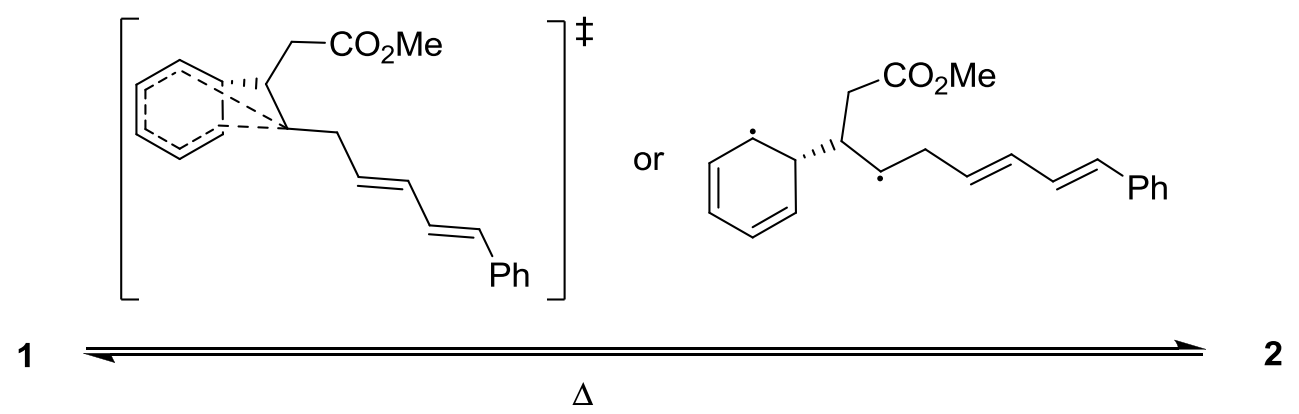

Scheme 2. Thermal rearrangement of Endiandric acids D and E via a concerted sigmatropic route (transition state on the left) or a stepwise sigmatropic route (biradical intermediate on the right).

Most sigmatropic [1,5] hydrogen migrations (Scheme 3, I, R=H) are pericyclic transformations, which typically possess relatively high activation barriers and thus usually require high reaction temperatures. ${ }^{13-15}$ Pericyclic reactions are important both from a synthetic and a theoretical point of view, ${ }^{16-17}$ due to their highly ordered transition states, these concerted transformations usually offer a high degree of selectivity and a high level of mechanistic insight. ${ }^{18-19}$ Different types of observed and hypothetical pericyclic processes have been very efficiently categorized depending 
on the nature of the interacting molecular orbitals. Moreover, consideration of the required symmetry of the implicated orbitals leads to a straightforward prediction of a specific transformation being "favored" or "disfavored". ${ }^{20-23}$ However, whether a pericyclic process is a viable reaction pathway, depends on a complex interplay of many factors, and therefore, it is often difficult to make reliable predictions.

(I)

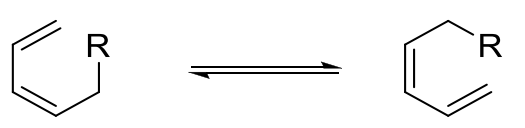

(II)<smiles>c1ccccc1</smiles>

3

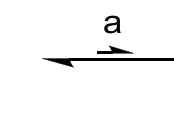

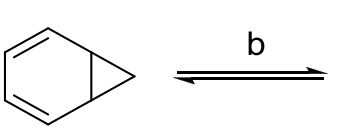

4

$$
\mathrm{R}=\mathrm{H} ; \mathrm{E}_{\mathrm{a}}=30-40 \mathrm{kcal} / \mathrm{mol}^{13}
$$$$
\mathrm{R}=\mathrm{alkyl} ; \mathrm{E}_{\mathrm{a}}=35-40 \mathrm{kcal} / \mathrm{mol}^{13}
$$<smiles>c1ccc2c(c1)CC2</smiles>

$\mathrm{E}_{\mathrm{a}}(4 \rightarrow 5)=35-45 \mathrm{kcal} / \mathrm{mol}^{38,39}$

(III)<smiles>C1=CC=CCCC=C1</smiles>

6

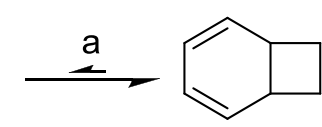

7

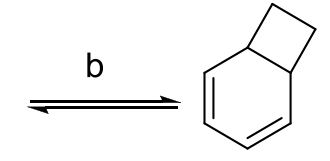

8

Scheme 3. [1,5] Sigmatropic rearrangements of: (I) 1,3-dienes; (II) bicyclo[4.1.0]hepta-2,4dienes; (III) bicyclo[4.2.0]octa-2,4-dienes.

Sigmatropic $[1, n]$ carbon migrations (Scheme 3, I, R=alkyl) on the other hand, do not generally involve concerted transition states because the overlap of the orbitals in the transition structure is usually too weak, ${ }^{24}$ but are believed to occur via intermediate singlet-state biradicals. ${ }^{25,26}$ Exceptions, involving pericyclic transition states with good overlap, are the [1,5] sigmatropic migration in 1,3-cyclopentadienes ${ }^{27-28}$ and the so-called "walk rearrangements"29-33 of bicyclo[n.1.0]polyenes for which the thermally allowed process should occur with inversion of configuration at the migrating carbon atom. ${ }^{26,34-35}$ Walk rearrangements are [1,5] sigmatropic 
shifts which involve the migration of a divalent group $\left(\mathrm{O}, \mathrm{S}, \mathrm{NR}\right.$ or $\left.\mathrm{CR}_{2}\right)$ that is part of a threemembered ring in a bicyclic system (Scheme 3, II, for $\mathrm{CH}_{2}$ ). These thermally induced processes have been demonstrated in various bicyclo[n.1.0]polyene structures.

Thermal rearrangements of bicyclo[4.1.0]hepta-2,4-diene 4 (or norcaradiene, Scheme 3, II) systems have received a lot of attention in both experimental and computational studies, ${ }^{16,30-31,36-}$ 39 as they have been observed to proceed with inversion at the migrating center, indicating an orbital-symmetry forbidden rearrangement. ${ }^{26,40,41}$ However, these reactions have been shown not to be concerted and thus not subject to the rules of orbital symmetry conservation. ${ }^{26}$

The experimentally determined activation energies for various substituted norcaradiene walk rearrangements do not differ significantly from those of normal [1,5] alkyl shifts (Scheme 3, II and I with $\mathrm{R}=$ alkyl, respectively). ${ }^{38}$ This can be rationalized by the fact that the norcaradiene system 4 is usually the less populated valence tautomer in a $6 \pi$ electrocyclization equilibrium with a less constrained cycloheptatriene $\mathbf{3}$ (IIa), adding to the overall barrier for the carbon shift. However, a different situation exists for the homologous bicyclo[4.2.0]octa-2,4-diene (Scheme 3, III), where the electrocyclization product 7 is known to be favored over the contorted cyclooctatriene 6 form in most cases (IIIa). ${ }^{42}$

Although there is no prior literature of walk rearrangements in ethylene bridged cyclic polyene systems, in the context of the well-documented similarity in the reactivity of vinyl cyclopropane and vinyl cyclobutane systems in their formal $[1,3]$ carbon shifts to a cyclopentene and a cyclohexene system, respectively (Scheme 4, I and II, respectively), ${ }^{43-47}$ at high temperatures, a ring walk-type [1,5] carbon shift in a bicyclo[4.2.0] octa-2,4-diene (Scheme 3, IIIb) system seems to be a viable reaction pathway on the basis of the norcaradiene precedent. 


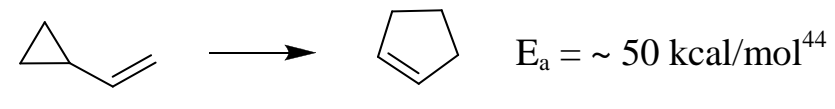

(II)

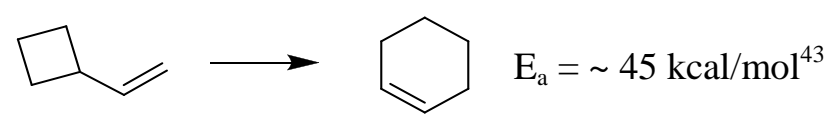

Scheme 4. [1,3] Sigmatropic rearrangements of (I) vinyl cyclopropane and (II) vinyl cyclobutane.

As there is no straightforward way to distinguish experimentally between these two mechanistic schemes (electrocyclic versus sigmatropic) in this particular case, both rearrangement pathways have been comparatively studied from a theoretical point of view.

Additionally, in order to verify theoretical results, an experiment using a model bicyclo[4.2.0]octa-2,4-diene system 9 (Scheme 5) has been devised. Due to the pseudo- $\mathrm{C}_{2-}$ symmetry of this system, the interconverting structures (with respect to their Endiandric Acid D and E counterparts) are identical (Scheme 5, a). However, this model system is readily accessible as the deuterium labeled analog $\mathbf{9 - d _ { 4 }}$. The thermal rearrangement of diol $\mathbf{9 - \mathbf { d } _ { 4 }}$ would only be unnoticed if it proceeds exclusively via the electrocyclic route. A sigmatropic pathway (or walk rearrangement) would lead to different products with respect to their deuterium substitution patterns (Scheme 5, b). 
(a)<smiles>OC[C@H]1C[C@H]2C=CC=C[C@H]2[C@H]1CO</smiles>

9 electrocyclic<smiles></smiles>

sigmatropic

$[1,5]$<smiles>OC[C@H]1C[C@H]2C=CC=C[C@H]2[C@H]1CO</smiles>

$10=9$ (b)

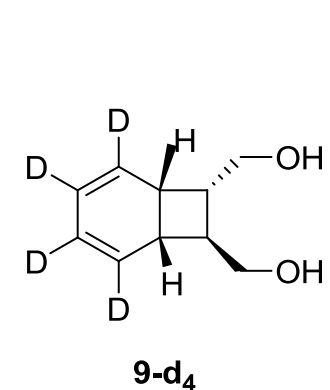

9- $d_{4}$

$\rightleftharpoons$

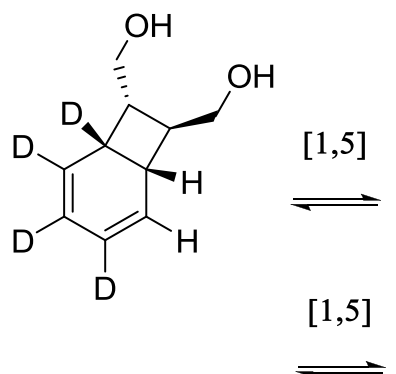

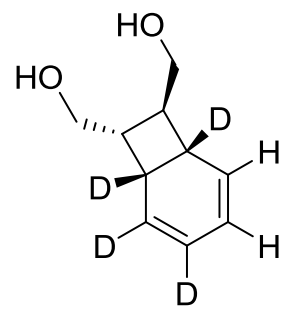

6 possible $9-d_{4}$
isotopomers

Scheme 5. Thermal rearrangements of a model diol system 9 (a) and its deuterium-labeled analog 9-d $(b)$.

Thus, the aim of this study is two-fold; unraveling the mechanism of thermal equilibration between endiandric acid methyl esters $\mathrm{D} / \mathrm{E}$ in particular and more generally exploring the possibility of [1,5] sigmatropic alkyl shifts (walk rearrangements) in bicyclo[4.2.0]octa-2,4-diene systems at high temperatures through a combined computational and experimental study.

\section{COMPUTATIONAL METHODOLOGY}

All reactants, transition states, intermediates and products were optimized using three different functionals with a $6-31+\mathrm{G}(\mathrm{d}, \mathrm{p})$ basis set: ${ }^{48-49}$ the well-established hybrid functional B3LYP, ${ }^{50-51}$ Truhlar's meta hybrid exchange-correlation functional M06-2X, ${ }^{52-53}$ which accounts for dispersion and Grimme's B3LYP-D3 approach, ${ }^{54}$ which takes into account van der Waals interactions by empirically adding long-range dispersive corrections. Harmonic vibrational frequencies were computed at the same levels of theory and used to provide thermal corrections to the Gibbs free energies and to confirm the nature of the stationary points. The intrinsic reaction coordinate (IRC) ${ }^{55-56}$ paths were traced to verify the two associated minima connected to 
each transition state on the potential energy surfaces. In order to investigate the possibility of open-shell transition states and an open-shell biradical intermediate for the sigmatropic processes, HOMO and LUMO initial guesses were mixed to produce unrestricted wavefunctions for singlet states and the stability of the wavefunctions was checked. ${ }^{57-58}$ These calculations were carried out with the Gaussian 09 program package.$^{59}$ In order to assess the diradical character, CASSCF/6-31+G(d,p) calculations were carried out for the structures optimized with M06-2X. An active space of ROHF molecular orbitals with all valence electrons was targeted with the density matrix renormalization group (DMRG) ${ }^{60-61}$ which yielded approximate natural orbitals. Based on the natural orbital occupation numbers (NOON), the active space for the subsequent CASSCF calculations was identified: natural orbitals with $0.01<$ NOON $<1.99$ were regarded as essential for the CASSCF calculations. We refer the reader to Ref. 62 for an introduction to this procedure, which yields an unbiased initial orbital guess. Both the DMRG and CASSCF calculations were carried out with the free open-source ab initio DMRG code CHEMPS2 ${ }^{63-64}$ For the initial DMRG rotation to approximate natural orbitals, $D_{\mathrm{SU}(2)}=750$ reduced renormalized basis states were retained. In order to obtain Gibbs free CASSCF energies, thermal free energy corrections were taken from the M06-2X optimizations.

\section{RESULTS AND DISCUSSION}

Electrocyclic and sigmatropic pathways were computationally explored for the thermal equilibration of three different bicyclo[4.2.0]octa-2,4-diene systems. Computational results were compared with relevant literature data where applicable. The possibility of $[1,5]$ sigmatropic alkyl shifts (walk rearrangements) at high temperatures was also experimentally explored. 


\section{Thermal Equilibration of Bicyclo[4.2.0]octa-2,4-diene 9}

The thermal equilibration of bicyclo[4.2.0]octa-2,4-diene 9 via electrocyclic and sigmatropic (concerted and stepwise) pathways was explored in a combined computational and experimental study.

\section{A. Theoretical study}

Initially, an electrocyclic pathway via an electrocyclic ring opening followed by a ring flip and a subsequent electrocyclization, which was proposed by Nicolaou for endiandric acids D and E, ${ }^{8-12}$ was studied computationally for bicyclo[4.2.0]octa-2,4-diene 9 (Figure 1, pathway a).

Orbital symmetry selection rules state that "allowed" sigmatropic reactions occur through concerted pathways, as opposed to "forbidden" processes that are known to thermally occur via stepwise pathways, which go through biradical intermediates. ${ }^{65}$ However, it has been shown that stepwise routes may be favored over concerted ones for some orbital symmetry allowed processes, where substituents stabilize the intermediate biradical. ${ }^{18,66-68}$ For this reason, the thermal [1,5] sigmatropic carbon shift under study has been explored through both a concerted and a biradical-mediated stepwise pathway (Figure 1, pathways $b$ and $c$, respectively). 
ELECTROCYCLIC PROCESS

(a)

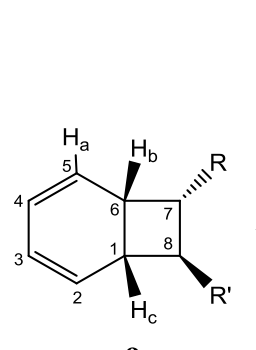

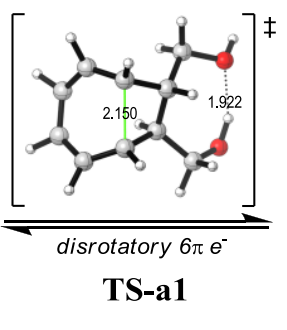

Int-a1

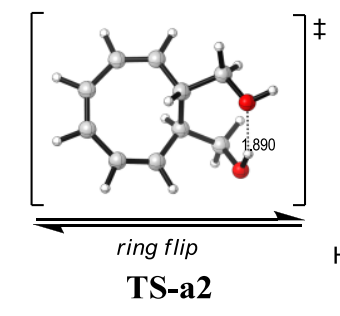

TS-a2

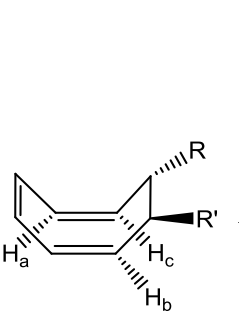

Int-a2

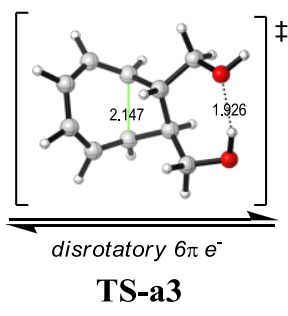

TS-a3

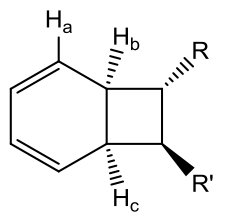

10

\section{SIGMATROPIC PROCESS}

(b)

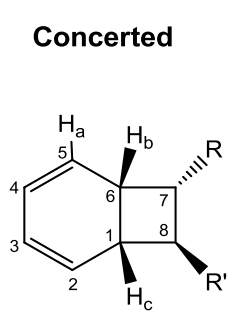

9

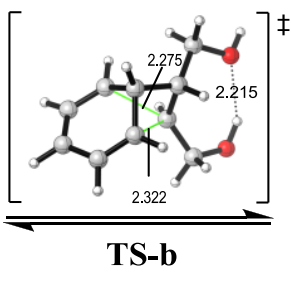

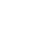

Stepwise

(c)

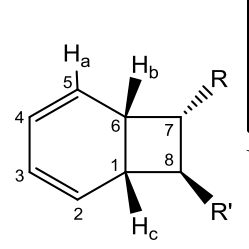

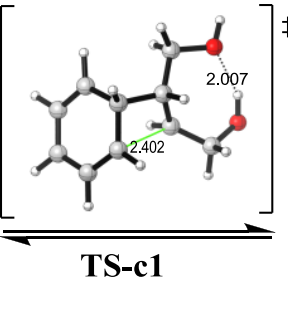

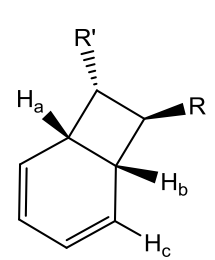

10

Figure 1. Schematic representation of the electrocyclic and sigmatropic mechanisms for the thermal equilibration of

bicyclo[4.2.0]octa-2,4-diene $9 .^{\mathrm{a}-\mathrm{c}}$

${ }^{\mathrm{a}} \mathrm{R}=\mathrm{R}^{\prime}=-\mathrm{CH}_{2} \mathrm{OH} .{ }^{\mathrm{b}} \mathrm{M} 06-2 \mathrm{X} / 6-31+\mathrm{G}(\mathrm{d}, \mathrm{p})$ geometries for pathway $a$ and UM06-2X/6-31+G(d,p) geometries for pathways $b$ and $c .{ }^{\mathrm{c}}$ Distances in $\AA$. 


\section{Electrocyclic Conversion of Bicyclo[4.2.0]octa-2,4-diene 9}

Figure 1 depicts a schematic representation along with optimized transition state geometries for the electrocyclic pathway of the thermal equilibration of bicyclo[4.2.0]octa-2,4-diene 9 (pathway a). Furthermore, the free energy profile is shown in Figure 2. The first step in the electrocyclic process is the ring opening of $\mathbf{9}$ via C1-C6 bond cleavage through transition state TS-a1. This early transition state has a C1-C6 bond elongation that is relatively small (2.150 $\AA$ compared to $1.558 \AA$ and $3.081 \AA$ for reactant 9 and intermediate Int-a1, respectively) and the Gibbs free activation barrier $\left(\Delta G^{\ddagger}\right)$ for this step is $26.0 \mathrm{kcal} / \mathrm{mol}$ at the $\mathrm{M} 06-2 \mathrm{X} / 6-31+\mathrm{G}(\mathrm{d}, \mathrm{p})$ level of theory. The ring opening leads to a contorted cyclooctatriene intermediate Int-a1, which subsequently undergoes a ring flip through transition state TS-a2. This second step is characterized by a $\Delta G^{\ddagger}$ of only $6.6 \mathrm{kcal} / \mathrm{mol}$. Finally, electrocyclization through transition state TS-a3 $\left(\Delta G^{\ddagger}=21.6\right.$ $\mathrm{kcal} / \mathrm{mol}$ ) generates product $\mathbf{1 0}$, which is identical to the starting compound $\mathbf{9}$ due to symmetry. However, retention of the hydrogen bond during the reaction causes a subtle energy difference between $\mathbf{9}$ and $\mathbf{1 0}$ at some levels of theory, which is also the case for TS-a1 and TS-a3, and Inta1 and Int-a3. 


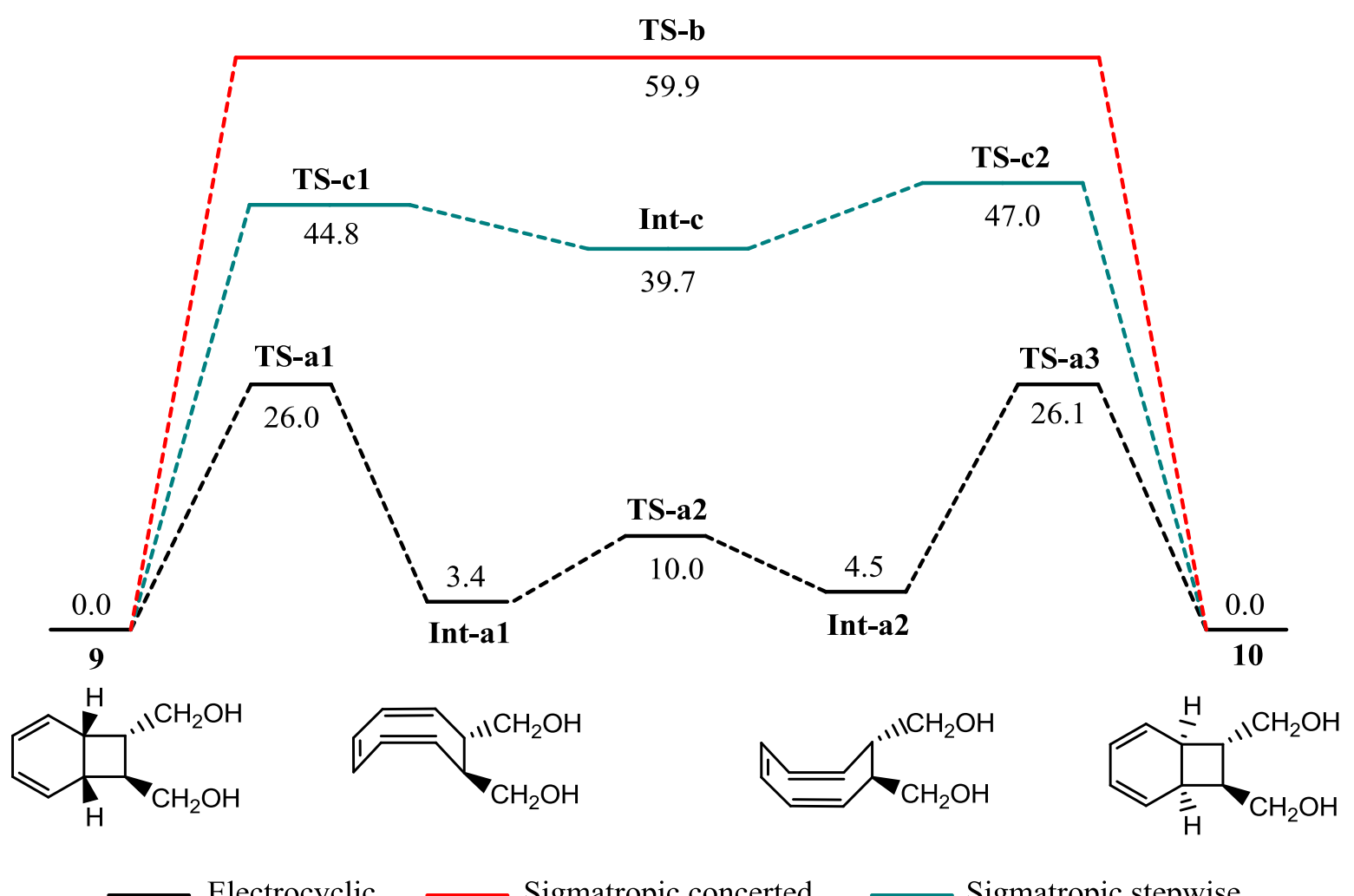

Figure 2. Free energy profile for the electrocyclic $(\mathrm{M} 06-2 \mathrm{X} / 6-31+\mathrm{G}(\mathrm{d}, \mathrm{p}))$ and sigmatropic pathways (UM06-2X/6-31+G(d,p)) for the thermal equilibration of bicyclo[4.2.0]octa-2,4-diene

9. Energies in $\mathrm{kcal} / \mathrm{mol}$.

\section{[1,5] Sigmatropic Alkyl Shift of Bicyclo[4.2.0]octa-2,4-diene 9}

The sigmatropic alkyl group shift could take place via a concerted mechanism, where C1-C8 bond cleavage, rotation of the migrating carbon around the C6-C7 bond and formation of the new bond (C5-C8) take place in a synchronous concerted fashion (Figure 1, pathway b). In transition state TS-b, the C1-C8 bond is elongated (C1-C8 distance $2.322 \AA)$ and a slight twist around the $\mathrm{C} 6-\mathrm{C} 7$ bond results in an optimal position to form the new bond (C5-C8 distance $2.275 \AA$ ). The activation energy for this concerted sigmatropic process is very high $\left(\Delta G^{\neq}=59.9\right.$ $\mathrm{kcal} / \mathrm{mol}$, UM06-2X/6-31+G(d,p), Figure 2). Alternatively, the sigmatropic alkyl group shift 
could take place via a biradical-mediated stepwise mechanism (Figure 1, pathway c), where the first step consists of homolytic C1-C8 bond cleavage and subsequent rotation through transition state TS-c1 to the biradical intermediate Int-c. The transition state for this step has a Gibbs free activation barrier $\Delta G^{\ddagger}$ of $44.8 \mathrm{kcal} / \mathrm{mol}$, which is lower than that for the concerted sigmatropic process, but still quite high. The biradical intermediate Int-c (Figure 3) has a C1-C8 distance of $2.983 \AA$ and a C5-C8 distance of $3.090 \AA$ (compared to $2.322 \AA$ and $2.275 \AA$ for the concerted transition state TS-b). Finally, further rotation of the exocyclic radical and ring closure through transition state $\mathbf{T S}-\mathbf{c} 2\left(\Delta G^{\ddagger}=7.3 \mathrm{kcal} / \mathrm{mol}\right)$ generates product $\mathbf{1 0}$.

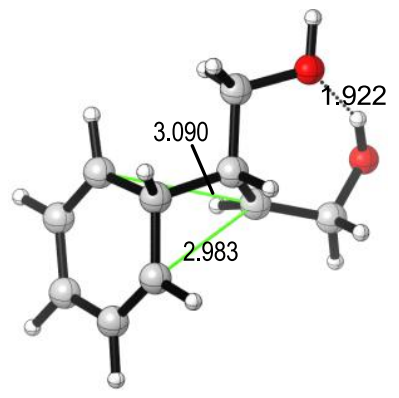

Figure 3. Biradical intermediate (Int-c) in the sigmatropic stepwise process (UM06-2X/6$31+\mathrm{G}(\mathrm{d}, \mathrm{p}))$ for the thermal equilibration of bicyclo[4.2.0]octa-2,4-diene 9 .

Relative Gibbs free energies for all pathways, calculated with three different functionals (B3LYP, M06-2X and B3LYP-D3) and a 6-31+G(d,p) basis set are shown in Table 1. All relative free energies calculated at the M06-2X level of theory are higher than those at the B3LYP level of theory. However, it can be seen that long-range dispersion effects are very small in these systems, as B3LYP and B3LYP-D3 values are almost equal. As expected, the electrocyclic cascade is clearly preferred over both sigmatropic pathways, which have much higher activation barriers at all levels of theory (26.0 versus 59.9 and $44.8 \mathrm{kcal} / \mathrm{mol}$ for the electrocyclic, the concerted sigmatropic and the stepwise sigmatropic pathways, respectively, at 
the M06-2X level of theory). However, the calculations predict that the activation barriers for the sigmatropic process might be overcome at high temperatures. Within the two sigmatropic pathways, the stepwise pathway is shown to be the most plausible at all levels of theory (the activation barrier is approximately $15 \mathrm{kcal} / \mathrm{mol}$ lower than for the concerted pathway).

Broken-symmetry unrestricted methodology was used for both sigmatropic pathways, but all 3 methods led to the restricted solution for the concerted sigmatropic transition state TS-b, suggesting a closed-shell system without triplet character for this pathway, as indicated by expectation values of total spin $\left\langle\mathrm{S}^{2}\right\rangle$ equal to zero (Table 1). The stepwise sigmatropic pathway on the other hand is proposed to go through open-shell transition states and a corresponding biradical intermediate, as shown by the spin contamination $\left(\left\langle S^{2}\right\rangle=0.8335,1.0372\right.$ and 0.7998 for TS-c1, Int-c and TS-c2, respectively, M06-2X/6-31+G(d,p)). 
Table 1. Relative Gibbs free energies $(\mathrm{kcal} / \mathrm{mol})$ of reactants, transition states, intermediates and products for the thermal rearrangement of bicyclo[4.2.0]octa-2,4-diene diol 9, and expectation values of the total spin $\left\langle\mathrm{S}^{2}\right\rangle$ (in parenthesis), calculated at different levels of theory (LOT) with a 6-31+G(d,p) basis set. ${ }^{\text {a }}$

\begin{tabular}{|c|c|c|c|c|c|c|c|c|c|c|c|c|}
\hline & \multirow[b]{3}{*}{ LOT } & \multirow[b]{3}{*}{9} & \multicolumn{5}{|c|}{ ELECTROCYCLIC } & \multicolumn{4}{|c|}{ SIGMATROPIC } & \multirow[b]{3}{*}{10} \\
\hline & & & \multirow[b]{2}{*}{ TS-a1 } & \multirow[b]{2}{*}{ Int-a1 } & \multirow[b]{2}{*}{ TS-a2 } & \multirow[b]{2}{*}{ Int-a2 } & \multirow[b]{2}{*}{ TS-a3 } & \multirow{2}{*}{$\begin{array}{c}\text { Concerted } \\
\text { TS-b }\end{array}$} & \multicolumn{3}{|c|}{ Stepwise } & \\
\hline & & & & & & & & & TS-c1 & Int-c & TS-c2 & \\
\hline \multirow{4}{*}{ Singlet } & B3LYP & 0.0 & 22.7 & 2.4 & 6.5 & 3.2 & 22.9 & $\begin{array}{c}51.0 \\
(0.0000)\end{array}$ & $\begin{array}{c}37.7 \\
(0.7865)\end{array}$ & $\begin{array}{c}32.6 \\
(1.0391)\end{array}$ & $\begin{array}{c}38.7 \\
(0.8110)\end{array}$ & 0.1 \\
\hline & B3LYP-D3 & 0.0 & 22.1 & 1.6 & 6.0 & 2.6 & 22.3 & $\begin{array}{c}51.2 \\
(0.0000)\end{array}$ & $\begin{array}{c}36.9 \\
(0.8455)\end{array}$ & $\begin{array}{c}33.4 \\
(1.0390)\end{array}$ & $\begin{array}{c}38.9 \\
(0.8227)\end{array}$ & 0.1 \\
\hline & M06-2X & 0.0 & 26.0 & 3.4 & 10.0 & 4.5 & 26.1 & $\begin{array}{c}59.9 \\
(0.0000)\end{array}$ & $\begin{array}{c}44.8 \\
(0.8335)\end{array}$ & $\begin{array}{c}39.7 \\
(1.0372)\end{array}$ & $\begin{array}{c}47.0 \\
(0.7998)\end{array}$ & 0.0 \\
\hline & $\begin{array}{l}\text { CASSCF// } \\
\text { UM06-2X }\end{array}$ & 0.0 & 36.8 & 3.8 & 11.1 & 4.5 & 37.1 & 65.8 & 40.6 & 39.4 & 44.8 & 0.0 \\
\hline Triplet & $\begin{array}{l}\text { CASSCF// } \\
\text { UM06-2X }\end{array}$ & 54.7 & 76.0 & 49.6 & 55.0 & 50.0 & 75.9 & 146.1 & 69.7 & 38.8 & 72.2 & 56.6 \\
\hline
\end{tabular}

${ }^{\mathrm{a}}$ Unrestricted methodology for the sigmatropic processes. ${ }^{\mathrm{b}} \mathrm{CASSCF}(6,6) / 6-31+\mathrm{G}(\mathrm{d}, \mathrm{p}) / / \mathrm{UM} 06-2 \mathrm{X} / 6-31+\mathrm{G}(\mathrm{d}, \mathrm{p}) .{ }^{\mathrm{c}}$ Energies relative to singlet reactant 9. 
It should be noted that calculations with both the B3LYP and the B3LYP-D3 level of theory gave rise to an internal instability of the wavefunction for the sigmatropic concerted transition state and analytic frequency calculations are only valid if the wavefunction has no internal instabilities. However, M06-2X calculations gave rise to stable wavefunctions for all pathways under study. Therefore, further calculations were done only with the M06-2X level of theory and the CASSCF calculations in the next subtopic were carried out with M06-2X optimized structures.

\section{CASSCF calculations}

Although several sigmatropic shift studies on pericyclic reactions point out that inexpensive methods such as B3LYP predict activation barriers and energies in excellent agreement with

experimental data, ${ }^{39,65,69-75}$ the biradical intermediate in the stepwise sigmatropic pathway implies the necessity of a multiconfigurational self-consistent field (MCSCF) method, such as the complete active space self-consistent field (CASSCF) method, ${ }^{76}$ which was proven to be valuable for the study of organic reactions. ${ }^{77-79}$

With an initial approximate DMRG calculation in an active space of 66 electrons in 66 ROHF molecular orbitals, which contains all valence electrons, approximate natural orbitals and their occupation numbers were found. Natural orbitals with $0.01<$ NOON $<1.99$ were regarded as essential for the CASSCF calculations, yielding a common active space of 6 electrons in 6 orbitals.

The converged relative Gibbs free $\operatorname{CASSCF}(6,6) / 6-31+\mathrm{G}(\mathrm{d}, \mathrm{p})$ energies of singlet and triplet transition states and intermediates for all pathways under study are shown in Table 1 . The triplet energies are much higher than the singlet energies, except for the biradical intermediate, which has comparable energies for its singlet and triplet forms, indicating that all pathways proceed via 
singlet states. Whereas CASSCF and M06-2X energies are in very good agreement for the sigmatropic pathways, as can be seen by differences of maximum $5.9 \mathrm{kcal} / \mathrm{mol}$, differences of up to $11 \mathrm{kcal} / \mathrm{mol}$ were found for the electrocyclic pathway. Because of these large differences, the clear preference for the electrocyclic pathway over the sigmatropic pathways obtained from DFT calculations (26.0 versus 59.9 and $44.8 \mathrm{~kJ} / \mathrm{mol}$ for the electrocyclic, the concerted sigmatropic and the stepwise sigmatropic pathways, respectively, at the M06-2X level of theory) is not reproduced by the CASSCF calculations (36.8 versus $40.6 \mathrm{~kJ} / \mathrm{mol}$ for the electrocyclic and the stepwise sigmatropic pathways, respectively).

The different energetics resulting from DFT and CASSCF calculations are understandable, since DFT captures dynamic correlation, but not static correlation and CASSCF captures static correlation but not dynamic correlation. Nonetheless, CASSCF indicates a closed shell for the singlet and two radical electrons for the triplet in the electrocyclic pathway (Table 2, further elaborated below), indicating that single Slater determinants are able to describe these structures, hence energetics from the single Kohn-Sham Slater determinant in DFT calculations are deemed reliable. However, if more exotic electronic structures are found, the single Kohn-Sham Slater determinant which lies at the basis of DFT is unable to describe the electronic structure and one needs to resort to CASPT2 for accurate energetics to describe both static and dynamic correlation well. ${ }^{80-81}$ The singlet transition states and intermediate structures of the sigmatropic pathways have more exotic electronic structures (Table 2), indicating the necessity for CASPT2 calculations in order to get accurate energetics. However, CASSCF and CASPT2 energies were shown to be comparable for [1,3] sigmatropic rearrangements of bicyclic and tricyclic vinylcyclobutanes, ${ }^{82}$ which are described by transition states highly similar in nature to the sigmatropic stepwise transition states in the present study, hence the levels of theory employed 
are considered to be sufficient. Moreover, CASSCF and M06-2X energies agree reasonably well for both sigmatropic pathways.

The converged NOON of singlet and triplet transition states and intermediates are shown in Table 2. As expected, the NOON for the first two bonding orbitals are close to 2.0 and those for the last two antibonding orbitals are close to 0.0 for all structures. Furthermore, the NOON for the principal bonding/antibonding pair are close to $1.0 / 1.0$ for all triplet structures. However, while the NOON for the principal bonding/antibonding pair for the singlet structures of the electrocyclic process are close to 2.0/0.0, indicating very little diradical character, they are further off for the sigmatropic processes. More in particular, the concerted sigmatropic transition state has some diradical character $(\mathrm{NOON}$ for the principal bonding/antibonding pair $=1.7 / 0.3)$, the sigmatropic stepwise transition states have even higher diradical character (NOON for the principal bonding/antibonding pair $=1.5 / 0.5)$ and the sigmatropic stepwise intermediate is obviously a pure diradical (NOON for the principal bonding/antibonding pair $=1.0 / 1.0$ ). In summary, CASSCF calculations indicate that all sigmatropic transition states have some diradical character, whereas the sigmatropic stepwise intermediate is a pure diradical. DFT calculations had accurately suggested that the stepwise sigmatropic pathway goes through openshell transition states and a corresponding biradical intermediate; however they had incorrectly suggested the concerted sigmatropic pathway to proceed through a closed-shell transition state. 
Table 2. Natural orbital occupation numbers (NOON) of transition states and intermediates for the thermal rearrangement of bicyclo[4.2.0]octa-2,4-diene diol $9(\operatorname{CASSCF}(6,6) / 6-31+\mathrm{G}(\mathrm{d}, \mathrm{p}) / / \mathrm{UM} 06-2 \mathrm{X} / 6-31+\mathrm{G}(\mathrm{d}, \mathrm{p}))$.

\begin{tabular}{|c|c|c|c|c|c|c|c|c|c|c|c|c|}
\hline \multirow[b]{2}{*}{9} & \multicolumn{6}{|c|}{ Singlet } & \multicolumn{6}{|c|}{ Triplet } \\
\hline & 1.983 & 1.938 & 1.900 & 0.103 & 0.058 & 0.017 & 1.982 & 1.912 & 1.026 & 0.975 & 0.087 & 0.018 \\
\hline TS-a1 & 1.947 & 1.880 & 1.864 & 0.137 & 0.120 & 0.051 & 1.915 & 1.895 & 1.013 & 0.988 & 0.106 & 0.084 \\
\hline Int-a1 & 1.933 & 1.915 & 1.899 & 0.110 & 0.080 & 0.062 & 1.926 & 1.887 & 1.005 & 0.996 & 0.115 & 0.071 \\
\hline TS-a2 & 1.939 & 1.917 & 1.873 & 0.133 & 0.083 & 0.055 & 1.915 & 1.900 & 1.018 & 0.984 & 0.103 & 0.080 \\
\hline Int-a2 & 1.933 & 1.915 & 1.898 & 0.111 & 0.081 & 0.063 & 1.925 & 1.888 & 1.006 & 0.995 & 0.114 & 0.072 \\
\hline TS-a3 & 1.947 & 1.881 & 1.864 & 0.137 & 0.120 & 0.051 & 1.915 & 1.895 & 1.013 & 0.988 & 0.106 & 0.084 \\
\hline TS-b & 1.943 & 1.880 & 1.730 & 0.272 & 0.120 & 0.055 & 1.903 & 1.817 & 1.061 & 0.939 & 0.185 & 0.094 \\
\hline TS-c1 & 1.933 & 1.888 & 1.544 & 0.458 & 0.112 & 0.064 & 1.921 & 1.894 & 1.027 & 0.974 & 0.108 & 0.076 \\
\hline Int-c & 1.931 & 1.885 & 1.001 & 1.000 & 0.116 & 0.067 & 1.922 & 1.894 & 1.028 & 0.974 & 0.108 & 0.076 \\
\hline TS-c2 & 1.934 & 1.890 & 1.536 & 0.467 & 0.110 & 0.064 & 1.931 & 1.884 & 1.006 & 0.995 & 0.117 & 0.067 \\
\hline 10 & 1.983 & 1.938 & 1.900 & 0.103 & 0.058 & 0.017 & 1.981 & 1.912 & 1.025 & 0.977 & 0.087 & 0.019 \\
\hline
\end{tabular}


As a conclusion, the electrocyclic cascade is obviously preferred over both sigmatropic pathways, however, the activation barriers for the stepwise sigmatropic processes might be overcome at high temperatures. Secondly, DFT calculations suggested that the stepwise sigmatropic pathway goes through open-shell transition states and a corresponding biradical intermediate and that the concerted sigmatropic pathway has a closed-shell transition state without triplet character. NOON from CASSCF calculations on the other hand, showed that all sigmatropic transition states have some diradical character and the sigmatropic stepwise intermediate is a pure diradical.

\section{B. Experimental Study}

In order to experimentally investigate the possibility of a [1,5] sigmatropic alkyl shift in a bicyclo[4.2.0] octa-2,4-diene system, the diol derivative 9 was synthesized in two steps from $(E)$ pent-2-en-4-yn-1-ol 11 (Scheme 6). Copper-mediated oxidative Glaser coupling and partial hydrogenation of the resulting symmetrical diyne diol 12 was followed in situ by a cascade of an $8 \pi$ - and a $6 \pi$-electrocyclic ring closures as previously described, ${ }^{2,12}$ giving the $4 \pi$ system 9 as the major product, in reasonable yield. When the reaction was run using deuterium gas $(99.8 \%$ atom D), the expected diol 9--d $\mathbf{4}$ was obtained as a single isotopomer. This deuterium-labeled system was then used to study the thermal rearrangements. 
11

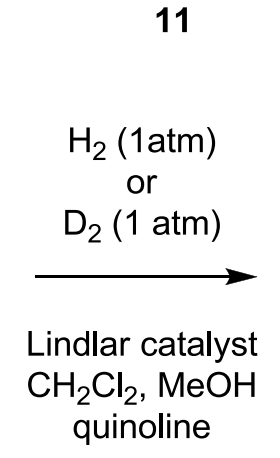

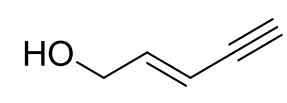

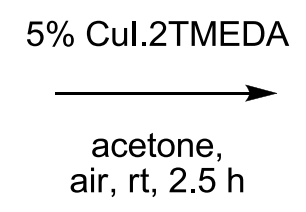

ir, rt, $2.5 \mathrm{~h}$

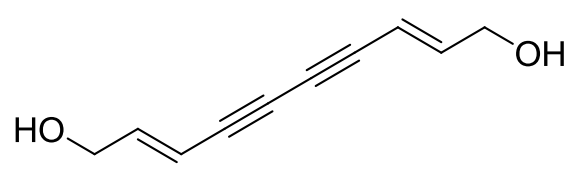

12

(67\%)

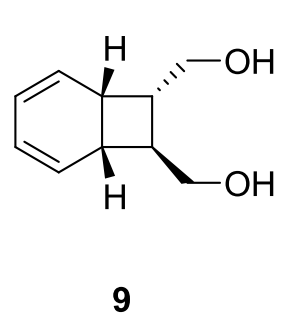

(30-45\%)

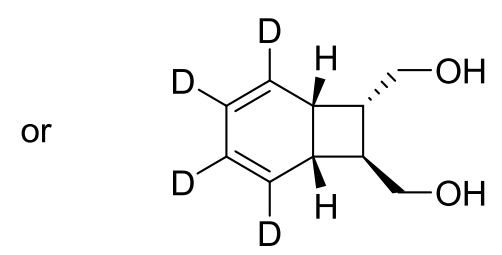

9-d (30-45\%)

Scheme 6. Synthesis of the the bicyclo[4.2.0]octa-2,4-diene diols 9 and 9-d $\mathbf{4}$.

The methyl esters of Endiandric Acids D and E are known to interconvert with a half life of ca. $1.3 \mathrm{~h}$ at $70^{\circ} \mathrm{C}$ in toluene. Thus, a similar equilibrium is expected to exist in the simpler diol $\mathbf{9}$, although the interconverting products are identical in this case. However, for the deuteriumlabeled diol 9-d $\mathbf{4}$, this equilibrium would be unnoticed only if the rearrangement followed exclusively the electrocyclic ring opening pathway to the $6 \pi$ cyclooctatriene (COT) valence tautomer, which can then ring flip and close again, whereas the alternative single step pathway via a [1,5] sigmatropic alkyl shift or walk rearrangement would result in extensive scrambling of the deuterium labels over the carbons of the six membered ring (Scheme 5, b). The expected isotopomers of diol 9-d $\mathbf{4}$ should be detected easily by the appearance of the diagnostic olefinic resonances in the proton NMR spectrum. However, when a solution of the diol 9-d $\mathbf{4}$ in toluene was heated at $110^{\circ} \mathrm{C}$ for $1 \mathrm{~h}$, the starting material was recovered unchanged, by NMR analysis. Consequently, the thermal equilibrium previously described by Nicolaou and Black for the Endiandric Acids does not constitute a walk rearrangement, as previously demonstrated also by computational results. 
When dilute solutions of $\mathbf{9 - d _ { 4 }}$ were heated at temperatures between 170 and $195^{\circ} \mathrm{C}$, olefinic resonances did appear in the proton NMR spectra, which were superimposable with those observed for the non-labeled diol 9 both in $\mathrm{CDCl}_{3}$ and DMSO-d $\mathrm{d}_{6}$. However, assignment to any of the six possible isotopomers was not possible via 1D or 2D NMR experiments. Rigorous chromatographic purification of the reaction mixture obtained after heating for 2 hours at 190$195^{\circ} \mathrm{C}$ in acetonitrile (sealed tube), gave the unchanged diol 9- $\mathbf{d}_{\mathbf{4}}$ as a single isotopomer in about $30 \%$ yield. The observed olefinic resonances could therefore not be explained as D-scrambled products, but must arise from other thermal reaction products. A similar complex mixture of products was obtained when the non-labeled diol 9 was subjected to the same conditions, but none of the constituents could be fully identified. Finally, heating diols $\mathbf{9}$ and $\mathbf{9 - d _ { 4 }}$ at even higher temperatures (up to $230^{\circ} \mathrm{C}$ ) in ethylene glycol (sealed tube) gave a very fast $(<10 \mathrm{~min})$ and complete consumption of the starting material, returning a rather complex and inseparable mixture of products. In contrast to most bicyclo[4.1.0]hepta-2,4-diene (norcaradiene) systems, the bicyclo[4.2.0]octa-2,4-diene system appears to have limited thermal stability. Furthermore, partial analysis by 2D NMR experiments seems to implicate the completely ring-opened acyclic tetraene valence tautomer as the parent structure for most of the observed thermal products, a reaction pathway, which is not available for the norcaradiene systems.

\section{Thermal Equilibration of Bicyclo[4.2.0]octa-2,4-diene 7 and comparison with literature}

The same pathways were computationally explored for the parent bicyclo[4.2.0]octa-2,4-diene compound 7 (Figure 4) in order to compare the thermal equilibration of this unsubstituted bicyclo[4.2.0] octa-2,4-diene with the norcaradiene system reported in literature. As mentioned previously, the M06-2X/6-31+G(d,p) level of theory was used since M06-2X calculations on the thermal equilibration of bicyclo[4.2.0]octa-2,4-diene 9 gave rise to stable wavefunctions for all 
pathways under study, whereas calculations with both the B3LYP and the B3LYP-D3 level of theory gave rise to an internal instability of the wavefunction for the sigmatropic concerted transition state. However, all three levels of theory led to exactly the same trends.

The free energy profiles for all pathways of the thermal equilibration of bicyclo[4.2.0]octa-2,4diene 7 are shown in Figure 4. These look fairly similar to the free energy profiles for bicyclo[4.2.0]octa-2,4-diene 9, again suggesting a clear preference for the electrocyclic cascade. However, while the bicyclo[4.2.0]octa-2,4-diene 9 was found to be more stable than its contorted cyclooctatriene intermediate, as anticipated in the introduction, this is not true for the bicyclo[4.2.0]octa-2,4-diene 7, which was found to be as stable as its contorted cyclooctatriene intermediate, as was recently reported by Houk. ${ }^{83}$ Consequently, the sigmatropic pathways are shown to be less likely for bicyclo[4.2.0]octa-2,4-diene 7 when compared to the bicyclo[4.2.0] octa-2,4-diene 9. More importantly, no stable singlet intermediate could be located for the unsubstituted bicyclo[4.2.0]octa-2,4-diene 7, whereas a singlet biradical intermediate was found for bicyclo[4.2.0] octa-2,4-diene 9, which can be attributed to the difference in stability for primary and secondary radicals. Hence, the stepwise sigmatropic pathway is not plausible for the parent bicyclo[4.2.0]octa-2,4-diene compound 7 and the concerted sigmatropic pathway is highly $\operatorname{activated}\left(\Delta G^{\ddagger}=57.1 \mathrm{kcal} / \mathrm{mol}\right)$. 


\section{TS-b'}

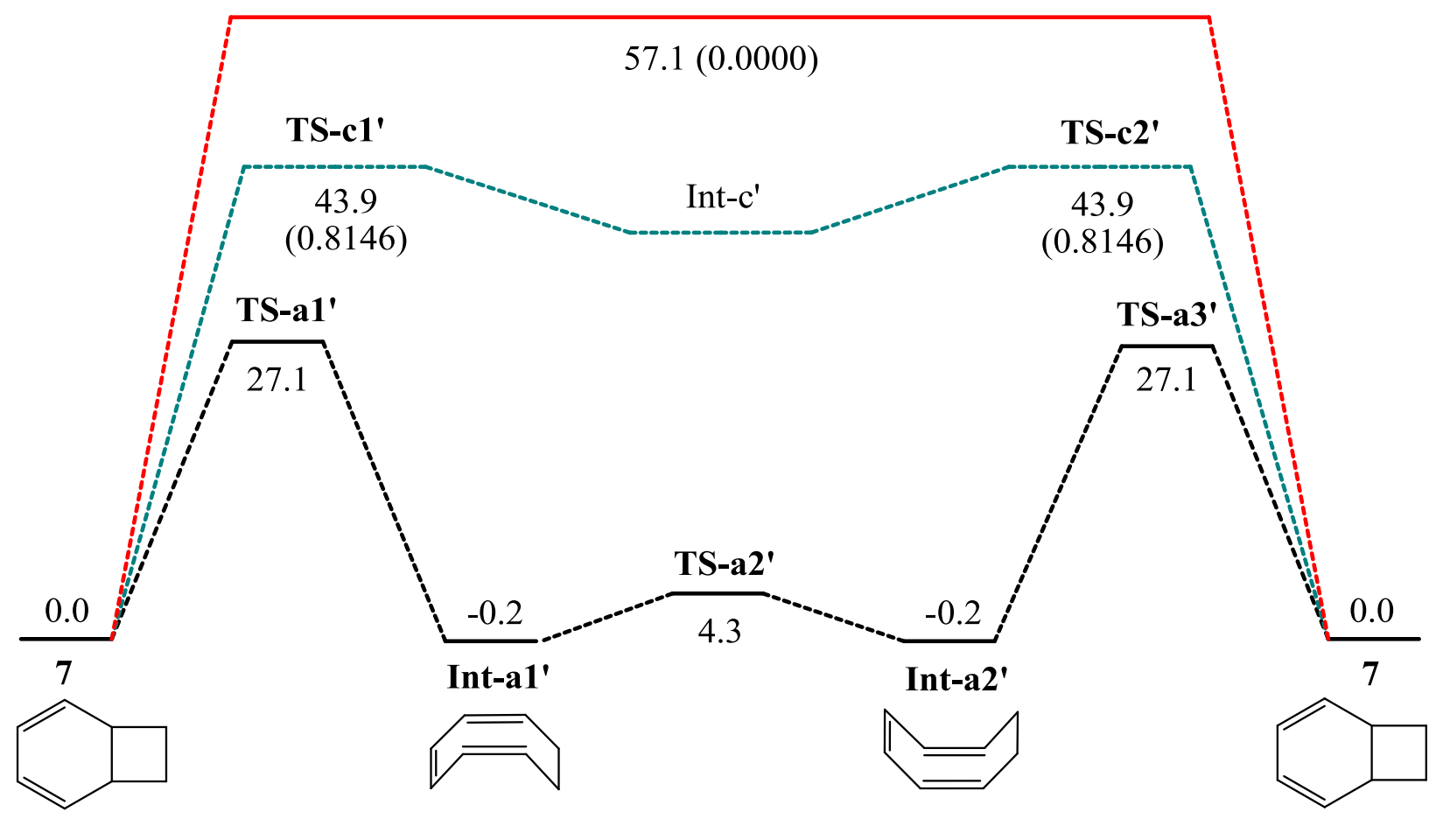

Electrocyclic

Sigmatropic concerted

Sigmatropic stepwise

Figure 4. Free energy profile for the electrocyclic (M06-2X/6-31+G(d,p)) and sigmatropic pathways (UM06-2X/6-31+G(d,p)) and expectation values of the total spin $\left\langle\mathrm{S}^{2}\right\rangle$ (in parenthesis) in the thermal equilibration of bicyclo[4.2.0]octa-2,4-diene 7., ${ }^{\mathrm{a} b}$ All energies in $\mathrm{kcal} / \mathrm{mol}$.

${ }^{\mathrm{a}}$ For the stepwise sigmatropic pathway, no stable intermediate could be located and IRC paths lead to an unstable intermediate that disintegrated. ${ }^{\mathrm{b}} \mathrm{UM} 06-2 \mathrm{X}$ means the unrestricted version of M06-2X.

The Gibbs free activation barrier for the electrocyclic route found here is very close to the ones reported earlier by Huisgen ${ }^{42 \mathrm{~b}}$ and recently by $\operatorname{Houk}^{83}\left(\Delta G^{\ddagger}=27.1 \pm 0.2 \mathrm{kcal} / \mathrm{mol}\right)$. Furthermore, although the parent bicyclo[4.2.0]octa-2,4-diene compound 7 cannot undergo the stepwise sigmatropic route, appropriate substituents can favor this pathway, as demonstrated in the previous section for bicyclo[4.2.0]octa-2,4-diene 9. The calculated barriers for the sigmatropic bond cleavage of bicyclo[4.2.0]octa-2,4-diene $9\left(\Delta G^{\ddagger}=44.8\right.$ and $\left.47.0 \mathrm{kcal} / \mathrm{mol}\right)$ are within the range of experimental and predicted activation barriers for [1,5] alkyl shifts in 
bicyclo[4.1.0]hepta-2,4-dienes, which range from 35 to $45 \mathrm{kcal} / \mathrm{mol} .^{38-39}$

As a conclusion, even though the electrocyclic cascade is more plausible, comparable barriers for the [1,5] alkyl shifts of bicyclo[4.2.0]octa-2,4-dienes and bicyclo[4.1.0]hepta-2,4-dienes, strongly suggest that the sigmatropic stepwise pathway is feasible at higher temperatures for appropriately substituted compounds.

\section{Thermal Equilibration of Endiandric Acid Methyl Esters D/E (1/2)}

Finally, the three pathways were computationally explored for the thermal equilibration of endiandric acid methyl esters D/E (1/2). The free energy profiles shown in Figure 5, reveal relative Gibbs free energies that are only slightly higher than those for the thermal equilibration of the diol derivate 9 for all pathways and expectation values of total spin $\left\langle S^{2}\right\rangle$ that are comparable to those for the diol derivate 9, suggesting a clear preference for the electrocyclic cascade. The sigmatropic stepwise pathway might be feasible at high temperatures. The energetically favorable electrocyclic pathway along with optimized transition state and intermediate geometries is shown in Figure 6. A thorough conformational search was done on the phenyl pentadienyl group and the methyl ester group of methyl ester $\mathbf{1}$ and the most stable conformer is shown in Figure 6. All other transition states and intermediates originated from this conformation. Endiandric acid methyl esters $\mathbf{1 / 2}$ were found to be yet more stable with respect to their contorted cyclooctatriene (COT) intermediate, compared to the diol derivate $\mathbf{9}$, which is in favor of the sigmatropic pathways. Moreover, substituents made it possible to locate a stable singlet intermediate for the stepwise sigmatropic pathway, whereas no stable intermediate could be located for the unsubstituted bicyclo[4.2.0]octa-2,4-diene. Therefore, the stepwise sigmatropic pathway may be plausible at higher temperatures for the diol derivate $\mathbf{9}$ and endiandric acid methyl esters 1/2, but not for the parent bicyclo[4.2.0]octa-2,4-diene compound $\mathbf{7}$. 
Expectation values of total spin $\left\langle S^{2}\right\rangle$ from DFT calculations indicate that the stepwise sigmatropic pathway goes through open-shell transition states and a corresponding biradical intermediate, whereas the concerted sigmatropic pathway has a closed-shell transition state without triplet character. However, NOON from CASSCF calculations on the diol derivate 9 showed that all sigmatropic transition states have some diradical character, while the sigmatropic stepwise intermediate is a pure diradical. Since the relative Gibbs free energies for the thermal equilibration of methyl esters $\mathbf{1 / 2}$ are comparable to those for the thermal equilibration of the diol derivate 9 for all pathways, and CASSCF and M06-2X energies are in very good agreement for the sigmatropic transition states, and because of the large size of the system, CASSCF calculations were not performed on the sigmatropic pathways of the thermal equilibration of methyl esters $\mathbf{1 / 2}$. However, these are considered to be comparable to the calculations on the diol derivate 9, indicating that all pathways proceed via singlet states and the sigmatropic pathways have diradical character, but that M06-2X energies agree reasonably well. 


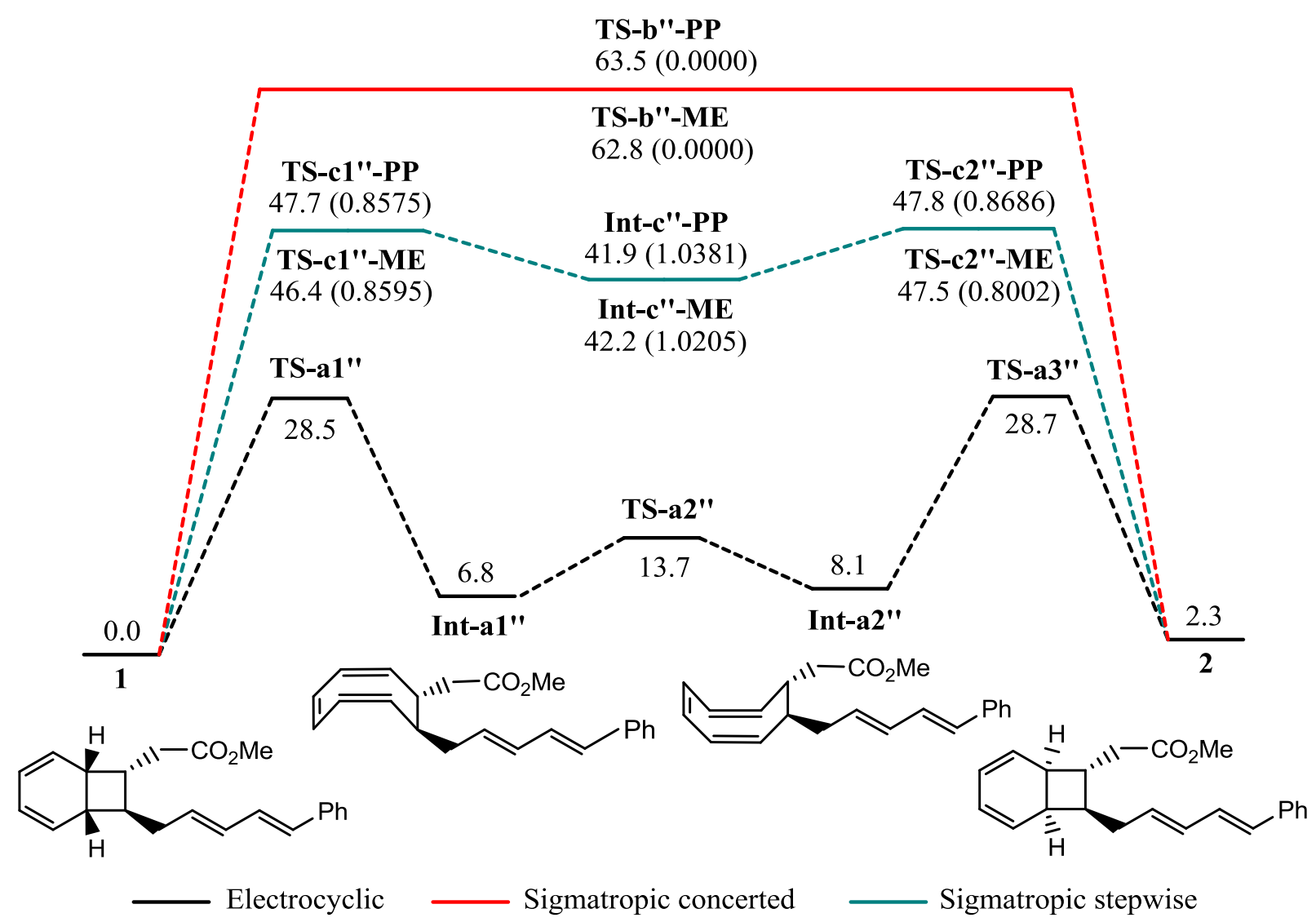

Figure 5. Free energy profile for the electrocyclic $(M 06-2 X / 6-31+G(d, p))$ and sigmatropic pathways (UM06-2X/6-31+G(d,p)) and expectation values of the total spin $\left\langle\mathrm{S}^{2}\right\rangle$ (in parenthesis) in the thermal equilibration of endiandric acid methyl esters $\mathrm{D} / \mathrm{E}(\mathbf{1} / \mathbf{2}){ }^{\mathrm{a}}$ All energies in $\mathrm{kcal} / \mathrm{mol}$. ${ }^{a}$ For the sigmatopic pathways, PP indicates the breaking of the $\mathrm{C}-\mathrm{C}$ bond close to the phenyl pentadienyl group and ME indicates the breaking of the C-C bond close to the methyl ester group. 


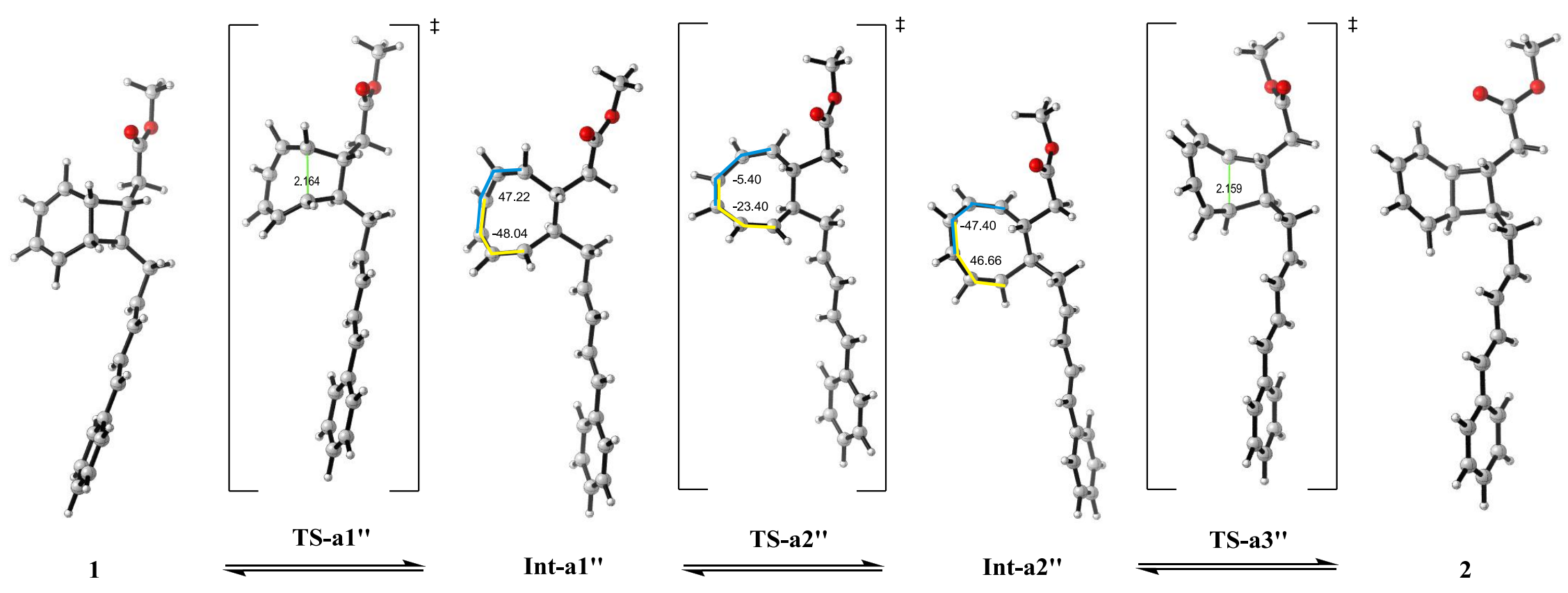

Figure 6. Electrocyclic cascade (M06-2X/6-31+G(d,p)) for the thermal equilibration of endiandric acid methyl esters D/E (1/2).

Some critical distances (green, $\AA$ ) and dihedral angles (yellow and blue, in degrees) are shown. 


\section{CONCLUSION}

The mechanism of thermal equilibration between endiandric acid methyl esters D/E in particular and more generally the possibility of [1,5] sigmatropic alkyl shifts (walk rearrangements) in bicyclo[4.2.0]octa-2,4-diene systems at high temperatures have been explored in a combined computational and experimental study, pointing to the following conclusions: a) An electrocyclic cascade is clearly preferred over both sigmatropic pathways. The calculated free energy barriers for this route, which was previously proposed by Nicolaou, are shown to be very close to the one for bicyclo[4.2.0]octa-2,4-diene reported by Huisgen. (b) The activation barriers for the sigmatropic processes might be overcome at high temperatures. (c) The sigmatropic stepwise pathway is shown to be significantly lower in energy than the sigmatropic concerted pathway and calculated barriers for this alkyl group shift were shown to be comparable with the reaction barriers for the bicyclo[4.1.0]hepta-2,4-diene (norcaradiene) walk rearrangement. Nevertheless, this stepwise pathway is only feasible for appropriately substituted compounds. (d) DFT calculations suggested that the stepwise sigmatropic pathway goes through open-shell transition states and a corresponding biradical intermediate, whereas the concerted sigmatropic pathway has a closed-shell transition state without triplet character. However, CASSCF calculations showed that all sigmatropic transition states have some diradical character, while the sigmatropic stepwise intermediate is a pure diradical.

Experimental NMR analysis on the thermal rearrangement of the deuterium labeled diol (9-d 4 ), for which the electrocyclic and sigmatropic rearrangements would lead to different interconverting isotopomeric products, showed that in this model system, [1,5] sigmatropic alkyl shifts do not occur with a significant reaction rate at temperatures up to $195^{\circ} \mathrm{C}$. Higher temperatures could not be explored because of the limited thermal stability of this bicyclic 
system. Our results indicate that, although [1,5] sigmatropic shifts should be energetically comparable processes both in bicyclo[4.2.0]octa-2,4-diene and bicyclo[4.1.0]hepta-2,4-diene compounds, they have so far only been observed in the latter.

\section{EXPERIMENTAL SECTION}

General methods, materials, synthetic procedures and spectral data for compounds $\mathbf{9 ,} \mathbf{1 1}$ and $\mathbf{1 2}$ are reported in the Supporting Information.

[2,3,4,5- $\left.{ }^{2} \mathrm{H}_{4}\right]-(8-H y d r o x y m e t h y l-b i c y c l o[4.2 .0]$ octa-2,4-dien-7-yl)-methanol 9-d 4 . The general procedure for the synthesis of bicyclic diol 9 was followed (see Supporting Information), but deuterium gas (99.8\% atom D) was used instead of hydrogen gas. Starting from $50 \mathrm{mg}$ of diol $\mathbf{1 2}$ (0.295 mmol), chromatography as described for compound $\mathbf{9}$ gave the deuterium labeled bicyclic diol 9-d 4 (18.0 mg, 34\%) as a clear viscous oil. IR $v_{\max } 3336(\mathrm{~s}), 2922(\mathrm{~s}), 1462,1376,1025 ;{ }^{1} \mathrm{H}$ NMR (300 MHz, $\left.\mathrm{CDCl}_{3}\right): \delta$ 2.67-2.74 (3H, band, 3 x CH), 3.14-3.19 (1H, m, =CD-CH), 3.45$3.51(1 \mathrm{H}, \mathrm{m}, \mathrm{CH} \mathrm{HOH}), 3.76(1 \mathrm{H}, \mathrm{dd}, J=10.2$ and $3.6 \mathrm{~Hz}, \mathrm{CHHOH}), 3.79-3.85(2 \mathrm{H}, \mathrm{m}$, $\left.\mathrm{CH}_{2} \mathrm{OH}\right) ;{ }^{13} \mathrm{C}$ NMR $\left(75 \mathrm{MHz}, \mathrm{CDCl}_{3}\right): \delta 32.5(\mathrm{CH}), 33.0(\mathrm{CH}), 51.1(\mathrm{CH}), 52.5(\mathrm{CH}), 62.8$ $\left(\mathrm{CH}_{2}\right), 65.5\left(\mathrm{CH}_{2}\right) ; \mathrm{MS}(\mathrm{ESI}) \mathrm{m} / \mathrm{z} 171.1\left(\mathrm{MH}^{+}, 58\right), 153.1\left(\mathrm{MH}^{+}-\mathrm{H}_{2} \mathrm{O}, 100\right)$; HRMS (ESI) calcd. for $\mathrm{C}_{10} \mathrm{D}_{4} \mathrm{H}_{11} \mathrm{O}_{2}\left(\mathrm{~m} / \mathrm{z} \mathrm{M}+\mathrm{H}^{+}\right)$: 171.1318 , found: 171.1323 .

Thermal equilibration experiments. A solution of the diol $\mathbf{9}$ or the diol 9-d 4 ( 2 to $5 \mathrm{mg}$ per run) in acetonitrile $(2.0 \mathrm{ml})$ was neutralized with $\sim 1 \mathrm{mg}$ of sodium bicarbonate and purged with argon. The solution was then stirred in a closed reaction vessel under microwave heating (CEM Discover). The reaction temperature and vessel pressure were monitored by external surface sensors. Reactions in acetonitrile were maintained at temperatures between either $170-175{ }^{\circ} \mathrm{C}$ or 
190-195 ${ }^{\circ} \mathrm{C}$ for 1-6 hours (which was the highest temperature that could be achieved in this solvent $\left(\mathrm{p}_{\max }=17.0\right.$ bar $)$ ). Reactions were monitored by TLC and NMR, and the reaction mixtures were increasingly complex with reaction temperature and time. The starting materials were isolated unchanged from the reaction mixtures by careful chromatography over silica, eluting with $2 \%$ methanol in chloroform. The obtained products $(0.5-2.5 \mathrm{mg}, 25-50 \%)$ showed

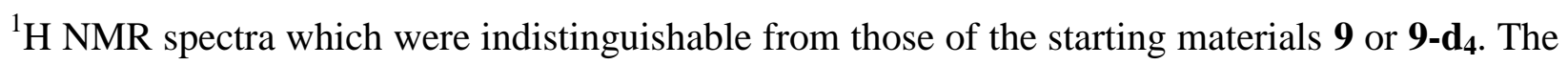
same experiments performed in ethylene glycol, which allows reaction temperatures higher than $200{ }^{\circ} \mathrm{C}$, gave similar results. However, no trace of starting material remained after heating to 230 ${ }^{\circ} \mathrm{C}$ (20 min) in these experiments, as judged by TLC and NMR.

Supporting Information Available: Cartesian coordinates and energy of M06-2X/6-31+G(d,p) optimized geometries, imaginary and low frequencies of transition states. Full references of Gaussian 09 (reference 59). Synthetic procedures and spectral data for compounds 11, 12 and 9. NMR spectra for compounds 11, 12,9 and 9-d 4 . This material is available free of charge via the Internet at http://pubs.acs.org.

\section{ACKNOWLEDGEMENTS}

The Fund for Scientific Research Flanders (FWO) and the Research Board of Ghent University are acknowledged for financial support. L. H. thanks the Spanish MICIIN (MAT2011-29174C02-02) for financial support. Computational resources and services used in this work were provided by Ghent University.

\section{REFERENCES AND NOTES}

(1) Bandaranayake, W. M.; Banfield, J. E.; Black, D. S. C.; Fallon, G. D.; Gatehouse, B. M. Chem. Commun. 1980, 162. 
(2) Bandaranayake, W. M.; Banfield, J. E.; Black, D. S. C. Chem. Commun. 1980, 902.

(3) Azmi, M. N.; Gény, C.; Leverrier, A.; Litaudon, M.; Dumontet, V.; Birlirakis, N.; Guéritte, F.; Leong, K. H.; Halim, S. N. A.; K.; Awang, K. Molecules 2014, 19, 1732.

(4) Chouna, J. R.; Nkeng-Efouet, P. A.; Lenta, B. N.; Devkota, K. P.; Neumann, B.; Stammler, H.-G.; Kimbu, S. F.; Sewald, N. Phytochemistry 2009, 70, 684.

(5) Chouna, J. R.; Nkeng-Efouet, P. A.; Lenta, B. N.; Wansi, J. D.; Kimbu, S. F.; Sewald, Phytochem. Lett. 2010, 3, 13.

(6) Talontsi, F. M.; Lamshöft, M.; Bauer, J. O.; Razakarivony, A. A.; Andriamihaja, B.; Strohmann, C.; Spiteller, M. J. Nat. Prod. 2013, 76, 97.

(7) Yang, P.-S.; Cheng, M.-J.; Peng, C.-F.; Chen, J.-J.; Chen, I.-S. J. Nat. Prod. 2009, $72,53$.

(8) Williams, R. B.; Martin, S. M.; Hu, J.-F.; Norman, V. L.; Goering, M. G.; Loss, S.; O’Neil-Johnson, M.; Eldridge, G. R.; Starks, C. M. J. Nat. Prod. 2012, 75, 1319.

(8) Nicolaou, K. C.; Petasis, N. A.; Zipkin, R. E.; Uenishi, J. J. Am. Chem. Soc. 1982, 104,5555 .

(9) Nicolaou, K. C.; Petasis, N. A.; Uenishi, J.; Zipkin, R. E. J. Am. Chem. Soc. 1982, $104,5557$.

(10) Nicolaou, K. C.; Zipkin, R. E.; Petasis, N. A. J. Am. Chem. Soc. 1982, 104, 5558.

(11) Nicolaou, K. C.; Petasis, N. A.; Zipkin, R. E. J. Am. Chem. Soc. 1982, 104, 5560.

(12) Nicolaou, K. C.; Chen, J. S. Chem. Soc. Rev. 2009, 38, 2993. 
(13) A notable exception is the well known [1,5] hydrogen shift of cyclopentadienes, which is a fast reaction at room temperature, for computational studies see: Hess, B. A.; Baldwin, J. E. J. Org. Chem. 2002, 67, 6025.

(14) Saettel, N. J.; Wiest, O. J. Org. Chem.2000, 65, 2331.

(15) Alabugin, I. V.; Manoharan, M.; Breiner, B.; Lewis, F. D. J. Am. Chem. Soc. 2003, 125, 9329, and references therein.

(16) Tantillo, D. J.; Lee, J. K. Annu. Rep. Prog. Chem., Sect. B: Org. Chem. 2007, 103, 272, and references therein.

(17) Beaudry, C. M.; Malerich, J. P.; Trauner, D. Chem. Rev. 2005, 105, 4757.

(18) Houk, K. N.; Gonzalez, J.; Li, Y. Acc. Chem. Res. 1995, 28, 81.

(19) Houk, K. N.; Li, Y.; Evanseck, J. D. Angew. Chem., Int. Ed. Engl. 1992, 31, 682.

(20) Woodward, R. B.; Hoffmann, R. J. Am. Chem. Soc. 1965, 87, 2511.

(21) Hoffmann, R.; Woodward, R. B. Acc. Chem. Res. 1968, 1, 17.

(22) Woodward, R. B.; Hoffmann, R. Angew. Chem., Int. Ed. Engl. 1969, 8, 781.

(23) Woodward, R. B.; Hoffmann, R. J. Am. Chem. Soc. 1965, 87, 395.

(24) Newman-Evans, R. H.; Simon, R. J.; Carpenter, B. K. J. Org. Chem.1990, 55, 695.

(25) Carpenter, B. K. Angew. Chem., Int. Ed. 1998, 37, 3340.

(26) Reyes, M. B.; Lobbovsky, E. B.; Carpenter, B. K. J. Am. Chem. Soc. 2002, 124, 61 , and references therein.

(27) Carpenter, B. K. J. Am. Chem. Soc. 1995, 117, 6336.

(28) Boersma, M. A. M.; De Haan, J. W.; Kloosterziel, H.; Van de Ven, L. J. M. J. Chem. Soc., Chem. Commun. 1970, 1168. 
(29) Jensen, F. J. Am. Chem. Soc. 1989, 111, 4643.

(30) Klärner, F. G. Top. Stereochem. 1984, 15, 1.

(31) Klärner, F. G. In Topics in Stereochemistry; John Wiley \& Sons, Inc.: 2007, p 1.

(32) Bulo, R. E.; Jansen, H.; Ehlers, A. W.; de Kanter, F. J. J.; Schakel, M.; Lutz, M.; Spek, A. L.; Lammertsma, K. Angew. Chem., Int. Ed. 2004, 43, 714.

(33) Bulo, R. E.; Allaart, F.; Ehlers, A. W.; de Kanter, F. J. J.; Schakel, M.; Lutz, M.; Spek, A. L.; Lammertsma, K. J. Am. Chem. Soc. 2006, 128, 12169.

(34) Jensen, F. J. Am. Chem. Soc. 1989, 111, 4643.

(35) Klärner, F. G.; Wette, M. Chem. Ber. 1978, 111, 282.

(36) Berson, J. A.; Willcott, M. R. J. Am. Chem. Soc. 1966, 88, 2494.

(37) Reyes, M. B.; Lobkovsky, E. B.; Carpenter, B. K. J. Am. Chem. Soc. 2002, 124, 641.

(38) Kless, A.; Nendel, M.; Wilsey, S.; Houk, K. N. J. Am. Chem. Soc. 1999, 121, 4524 , and references therein.

(39) Jarzecki, A. A.; Gajewski, J.; Davidson, E. R. J. Am. Chem. Soc. 1999, 121, 6928.

(40) Klärner, F. G.; Brassel, B. J. Am. Chem. Soc. 1980, 102, 2469.

(41) Baldwin, J. E.; Broline, B. M. J. Am. Chem. Soc. 1982, 104, 2857.

(42) This equilibrium is mainly governed by substitution at C7 and C8 positions, see: a) Huisgen, R.; Dahmen, A.; Huber, H. J. Am. Chem. Soc. 1967, 89, 7130; b) Huisgen, R.; Boche, G.; Dahmen, A.; Hechtl, W. Tetrahedron Lett. 1968, 5215; c) Fry, A. J. Tetrahedron 2008, 64, 2101.

(43) Leber, P. A.; Baldwin, J. E. Acc. Chem. Res. 2002, 35, 279.

(44) Baldwin, J. E. Chem. Rev. 2003, 103, 1197. 
(45) Baldwin, J. E.; Leber, P. A. Org. Biomol. Chem. 2008, 6, 36.

(46) Hudlicky, T.; Reed, J. Angew. Chem., Int. Ed. 2010, 49, 4864.

(47) Bulo, R. E.; Ehlers, A. W.; Grimme, S.; Lammertsma, K. J. Am. Chem. Soc. 2002, 124, 13903.

(48) Hehre, W. J.; Ditchfield, R.; Pople, J. A. J. Chem. Phys. 1972, 56, 2257.

(49) Krishnan, R.; Binkley, J. S.; Seeger, R.; Pople, J. A. J. Chem. Phys. 1980, 72, 650.

(50) Becke, A. D. J. Chem. Phys. 1993, 98, 5648.

(51) Lee, C.; Yang, W.; Parr, R. G. Phys. Rev. B 1988, 37, 785.

(52) Zhao, Y.; Truhlar, D. G. Acc. Chem. Res. 2008, 41, 157.

(53) Zhao, Y.; Truhlar, D. Theor. Chem. Acc. 2008, 120, 215.

(54) Grimme, S.; Antony, J.; Ehrlich, S.; Krieg, S. J. Chem. Phys. 2010, 132, 154104.

(55) Fukui, K. Acc. Chem. Res. 1981, 14, 363.

(56) Hratchian, H. P.; Schlegel, H. B.; Clifford, E. D.; Gernot, F.; Kwang, S. K.; Gustavo, E. S. In Theory and Applications of Computational Chemistry; Elsevier: Amsterdam, 2005, p 195.

(57) Seeger, R.; Pople, J. A. J. Chem. Phys. 1977, 66, 3045.

(58) Bauernschmitt, R.; Ahlrichs, R. J. Chem. Phys 1996, 104, 9047. 
(59) Gaussian 09, Revision A.02, M. J. Frisch et al, Gaussian, Inc., Wallingford CT, 2009.

(60) White, S. R.; Martin, R. L. J. Chem. Phys. 1999, 110, 4127.

(61) Chan, G. K.-L.; Head-Gordon, M. J. Chem. Phys. 2002, 116, 4462.

(62) Wouters, S.; Bogaerts, T.; Van Der Voort, P.; Van Speybroeck V.; Van Neck, D. J. Chem. Phys. 2014, 140, 241103.

(63) Wouters, S.; Poelmans, W.; Ayers, P. W.; Van Neck, D. Comput. Phys. Commun. 2014, 185, 1501.

(64) Wouters, S.; Van Neck, D. Eur. Phys. J. D 2014, 68, 272.

(65) Leach, A. G.; Catak, S.; Houk, K. N. Chem. -Eur. J. 2002, 8, 1290.

(66) Wiest, O.; Montiel, D. C.; Houk, K. N. J. Phys. Chem. A 1997, 101, 8378.

(67) Hrovat, D. A.; Borden, W. T. J. Am. Chem. Soc. 2001, 123, 4069.

(68) Doering, W. v. E.; Ekmanis, J. L.; Belfield, K. D.; Klärner, F. G.; Krawczyk, B. J. Am. Chem. Soc. 2001, 123, 5532.

(69) Guner, V.; Khuong, K. S.; Leach, A. G.; Lee, P. S.; Bartberger, M. D.; Houk, K. N. J. Phys. Chem. A 2003, 107, 11445.

(70) Rodríguez-Otero, J.; Cabaleiro-Lago, E. M.; Peña-Gallego, Á. Tetrahedron 2007, 63, 2191.

(71) Jursic, B. S. Comp. Theor. Chem. 1995, 358, 139.

(72) Jursic, B.; Zdravkovski, Z. Perkin Trans. 2 1995, 1223. 
(73) Goldstein, E.; Beno, B.; Houk, K. N. J. Am. Chem. Soc. 1996, 118, 6036, and references therein.

(74) Beno, B. R.; Wilsey, S.; Houk, K. N. J. Am. Chem. Soc. 1999, 121, 4816.

(75) Houk, K. N.; Beno, B. R.; Nendel, M.; Black, K.; Yoo, H. Y.; Wilsey, S.; Lee, J. K. Comp. Theor. Chem. 1997, 398-399, 169.

(76) Björn, O. R. In Advances in Chemical Physics; Lawley, K. P., Ed. 2007, p 399.

(77) Robb, M. A.; Bernardi, F. New Theoretical Concepts for Understanding Organic Reactions.; Eds: J. Bertran and I. G. Czismadia: Kluwer: Dordrecht, 1989.

(78) Bernardi, F.; Olivucci, M.; McDouall, J. J. W.; Robb, M. A. J. Chem. Phys. 1988, $89,6365$.

(79) Bachrach, S. M. Computational Organic Chemistry; Wiley-Interscience: New Jersey, 2007.

(80) Andersson, K.; Malmqvist, P. A.; Roos, B. O.; Sadlej, A. J.; Wolinski, K. J. Phys. Chem. 1990, 94, 5483.

(81) Andersson, K.; Malmqvist, P.-A.; Roos, B. O. J. Chem. Phys. 1992, 96, 1218.

(82) Tao, H.-R.; Fang, D.-C. Theor. Chem. Acc. 2008, 121, 91.

(83) Patel, A.; Houk K. N. J. Org. Chem. 2014, http://dx.doi.org/10.1021/jo5015728 


\section{Possibility of [1,5] Sigmatropic Shifts in Bicyclo[4.2.0]octa-2,4-dienes}

2 Hannelore Goossens, ${ }^{\dagger}$ Johan M. Winne, ${ }^{\ddagger}$ Sebastian Wouters, ${ }^{\dagger}$ Laura Hermosilla, ${ }^{\S}$ Pierre J. De Clercq, ${ }^{\ddagger}$ ${ }_{3}$ Michel Waroquier, ${ }^{\dagger}$ Veronique Van Speybroeck, ${ }^{\dagger}$ and Saron Catak ${ }^{*},, \|$

$4^{\dagger}$ Center for Molecular Modeling, Ghent University, Technologiepark 903, 9052 Zwijnaarde, Belgium

5 Department of Organic and Macromolecular Chemistry, Ghent University, Krijgslaan 281/S4, 9000 Ghent, Belgium

$6{ }^{\S}$ Departamento de Química Física Aplicada, Universidad Autónoma de Madrid, 28049 Madrid, Spain

7 "Department of Chemistry, Bogazici University, 34342, Bebek, Istanbul, Turkey

\section{S Supporting Information}

9 ABSTRACT: The thermal equilibration of the methyl esters 10 of endiandric acids $\mathrm{D}$ and $\mathrm{E}$ was subject to a computational 11 study. An electrocyclic pathway via an electrocyclic ring 12 opening followed by a ring flip and a subsequent electro13 cyclization proposed by Nicolaou [Nicolaou, K. C.; Chen, J. S. 14 Chem. Soc. Rev. 2009, 38, 2993], was computationally explored.

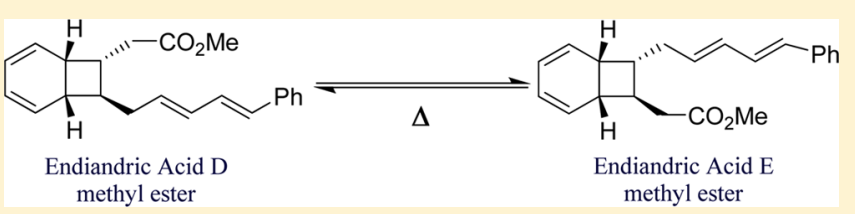

15 The free-energy barrier for this electrocyclic route was shown to be very close to the bicyclo[4.2.0] octa-2,4-diene reported by 16 Huisgen [Huisgen, R.; Boche, G.; Dahmen, A.; Hechtl, W. Tetrahedron Lett. 1968, 5215]. Furthermore, the possibility of a [1,5] 17 sigmatropic alkyl group shift of bicyclo[4.2.0] octa-2,4-diene systems at high temperatures was explored in a combined 18 computational and experimental study. Calculated reaction barriers for an open-shell singlet biradical-mediated stepwise [1,5] 19 sigmatropic alkyl group shift were shown to be comparable with the reaction barriers for the bicyclo[4.1.0]hepta-2,4-diene 20 (norcaradiene) walk rearrangement. However, the stepwise sigmatropic pathway is suggested to only be feasible for appropriately 21 substituted compounds. Experiments conducted on a deuterated analogous diol derivative confirmed the calculated (large) 22 differences in barriers between electrocyclic and sigmatropic pathways.

24 Endiandric acids, phytochemicals that were first discovered by ${ }_{25}$ Gatehouse and Black, ${ }^{1,2}$ and their derivatives possess various 26 biological activities ${ }^{3}$ such as antibacterial, ${ }^{4-6}$ antitubercular, ${ }^{7}$ 27 and anticancer properties. ${ }^{6,8}$ Their biosynthesis via an intricate 28 cascade of pericyclic reactions was proposed by Black $^{2}$ and 29 verified experimentally by Nicolaou. ${ }^{9-13}$ As part of the 30 biomimetic synthesis, Nicolaou described an unexpected 31 thermal equilibrium between two bicyclo[4.2.0] octa-2,4-diene 32 intermediates, the methyl esters of the natural products 33 endiandric acid D and endiandric acid E (Scheme 1,

Scheme 1. Thermal Rearrangement of Endiandric Acids D and E: Electrocyclic Route
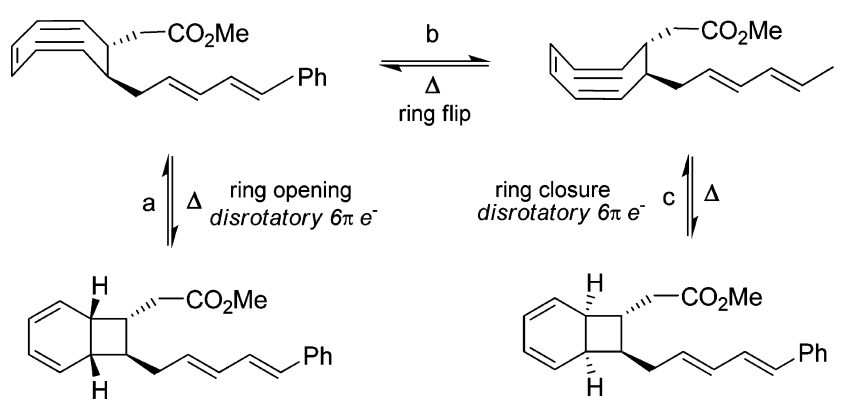

Endiandric Acid D methyl ester 1

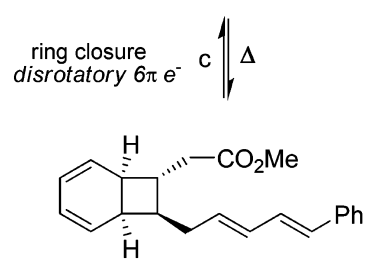

Endiandric Acid E methyl ester 2 compounds 1 and 2, respectively), and proposed a three-step 34 electrocyclic cascade for this equilibrium via (a) an electrocyclic 35 ring opening followed by (b) a ring flip of the resulting 36 cyclooctatriene (COT) and (c) a subsequent electrocyclization. 37

However, an alternative sigmatropic mechanism for this 38 thermal rearrangement via a $[1,5]$ carbon shift might be 39 possible at high temperatures (Scheme 2).

$40 \mathrm{~s} 2$

Most sigmatropic [1,5] hydrogen migrations (Scheme 3, I, R 41 s3 $=\mathrm{H})$ are pericyclic transformations, which typically possess 42 relatively high activation barriers and thus usually require high 43 reaction temperatures. ${ }^{14-16}$ Pericyclic reactions are important 44 both from a synthetic and a theoretical point of view, ${ }^{17,18}$ due 45

Scheme 2. Thermal Rearrangement of Endiandric Acids D and E via a Concerted Sigmatropic Route (Transition State on the Left) or a Stepwise Sigmatropic Route (Biradical Intermediate on the Right)

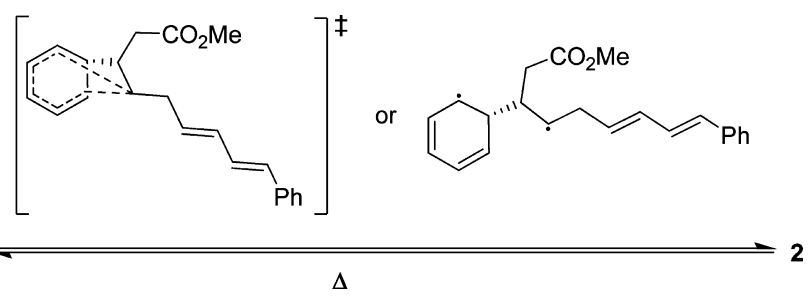

Received: December 6, 2014 
Scheme 3. [1,5] Sigmatropic Rearrangements of (I) 1,3-Dienes; (II) Bicyclo[4.1.0]hepta-2,4-dienes; and (III) Bicyclo[4.2.0] octa-2,4-dienes

(I)<smiles>[R]C/C=C\C=C/C=C\C=C/C=C\C</smiles>

(II)<smiles>C1=CC=CCC=C1</smiles><smiles>CC1C=C2CC2CC1C</smiles>

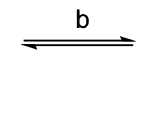

(III)
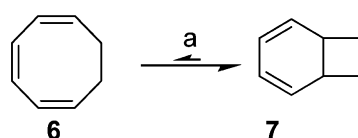

$$
\begin{gathered}
\mathrm{R}=\mathrm{H} ; \mathrm{E}_{\mathrm{a}}=30-40 \mathrm{kcal} / \mathrm{mol}^{14} \\
\mathrm{R}=\mathrm{alkyl} ; \mathrm{E}_{\mathrm{a}}=35-40 \mathrm{kcal} / \mathrm{mol}^{14}
\end{gathered}
$$

$\mathrm{E}_{\mathrm{a}}(4 \rightarrow 5)=35-45 \mathrm{kcal} / \mathrm{mol}^{38,39}$
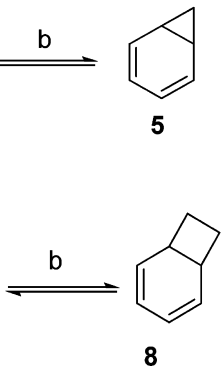

$\mathrm{E}_{\mathrm{a}}(7 \rightarrow 8)=?$
46 to their highly ordered transition states, these concerted 47 transformations usually offer a high degree of selectivity and a 48 high level of mechanistic insight. ${ }^{19,20}$ Different types of 49 observed and hypothetical pericyclic processes have been very 50 efficiently categorized depending on the nature of the 51 interacting molecular orbitals. Moreover, consideration of the 52 required symmetry of the implicated orbitals leads to a 53 straightforward prediction of a specific transformation being 54 "favored" or "disfavored". ${ }^{21-24}$ However, whether a pericyclic 55 process is a viable reaction pathway, depends on a complex 56 interplay of many factors, and therefore, it is often difficult to 57 make reliable predictions.

58 Sigmatropic $[1, n]$ carbon migrations (Scheme 3, I, R = alkyl) 59 on the other hand, do not generally involve concerted 60 transition states because the overlap of the orbitals in the 61 transition structure is usually too weak ${ }^{25}$ but are believed to 62 occur via intermediate singlet-state biradicals. ${ }^{26,27}$ Exceptions, 63 involving pericyclic transition states with good overlap, are the $64[1,5]$ sigmatropic migration in 1,3-cyclopentadienes ${ }^{28,29}$ and 65 the so-called "walk rearrangements" ${ }^{30-34}$ of bicyclo[n.1.0]66 polyenes for which the thermally allowed process should occur 67 with inversion of configuration at the migrating carbon 68 atom. ${ }^{27,35,36}$ Walk rearrangements are $[1,5]$ sigmatropic shifts 69 which involve the migration of a divalent group (O, S, NR, or $\left.{ }_{70} \mathrm{CR}_{2}\right)$ that is part of a three-membered ring in a bicyclic system 71 (Scheme 3, II, for $\mathrm{CH}_{2}$ ). These thermally induced processes 72 have been demonstrated in various bicyclo[n.1.0]polyene 73 structures.

74 Thermal rearrangements of bicyclo[4.1.0]hepta-2,4-diene 4 75 (or norcaradiene, Scheme 3, II) systems have received a lot of 76 attention in both experimental and computational stud77 ies, ${ }^{17,27,31,32,37-39}$ as they have been observed to proceed with 78 inversion at the migrating center, indicating an orbital79 symmetry forbidden rearrangement. ${ }^{27,40,41}$ However, these 80 reactions have been shown not to be concerted and thus not 81 subject to the rules of orbital symmetry conservation. ${ }^{27}$

82 The experimentally determined activation energies for 83 various substituted norcaradiene walk rearrangements do not 84 differ significantly from those of normal $[1,5]$ alkyl shifts 85 (Scheme 3, II and I with R = alkyl, respectively). ${ }^{38}$ This can be 86 rationalized by the fact that the norcaradiene system $\mathbf{4}$ is usually 87 the less populated valence tautomer in a $6 \pi$ electrocyclization 88 equilibrium with a less constrained cycloheptatriene 3 (IIa), 89 adding to the overall barrier for the carbon shift. However, a 90 different situation exists for the homologous bicyclo[4.2.0] octa- 2,4-diene (Scheme 3, III), where the electrocyclization product 91 7 is known to be favored over the contorted cyclooctatriene 692 form in most cases (IIIa). ${ }^{42}$

\section{3}

Although there is no prior literature of walk rearrangements 94 in ethylene-bridged cyclic polyene systems, in the context of the 95 well-documented similarity in the reactivity of vinyl cyclo- 96 propane and vinyl cyclobutane systems in their formal [1,3] 97 carbon shifts to a cyclopentene and a cyclohexene system, 98 respectively (Scheme 4, I and II, respectively), ${ }^{43-47}$ at high 9954

Scheme 4. [1,3] Sigmatropic Rearrangements of (I) Vinyl Cyclopropane and (II) Vinyl Cyclobutane

(I)

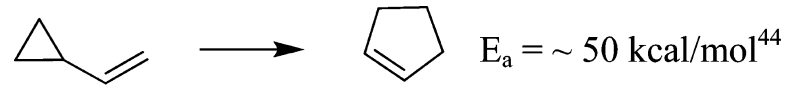

(II)

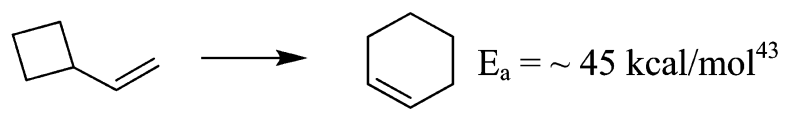

temperatures, a ring walk-type $[1,5]$ carbon shift in a 100 bicyclo[4.2.0] octa-2,4-diene (Scheme 3, IIIb) system seems to 101 be a viable reaction pathway on the basis of the norcaradiene 102 precedent.

As there is no straightforward way to distinguish 104 experimentally between these two mechanistic schemes 105 (electrocyclic versus sigmatropic) in this particular case, both 106 rearrangement pathways have been comparatively studied from 107 a theoretical point of view.

Additionally, in order to verify theoretical results, an 109 experiment using a model bicyclo[4.2.0]octa-2,4-diene system 110 9 (Scheme 5) has been devised. Due to the pseudo- $C_{2} 111$ s5 symmetry of this system, the interconverting structures (with 112 respect to their Endiandric acid D and E counterparts) are 113 identical (Scheme 5a). However, this model system is readily 114 accessible as the deuterium labeled analog $\mathbf{9}-\mathbf{d}_{\mathbf{4}}$. The thermal 115 rearrangement of diol $9-\mathrm{d}_{4}$ would only be unnoticed if it 116 proceeds exclusively via the electrocyclic route. A sigmatropic 117 pathway (or walk rearrangement) would lead to different 118 products with respect to their deuterium substitution patterns 119 (Scheme 5b).

Thus, the aim of this study is 2-fold: unraveling the 121 mechanism of thermal equilibration between endiandric acid 122 methyl esters $\mathrm{D} / \mathrm{E}$ in particular and more generally exploring 123 
Scheme 5. Thermal Rearrangements of a (a) Model Diol System 9 and its (b) Deuterium-Labeled Analog 9-d

(a)<smiles>OC[C@H]1[C@@H]2C=CC=C[C@@H]2[C@H]1CO</smiles>

9 electrocyclic<smiles>[O-]</smiles>

or

sigmatropic<smiles>OC[C@H]1[C@@H]2C=CC=C[C@H]2[C@H]1CO</smiles>

$10=9$

(b)

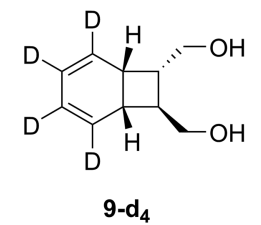

[1,5]<smiles>[2H]C1=CC2([2H])C([2H])=C([2H])C(CO)[C@@H]2[C@H](CO)[C@H]1CO</smiles>

$[1,5]$<smiles>[2H]C1=CC=CC2([2H])[C@@H](CO)[C@@H](CO)[C@]2([2H])C1[2H]</smiles>

n.s

\section{ELECTROCYCLIC PROCESS}

(a)<smiles>[R]C1[C@H]([R])[C@]2(C)C(C)=CC=C[C@@H]12</smiles>

9

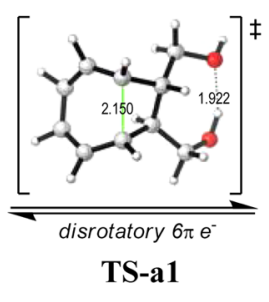

TS-a1

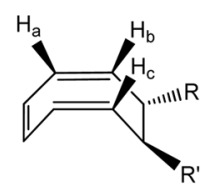

Int-a1

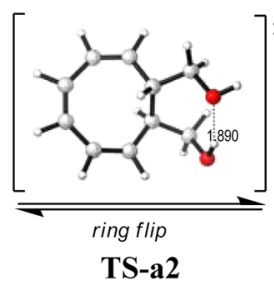

TS-a2

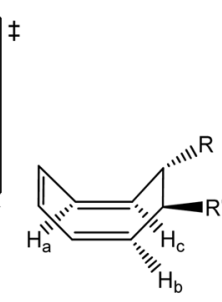

Int-a2

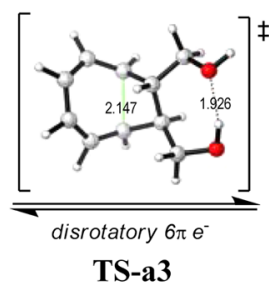

TS-a3

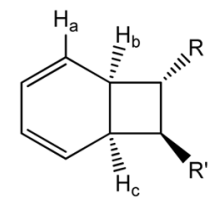

10

\section{SIGMATROPIC PROCESS}

(b)
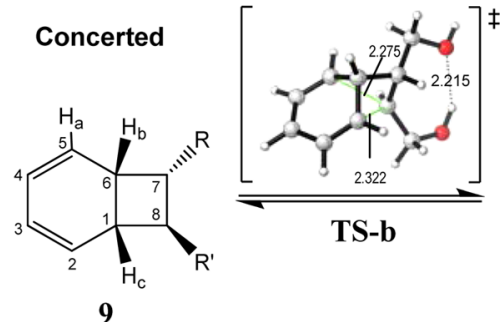<smiles>[R]C1[C@H]([2H])[C@]2(C)C=CC=C[C@]12C</smiles>

10

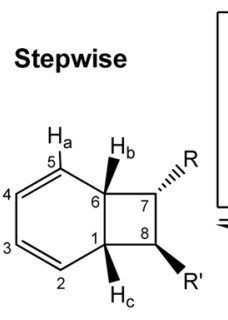

9

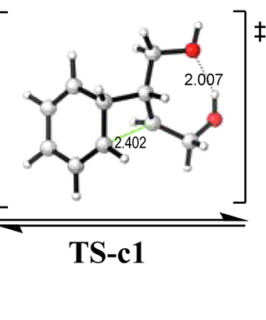<smiles>[R]CC([R])C1(C)CC=CCC1C</smiles>

Int-c

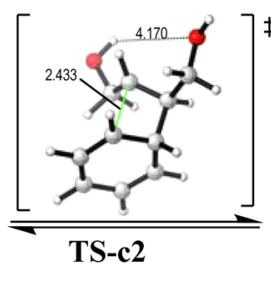

10

Figure 1. Schematic representation of the electrocyclic and sigmatropic mechanisms for the thermal equilibration of bicyclo[4.2.0] octa-2,4-diene 9. (a) $\mathrm{R}=\mathrm{R}^{\prime}=-\mathrm{CH}_{2} \mathrm{OH}$. (b) M06-2X/6-31+G(d,p) geometries for pathway a and UM06-2X/6-31+G(d,p) geometries for pathways b and c. (c) Distances in angstroms.

124 the possibility of $[1,5]$ sigmatropic alkyl shifts (walk rearrange125 ments) in bicyclo[4.2.0] octa-2,4-diene systems at high temper126 atures through a combined computational and experimental 127 study.

\section{COMPUTATIONAL METHODOLOGY}

129 All reactants, transition states, intermediates, and products were 130 optimized using three different functionals with a $6-31+G(d, p)$ basis set: ${ }^{48,49}$ the well-established hybrid functional B3LYP, ${ }^{50,51} 131$ Truhlar's meta hybrid exchange-correlation functional M06- 132 $2 \mathrm{X},{ }^{52,53}$ which accounts for dispersion, and Grimme's B3LYP- 133 D3 approach, ${ }^{54}$ which takes into account van der Waals 134 interactions by empirically adding long-range dispersive 135 corrections. ${ }^{55}$ Harmonic vibrational frequencies were computed 136 at the same levels of theory and used to provide thermal 137 corrections to the Gibbs free energies and to confirm the nature 138 of the stationary points. The intrinsic reaction coordinate 139 


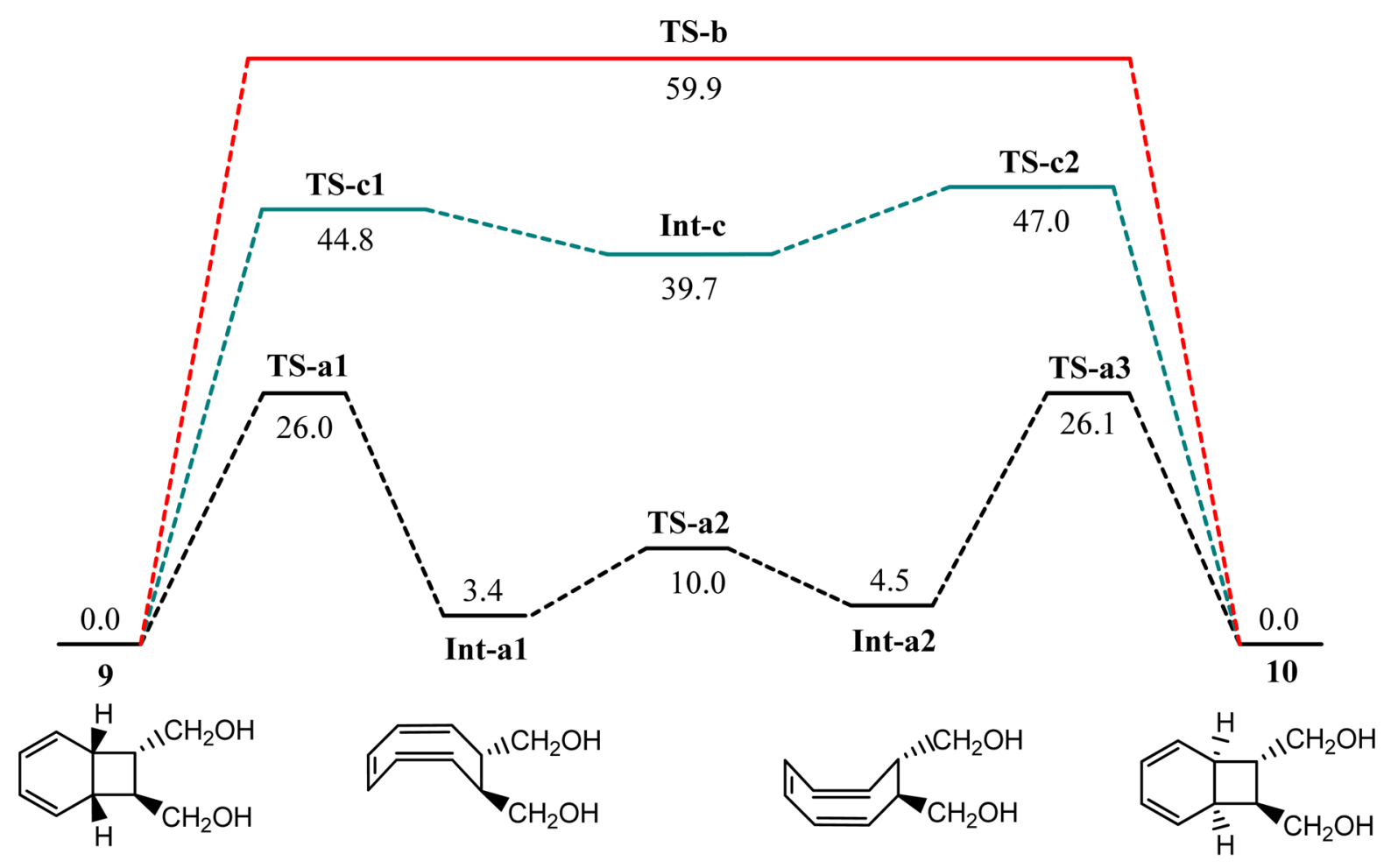

- Electrocyclic

Sigmatropic concerted

Sigmatropic stepwise

Figure 2. Free-energy profiles for the electrocyclic (M06-2X/6-31+G(d,p)) and sigmatropic pathways (UM06-2X/6-31+G(d,p)) for the thermal equilibration of bicyclo[4.2.0] octa-2,4-diene 9. Energies in kilocalories per mol.

$140(\text { IRC })^{56,57}$ paths were traced to verify the two associated 141 minima connected to each transition state on the potential 142 energy surfaces. In order to investigate the possibility of open143 shell transition states and an open-shell biradical intermediate 144 for the sigmatropic processes, HOMO and LUMO initial 145 guesses were mixed to produce unrestricted wave functions for 146 singlet states, and the stability of the wave functions was 147 checked. ${ }^{58,59}$ These calculations were carried out with Gaussian $14809 .{ }^{60}$ In order to assess the diradical character, CASSCF/6$14931+\mathrm{G}(\mathrm{d}, \mathrm{p})$ calculations were carried out on M06-2X optimized 150 structures. ${ }^{61}$ An active space of ROHF molecular orbitals with 151 all valence electrons was targeted with the density matrix 152 renormalization group (DMRG), ${ }^{62,63}$ which yielded approx153 imate natural orbitals. On the basis of the natural orbital 154 occupation numbers (NOON), the active space for the 155 subsequent CASSCF calculations was identified: natural 156 orbitals with $0.01<\mathrm{NOON}<1.99$ were regarded as essential 157 for the CASSCF calculations. We refer the reader to ref 64 for 158 an introduction to this procedure, which yields an unbiased 159 initial orbital guess. Both the DMRG and CASSCF calculations 160 were carried out with the free open-source ab initio DMRG 161 code CHEMPS2. ${ }^{65,66}$ For the initial DMRG rotation to 162 approximate natural orbitals, $D_{\mathrm{SU}(2)}=750$ reduced renormal163 ized basis states were retained. In order to obtain Gibbs free 164 CASSCF energies, thermal free energy corrections were taken 165 from the M06-2X optimizations.

\section{RESULTS AND DISCUSSION}

167 Electrocyclic and sigmatropic pathways were computationally 168 explored for the thermal equilibration of three different 169 bicyclo[4.2.0] octa-2,4-diene systems. Computational results 170 were compared with relevant literature data where applicable.
The possibility of $[1,5]$ sigmatropic alkyl shifts (walk 171 rearrangements) at high temperatures was also experimentally 172 explored.

173

1. Thermal Equilibration of Bicyclo[4.2.0]octa-2,4- 174 diene 9. The thermal equilibration of bicyclo[4.2.0] octa-2,4- 175 diene 9 via electrocyclic and sigmatropic (concerted and 176 stepwise) pathways was explored in a combined computational 177 and experimental study.

A. Theoretical Study. Initially, an electrocyclic pathway via 179 an electrocyclic ring opening followed by a ring flip and a 180 subsequent electrocyclization, which was proposed by Nicolaou 181 for endiandric acids D and $\mathrm{E}^{9-13}$ was studied computationally 182 for bicyclo[4.2.0] octa-2,4-diene 9 (Figure 1, pathway a). $183 \mathrm{fl}$

Orbital symmetry selection rules state that "allowed" 184 sigmatropic reactions occur through concerted pathways, as 185 opposed to "forbidden" processes that are known to thermally 186 occur via stepwise pathways, which go through biradical 187 intermediates. $^{55 \mathrm{c}}$ However, it has been shown that stepwise 188 routes may be favored over concerted ones for some orbital 189 symmetry allowed processes, where substituents stabilize the 190 intermediate biradical. ${ }^{19,67-69}$ For this reason, the thermal $[1,5] 191$ sigmatropic carbon shift under study has been explored through 192 both a concerted and a biradical-mediated stepwise pathway 193 (Figure 1, pathways b and c, respectively).

194

Electrocyclic Conversion of Bicyclo[4.2.0]octa-2,4-diene 9. 195 Figure 1 depicts a schematic representation along with 196 optimized transition state geometries for the electrocyclic 197 pathway of the thermal equilibration of bicyclo[4.2.0] octa-2,4- 198 diene 9 (pathway a). Furthermore, the free-energy profile is 199 shown in Figure 2. The first step in the electrocyclic process is $200 \mathrm{f} 2$ the ring opening of 9 via $\mathrm{C} 1-\mathrm{C} 6$ bond cleavage through 201 transition state TS-a1. This early transition state has a C1-C6 202 
203 bond elongation that is relatively small (2.150 $\AA$ compared to 2041.558 and $3.081 \AA$ for reactant 9 and intermediate Int-a 1, 205 respectively) and the Gibbs free activation barrier $\left(\Delta G^{\ddagger}\right)$ for 206 this step is $26.0 \mathrm{kcal} / \mathrm{mol}$ at the M06-2X/6-31+G(d,p) level of 207 theory. The ring opening leads to a contorted cyclooctatriene 208 intermediate Int-a1, which subsequently undergoes a ring flip 209 through transition state TS-a2. This second step is charac210 terized by a $\Delta G^{\ddagger}$ of only $6.6 \mathrm{kcal} / \mathrm{mol}$. Finally, electro211 cyclization through transition state TS-a3 $\left(\Delta G^{\ddagger}=26.1 \mathrm{kcal} /\right.$ $212 \mathrm{~mol}$ ) generates product 10, which is identical to the starting 213 compound 9 due to symmetry. However, retention of the 214 hydrogen bond during the reaction causes a subtle energy 215 difference between $\mathbf{9}$ and $\mathbf{1 0}$ at some levels of theory, which is 216 also the case for TS-a1 and TS-a3 and Int-a1 and Int-a3.

217 [1,5] Sigmatropic Alkyl Shift of Bicyclo[4.2.0]octa-2,4218 diene 9. The sigmatropic alkyl group shift could take place via a 219 concerted mechanism, where $\mathrm{C} 1-\mathrm{C} 8$ bond cleavage, rotation 220 of the migrating carbon around the $\mathrm{C} 6-\mathrm{C} 7$ bond and 221 formation of the new bond ( $55-\mathrm{C} 8)$ take place in a 222 synchronous concerted fashion (Figure 1, pathway $b$ ). In 223 transition state $\mathrm{TS}-\mathbf{b}$, the $\mathrm{C} 1-\mathrm{C} 8$ bond is elongated $(\mathrm{C} 1-\mathrm{C} 8$ 224 distance $2.322 \AA$ ) and a slight twist around the C6-C7 bond 225 results in an optimal position to form the new bond (C5-C8 226 distance $2.275 \AA$ ). The activation energy for this concerted 227 sigmatropic process is very high $\left(\Delta G^{\ddagger}=59.9 \mathrm{kcal} / \mathrm{mol}\right.$, UM06$2282 X / 6-31+G(d, p)$, Figure 2). Alternatively, the sigmatropic alkyl 229 group shift could take place via a biradical-mediated stepwise 230 mechanism (Figure 1, pathway c), where the first step consists 231 of homolytic $\mathrm{C} 1-\mathrm{C} 8$ bond cleavage and subsequent rotation 232 through transition state TS-c1 to the open-shell singlet 233 biradical intermediate Int-c. The transition state for this step 234 has a Gibbs free activation barrier $\Delta G^{\ddagger}$ of $44.8 \mathrm{kcal} / \mathrm{mol}$, which 235 is lower than that for the concerted sigmatropic process but still 236 quite high. The biradical intermediate Int-c (Figure 3) has a
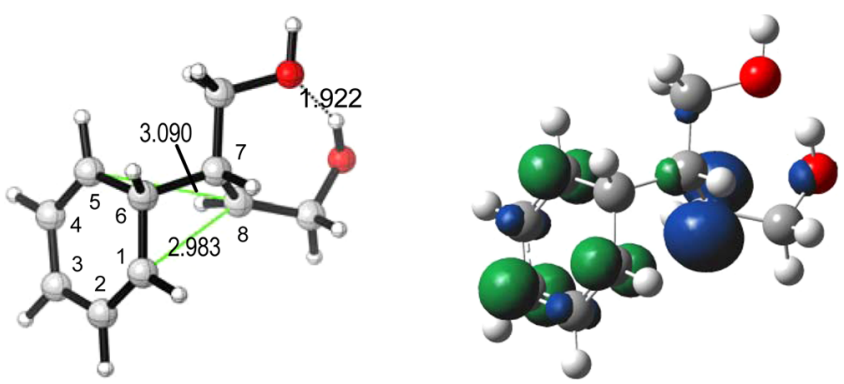

Figure 3. Open-shell singlet biradical intermediate (Int-c) in the sigmatropic stepwise process (UM06-2X/6-31+G(d,p)) for the thermal equilibration of bicyclo[4.2.0] octa-2,4-diene 9 and its isosurface (value $0.01 \mathrm{au}$ ) of spin density on the right.

237 C1-C8 distance of $2.983 \AA$ and a C5-C8 distance of $3.090 \AA$ 238 (compared to 2.322 and $2.275 \AA$ for the concerted transition 239 state TS-b). The iso-surface of the spin density for biradical 240 intermediate Int-c (Figure 3) shows that the unpaired electron 241 in the ring is delocalized not only over C1 and C5, as would be 242 expected, but also over C3, indicating the possibility of a 243 different ring closure leading to an alternative bridged product, 244 namely bicyclo[2.2.2] octa-1,5-diene, which was calculated to be $2456 \mathrm{kcal} / \mathrm{mol}$ lower in energy than product 10. This could explain 246 why complex mixtures were observed during the experiments 247 (see next section). Finally, further rotation of the exocyclic 248 radical and ring closure through transition state TS-c2 $\left(\Delta G^{\ddagger}=\right.$ $2497.3 \mathrm{kcal} / \mathrm{mol}$ ) generates product 10 .
Relative Gibbs free energies for the pathways under study, 250 calculated with three different functionals (B3LYP, M06-2X, 251 and B3LYP-D3) and a $6-31+G(d, p)$ basis set are shown in 252 Table 1. It should be noted that calculations with both the $253 \mathrm{tl}$ B3LYP and the B3LYP-D3 level of theory gave rise to an 254 internal instability of the wave function for the sigmatropic 255 concerted transition state, and analytic frequency calculations 256 are only valid if the wave function has no internal instabilities. 257 Therefore, B3LYP and B3LYP-D3 Gibbs free energies for this 258 transition state are not reported. All relative free energies 259 calculated at the M06-2X level of theory are higher than those 260 at the B3LYP level of theory. However, it can be seen that long- 261 range dispersion effects are very small in these systems, as 262 B3LYP and B3LYP-D3 values are almost equal. As expected, 263 the electrocyclic cascade is clearly preferred over the 264 sigmatropic pathways, which have much higher activation 265 barriers at all levels of theory (26.0 versus 59.9 and $44.8 \mathrm{kcal} / 266$ mol for the electrocyclic, the concerted sigmatropic and the 267 stepwise sigmatropic pathways, respectively, at the M06-2X 268 level of theory). However, the calculations predict that the 269 activation barriers for the sigmatropic process might be 270 overcome at high temperatures. Within the two sigmatropic 271 pathways, the stepwise pathway is shown to be the most 272 plausible (the activation barrier is $15.1 \mathrm{kcal} / \mathrm{mol}$ lower than for 273 the concerted pathway, M06-2X/6-31+G(d,p)).

274

Broken-symmetry unrestricted methodology was used for 275 both sigmatropic pathways, but this led to the restricted 276 solution for the concerted sigmatropic transition state TS-b, 277 suggesting a closed-shell system for this pathway, as indicated 278 by expectation values of total spin $\left\langle\mathrm{S}^{2}>\right.$ equal to zero (Table 1). 279 The stepwise sigmatropic pathway on the other hand is 280 proposed to go through open-shell transition states and a 281 corresponding open-shell singlet biradical intermediate, as 282 shown by the spin contamination $\left[\left\langle\mathrm{S}^{2}\right\rangle=0.8335,1.0372,283\right.$ and 0.7998 for TS-c1, Int-c, and TS-c2, respectively, M06-2X/ 284 $6-31+\mathrm{G}(\mathrm{d}, \mathrm{p})]$.

Since only M06-2X calculations gave rise to stable wave 286 functions for all pathways under study, further calculations were 287 done only with the M06-2X level of theory, and the CASSCF 288 calculations in the next subtopic were carried out with M06-2X 289 optimized structures.

CASSCF and DMRG Calculations. Although several 291 sigmatropic shift studies on pericyclic reactions point out that 292 inexpensive methods such as B3LYP predict activation barriers 293 and energies in excellent agreement with experimental 294 data, ${ }^{38,55 c, 70-76}$ the biradical intermediate in the stepwise 295 sigmatropic pathway implies the necessity of a multiconfigura- 296 tional self-consistent field (MCSCF) method, such as the 297 complete active space self-consistent field (CASSCF) meth- 298 $\mathrm{od}^{77}$ which was proven to be valuable for the study of organic 299 reactions. $^{78-80}$

300

With an initial approximate DMRG calculation in an active 301 space of 66 electrons in 66 ROHF molecular orbitals, which 302 contains all valence electrons, approximate natural orbitals and 303 their occupation numbers were found. Natural orbitals with 304 $0.01<\mathrm{NOON}<1.99$ were regarded as essential for the 305 CASSCF calculations, yielding a common active space of 6306 electrons in 6 orbitals.

The converged relative Gibbs free CASSCF $(6,6) / 6-31+\mathrm{G}-308$ $(\mathrm{d}, \mathrm{p})$ energies of singlet and triplet transition states and 309 intermediates for all pathways under study are shown in Table 310 1. The triplet energies are much higher than the singlet 311 energies, indicating that all pathways proceed via singlet states; 312 
Table 1. Relative Gibbs Free Energies (kcal/mol) of Reactants, Transition States, Intermediates, and Products for the Thermal Rearrangement of Bicyclo[4.2.0] octa-2,4-diene diol 9, and Expectation Values of the Total Spin $\left\langle\mathrm{S}^{2}\right\rangle$ (in Parentheses), Calculated at Different Levels of Theory (LOT) with a 6-31+G(d,p) Basis Set ${ }^{a}$

\begin{tabular}{|c|c|c|c|c|c|c|c|c|c|c|c|c|}
\hline & \multirow[b]{3}{*}{ lot } & \multirow[b]{3}{*}{9} & \multicolumn{5}{|c|}{ electrocyclic } & \multicolumn{4}{|c|}{ sigmatropic } & \multirow[b]{3}{*}{10} \\
\hline & & & \multirow[b]{2}{*}{$\begin{array}{l}\text { TS- } \\
\text { a1 }\end{array}$} & \multirow[b]{2}{*}{$\begin{array}{l}\text { Int- } \\
\text { a1 }\end{array}$} & \multirow[b]{2}{*}{$\begin{array}{l}\text { TS- } \\
\text { a2 }\end{array}$} & \multirow[b]{2}{*}{$\begin{array}{l}\text { Int- } \\
\text { a2 }\end{array}$} & \multirow[b]{2}{*}{$\begin{array}{l}\text { TS- } \\
\text { a3 }\end{array}$} & concerted & \multicolumn{3}{|c|}{ stepwise } & \\
\hline & & & & & & & & TS-b & TS-c1 & Int-c & TS-c2 & \\
\hline \multirow[t]{4}{*}{ singlet } & B3LYP & 0.0 & 22.7 & 2.4 & 6.5 & 3.2 & 22.9 & $-{ }^{b}$ & $37.7(0.7865)$ & $32.6(1.0391)$ & $38.7(0.8110)$ & 0.1 \\
\hline & B3LYP-D3 & 0.0 & 22.1 & 1.6 & 6.0 & 2.6 & 22.3 & $-{ }^{b}$ & $36.9(0.8455)$ & $33.4(1.0390)$ & $38.9(0.8227)$ & 0.1 \\
\hline & M06-2X & 0.0 & 26.0 & 3.4 & 10.0 & 4.5 & 26.1 & $59.9(0.0000)$ & $44.8(0.8335)$ & $39.7(1.0372)$ & $47.0(0.7998)$ & 0.0 \\
\hline & $\begin{array}{l}\text { CASSCF// } \\
\text { UM06-2X }\end{array}$ & 0.0 & 36.8 & 3.8 & 11.1 & 4.5 & 37.1 & 65.8 & 40.6 & 39.4 & 44.8 & 0.0 \\
\hline triplet & $\begin{array}{l}\text { CASSCF// } \\
\text { UM06- } 2 \mathrm{X}^{c, d}\end{array}$ & 54.7 & 76.0 & 49.6 & 55.0 & 50.0 & 75.9 & 146.1 & 69.7 & 38.8 & 72.2 & 56.6 \\
\hline
\end{tabular}

${ }^{a}$ Unrestricted methodology for the sigmatropic processes. ${ }^{b}$ Calculations gave rise to an internal instability of the wave function. ${ }^{c} \mathrm{CASSCF}(6,6) / 6$ $31+\mathrm{G}(\mathrm{d}, \mathrm{p}) / / \mathrm{UM} 06-2 X / 6-31+\mathrm{G}(\mathrm{d}, \mathrm{p}){ }^{d}{ }^{d}$ Energies relative to singlet reactant 9.

313 the biradical intermediate Int-c has comparable energies for its 314 singlet and triplet forms. While CASSCF and M06-2X energies 315 are in very good agreement for the sigmatropic pathways, as can 316 be seen by differences of maximum $5.9 \mathrm{kcal} / \mathrm{mol}$, differences of 317 up to $11 \mathrm{kcal} / \mathrm{mol}$ were found for the electrocyclic pathway.

318 The difference in DFT and CASSCF energetics is under319 standable, since DFT captures dynamic correlation, but not 320 static correlation, and CASSCF captures static correlation but 321 not dynamic correlation. Moreover, CASSCF indicates a closed 322 shell for the singlet and two radical electrons for the triplet in 323 the electrocyclic pathway (see Table 1 of the Supporting 324 Information), implying that single Slater determinants are able 325 to describe these structures, hence energetics from the single 326 Kohn-Sham Slater determinant in DFT calculations are 327 deemed reliable.

328 On the other hand, the converged NOON of singlet and 329 triplet transition states and intermediates (Table 1 of the 330 Supporting Information) of the sigmatropic routes, indicate 331 that all sigmatropic transition states have some diradical 332 character, and the sigmatropic stepwise intermediate is a pure 333 diradical. DFT is unable to describe these more exotic 334 electronic structures, indicating the necessity for CASPT2 335 calculations in order to get accurate energetics. ${ }^{81,82}$ However, 336 CASSCF and CASPT2 energies were shown to be comparable 337 for $[1,3]$ sigmatropic rearrangements of bicyclic and tricyclic 338 vinylcyclobutanes, ${ }^{83}$ which are described by transition states 339 highly similar in nature to the sigmatropic stepwise transition 340 states in the present study, hence the levels of theory employed 341 are considered to be sufficient. Moreover, CASSCF and M06342 X energies agree reasonably well for both sigmatropic 343 pathways.

344 DFT calculations had accurately suggested that the stepwise 345 sigmatropic pathway goes through open-shell transition states 346 and a corresponding open-shell singlet biradical intermediate; 347 however, they had incorrectly suggested the concerted 348 sigmatropic pathway to proceed through a closed-shell 349 transition state and therefore it seems to be an artifact of the 350 M06-2X calculations to give rise to stable wave functions for 351 the concerted closed-shell transition state, and therefore, no 352 further calculations will be done on this transition state.

353 As a conclusion, the electrocyclic cascade is obviously 354 preferred over the sigmatropic pathways; however, the 355 activation barriers for the stepwise sigmatropic processes 356 might be overcome at high temperatures. Moreover, DFT 357 calculations suggested that the stepwise sigmatropic pathway goes through open-shell transition states and a corresponding 358 open-shell singlet biradical intermediate and that the concerted 359 sigmatropic pathway has a closed-shell transition state. NOON 360 from CASSCF calculations on the other hand showed that all 361 sigmatropic transition states have some diradical character, and 362 the sigmatropic stepwise intermediate is a pure diradical. $\quad 363$

B. Experimental Study. In order to experimentally 364 investigate the possibility of a $[1,5]$ sigmatropic alkyl shift in 365 a bicyclo[4.2.0] octa-2,4-diene system, the diol derivative 9 was 366 synthesized in two steps from (E)-pent-2-en-4-yn-1-ol 11367 (Scheme 6). Copper-mediated oxidative Glaser coupling and $368 \mathrm{~s} 6$

Scheme 6. Synthesis of the Bicyclo[4.2.0] octa-2,4-diene diols 9 and $9-\mathrm{d}_{4}$
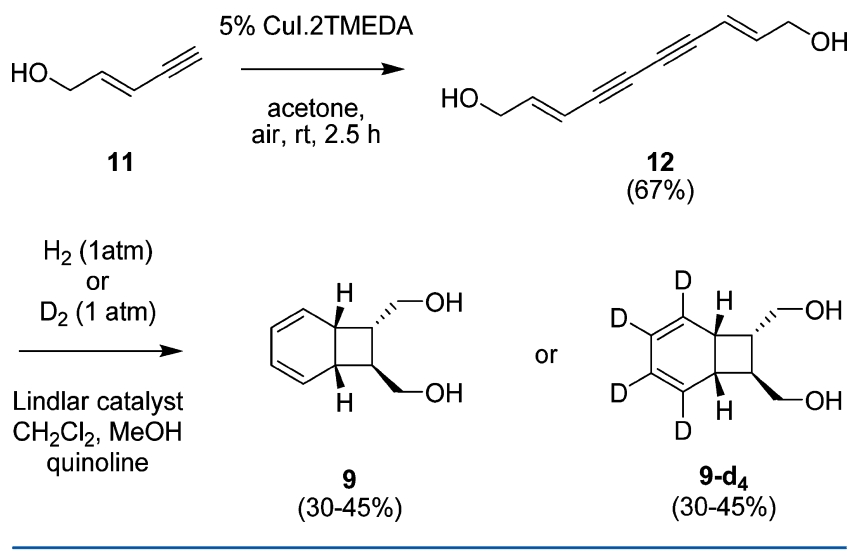

partial hydrogenation of the resulting symmetrical diyne diol 12369 was followed in situ by a cascade of an $8 \pi$ - and a $6 \pi-370$ electrocyclic ring closures as previously described, ${ }^{2,13}$ giving the 371 $4 \pi$ system 9 as the major product, in reasonable yield. When 372 the reaction was run using deuterium gas $(99.8 \%$ atom D), the 373 expected diol $\mathbf{9}-\mathbf{d}_{\mathbf{4}}$ was obtained as a single isotopomer. This 374 deuterium-labeled system was then used to study the thermal 375 rearrangements.

376

The methyl esters of endiandric acids $\mathrm{D}$ and $\mathrm{E}$ are known to 377 interconvert with a half-life of ca. $1.3 \mathrm{~h}$ at $70{ }^{\circ} \mathrm{C}$ in toluene. 378 Thus, a similar equilibrium is expected to exist in the simpler 379 diol 9, although the interconverting products are identical in 380 this case. However, for the deuterium-labeled diol $\mathbf{9}-\mathbf{d}_{\mathbf{4}}$, this 381 equilibrium would be unnoticed only if the rearrangement 382 followed exclusively the electrocyclic ring opening pathway to 383 the $6 \pi$ cyclooctatriene (COT) valence tautomer, which can 384 


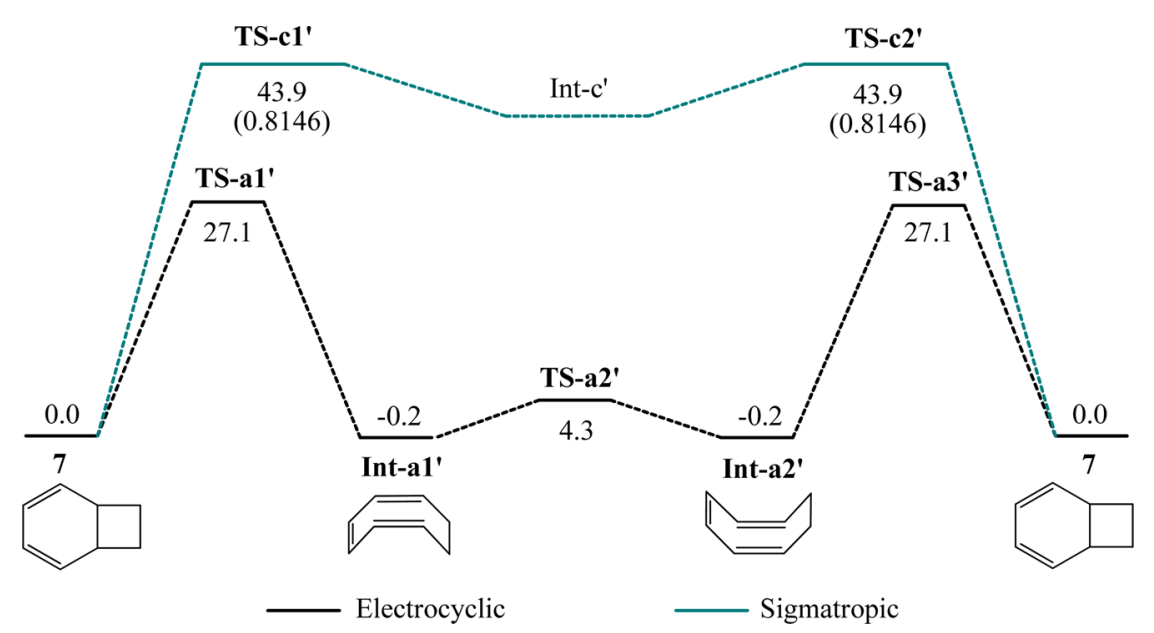

Figure 4. Free-energy profiles for the electrocyclic $(M 06-2 X / 6-31+G(d, p))$ and sigmatropic pathways (UM06-2X/6-31+G(d,p)) and expectation values of the total spin $\left\langle S^{2}\right\rangle$ (in parentheses) in the thermal equilibration of bicyclo[4.2.0] octa-2,4-diene 7. For the sigmatropic pathway, no stable intermediate could be located, and IRC paths led to an unstable intermediate that disintegrated. All energies in kilocalories per mol.

385 then ring flip and close again, whereas the alternative single step 386 pathway via a $[1,5]$ sigmatropic alkyl shift or walk rearrange387 ment would result in extensive scrambling of the deuterium 388 labels over the carbons of the six-membered ring (Scheme $5 b$ ). 389 The expected isotopomers of diol $\mathbf{9}-\mathbf{d}_{\mathbf{4}}$ should be detected 390 easily by the appearance of the diagnostic olefinic resonances in 391 the proton NMR spectrum. However, when a solution of the 392 diol $9-\mathrm{d}_{4}$ in toluene was heated at $110{ }^{\circ} \mathrm{C}$ for $1 \mathrm{~h}$, the starting 393 material was recovered unchanged by NMR analysis. 394 Consequently, at this temperature, the thermal equilibrium 395 previously described by Nicolaou and Black for the endiandric 396 acids does not constitute a walk rearrangement, as previously 397 demonstrated also by computational results.

398 When dilute solutions of $\mathbf{9}-\mathbf{d}_{\mathbf{4}}$ were heated at temperatures 399 between 170 and $195{ }^{\circ} \mathrm{C}$, olefinic resonances did appear in the 400 proton NMR spectra, which were superimposable with those 401 observed for the nonlabeled diol 9 both in $\mathrm{CDCl}_{3}$ and DMSO$402 d_{6}$. However, assignment to any of the six possible isotopomers 403 was not possible via $1 \mathrm{D}$ or $2 \mathrm{D}$ NMR experiments. Rigorous 404 chromatographic purification of the reaction mixture obtained 405 after heating for $2 \mathrm{~h}$ at $190-195{ }^{\circ} \mathrm{C}$ in acetonitrile (sealed tube) 406 gave the unchanged diol $\mathbf{9}-\mathbf{d}_{\mathbf{4}}$ as a single isotopomer in about $40730 \%$ yield. The observed olefinic resonances could therefore 408 not be explained as D-scrambled products but must arise from 409 other thermal reaction products. A similar complex mixture of 410 products was obtained when the nonlabeled diol 9 was 411 subjected to the same conditions, but none of the constituents 412 could be fully identified. Finally, heating diols 9 and $\mathbf{9}-\mathbf{d}_{\mathbf{4}}$ at 413 even higher temperatures (up to $230{ }^{\circ} \mathrm{C}$ ) in ethylene glycol 414 (sealed tube) gave a very fast $(<10 \mathrm{~min})$ and complete 415 consumption of the starting material, returning a rather 416 complex and inseparable mixture of products. In contrast to 417 most bicyclo[4.1.0] hepta-2,4-diene (norcaradiene) systems, the 418 bicyclo[4.2.0] octa-2,4-diene system appears to have limited 419 thermal stability. Furthermore, partial analysis by $2 \mathrm{D}$ NMR 420 experiments seems to implicate the completely ring-opened 421 acyclic tetraene valence tautomer as the parent structure for 422 most of the observed thermal products, a reaction pathway, 423 which is not available for the norcaradiene systems.

424 2. Thermal Equilibration of Bicyclo[4.2.0]octa-2,4425 diene 7 and Comparison with Literature. The electrocyclic 426 and sigmatropic stepwise pathways were computationally explored for the parent bicyclo[4.2.0] octa-2,4-diene compound 427 7 (Figure 4) with the M06-2X/6-31+G(d,p) level of theory, in $428 \mathrm{f} 4$ order to compare the thermal equilibration of this unsub- 429 stituted bicyclo[4.2.0] octa-2,4-diene with the norcaradiene 430 system reported in literature.

The free-energy profiles for both pathways of the thermal 432 equilibration of bicyclo[4.2.0] octa-2,4-diene 7 are shown in 433 Figure 4. These look fairly similar to the free-energy profiles for 434 bicyclo[4.2.0] octa-2,4-diene 9, again suggesting a clear 435 preference for the electrocyclic cascade. While the 436 bicyclo[4.2.0] octa-2,4-diene 9 was found to be more stable 437 than its contorted cyclooctatriene intermediate, as anticipated 438 in the introduction, this is not true for the bicyclo[4.2.0] octa- 439 2,4-diene 7, which was found to be as stable as its contorted 440 cyclooctatriene intermediate, as was also recently reported by 441 Houk. ${ }^{55 a}$ Consequently, the sigmatropic pathway is shown to 442 be less likely for bicyclo[4.2.0] octa-2,4-diene 7 when compared 443 to the bicyclo[4.2.0] octa-2,4-diene 9. Furthermore, no stable 444 singlet intermediate could be located for the unsubstituted 445 bicyclo[4.2.0] octa-2,4-diene 7, whereas an open-shell singlet 446 biradical intermediate was found for bicyclo[4.2.0] octa-2,4- 447 diene 9, which can be attributed to the difference in stability for 448 primary and secondary radicals. Nonetheless, the parent 449 bicyclo[4.2.0] octa-2,4-diene compound 7 could undergo a 450 "one step nonconcerted" sigmatropic shift, ${ }^{38,84}$ which proceeds 451 without the formation of an intermediate. 452

The Gibbs free activation barrier for the electrocyclic route 453 found here is very close to the ones reported earlier by 454 Huisgen $^{42 \mathrm{~b}}$ and recently by $\operatorname{Houk}^{55 \mathrm{a}}\left(\Delta G^{\ddagger}=27.1 \pm 0.2 \mathrm{kcal} / 455\right.$ $\mathrm{mol})$. Furthermore, although no stable singlet intermediate 456 could be located for the parent bicyclo[4.2.0] octa-2,4-diene 457 compound 7, appropriate substituents can favor the stepwise 458 sigmatropic pathway, as demonstrated in the previous section 459 for bicyclo[4.2.0] octa-2,4-diene 9. The calculated barriers for 460 the sigmatropic bond cleavage $\left(\Delta G^{\ddagger}=43.9 \mathrm{kcal} / \mathrm{mol}\right)$ are 461 within the range of experimental and predicted activation 462 barriers for [1,5] alkyl shifts in bicyclo[4.1.0] hepta-2,4-dienes, 463 which range from 35 to $45 \mathrm{kcal} / \mathrm{mol}^{38,39}$

464

As a conclusion, even though the electrocyclic cascade is 465 more plausible, comparable barriers for the [1,5] alkyl shifts of 466 bicyclo[4.2.0] octa-2,4-dienes and bicyclo[4.1.0]hepta-2,4-di- 467 enes strongly suggest that the sigmatropic stepwise pathway 468 


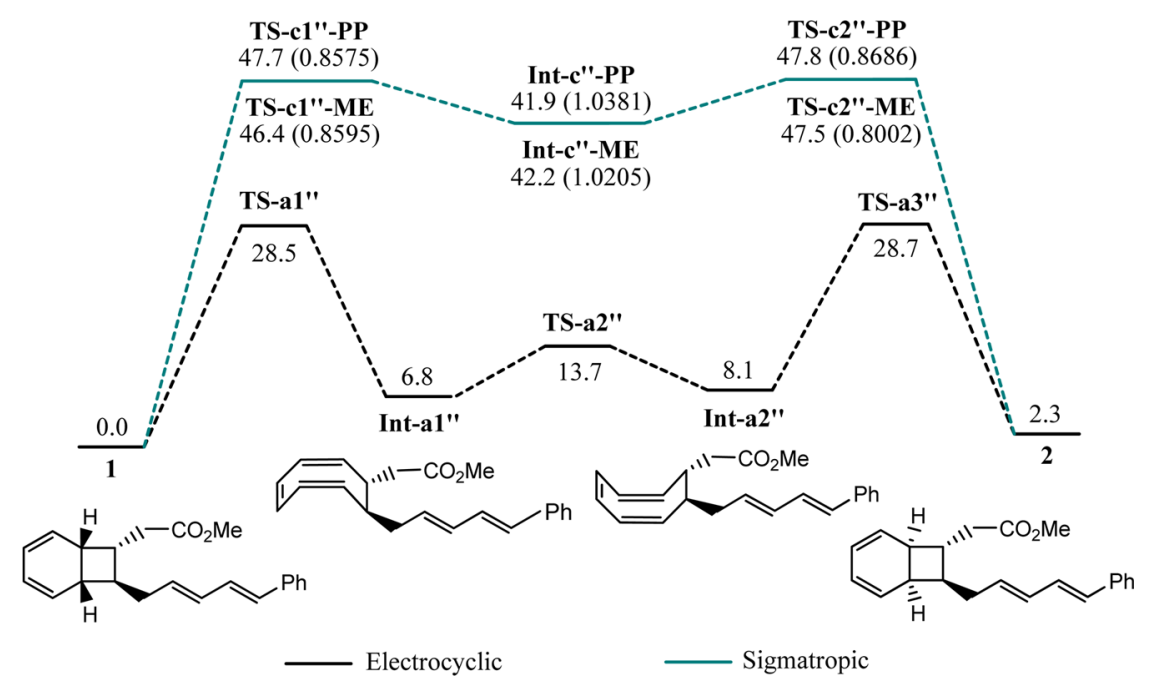

Figure 5. Free-energy profiles for the electrocyclic $(\mathrm{M} 06-2 X / 6-31+\mathrm{G}(\mathrm{d}, \mathrm{p}))$ and sigmatropic pathways (UM06-2X/6-31+G(d,p)) and expectation values of the total spin $\left\langle\mathrm{S}^{2}\right\rangle$ (in parentheses) in the thermal equilibration of endiandric acid methyl esters $\mathrm{D} / \mathrm{E}(\mathbf{1} / \mathbf{2})$. For the sigmatopic pathways, $\mathrm{PP}$ indicates the breaking of the $\mathrm{C}-\mathrm{C}$ bond close to the phenyl pentadienyl group and ME indicates the breaking of the $\mathrm{C}-\mathrm{C}$ bond close to the methyl ester group. All energies in kilocalories per mol.

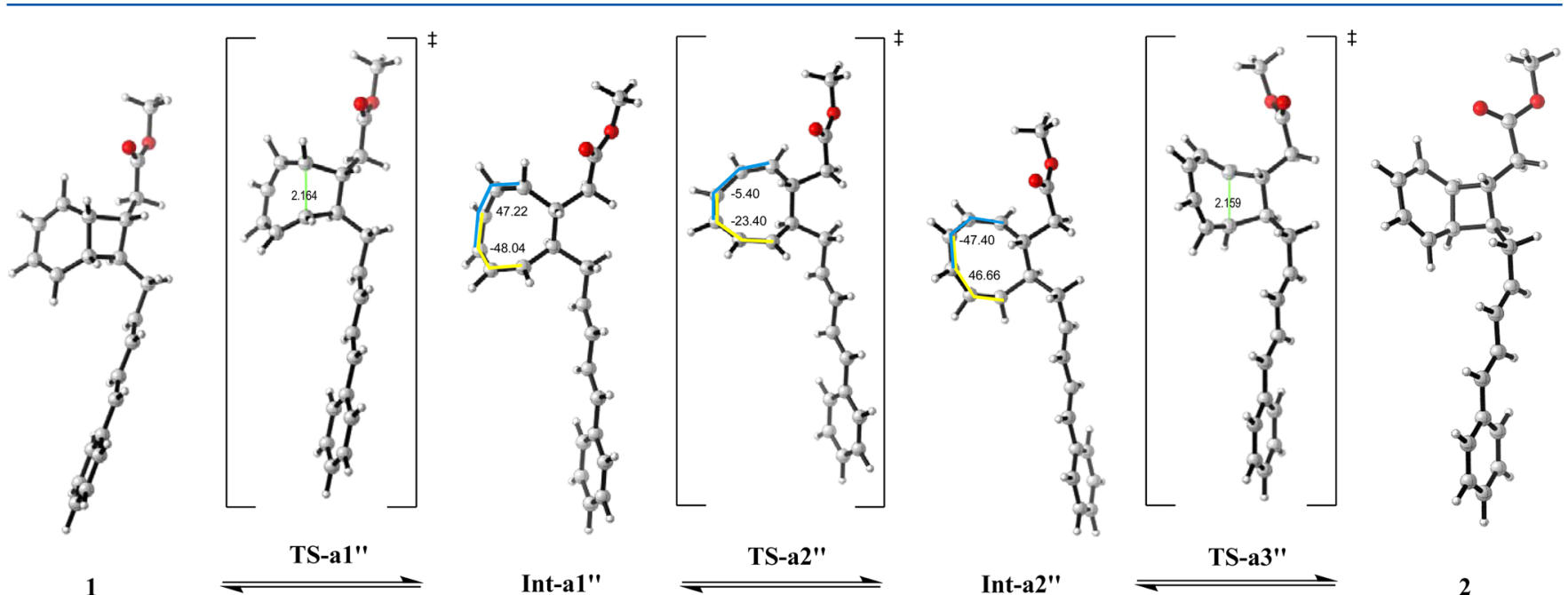

Figure 6. Electrocyclic cascade $(\mathrm{M} 06-2 X / 6-31+\mathrm{G}(\mathrm{d}, \mathrm{p}))$ for the thermal equilibration of endiandric acid methyl esters D/E (1/2). Some critical distances (green, angstroms) and dihedral angles (yellow and blue, in degrees) are shown.

469 is feasible at higher temperatures for appropriately substituted 470 compounds.

\section{3. Thermal Equilibration of Endiandric Acid Methyl}

472 Esters D/E (1/2). Finally, the electrocyclic and sigmatropic 473 stepwise pathways were computationally explored for the 474 thermal equilibration of endiandric acid methyl esters D/E (1/
475 2). The free-energy profiles shown in Figure 5 reveal relative 476 Gibbs free energies that are only slightly higher than those for 477 the thermal equilibration of the diol derivate 9 and expectation 478 values of total spin $\left\langle S^{2}>\right.$ that are comparable to those for the 479 diol derivate 9, suggesting a clear preference for the 480 electrocyclic cascade. Although the sigmatropic pathway 481 might be feasible at high temperatures. The energetically 482 favorable electrocyclic pathway along with optimized transition 483 state and intermediate geometries is shown in Figure 6. A 484 thorough conformational search was done on the phenyl 485 pentadienyl group and the methyl ester group of methyl ester $\mathbf{1}$, 486 and the most stable conformer is shown in Figure 6. All other 487 transition states and intermediates originated from this 488 conformation. Endiandric acid methyl esters $1 / 2$ were found to be more stable with respect to their contorted cyclo- 489 octatriene (COT) intermediate, compared to the diol derivate 490 9, this favors the sigmatropic pathways. Moreover, substituents 491 made it possible to locate a stable singlet intermediate for the 492 stepwise sigmatropic pathway, whereas no stable intermediate 493 could be located for the unsubstituted bicyclo[4.2.0] octa-2,4- 494 diene 7. Therefore, the stepwise sigmatropic pathway may be 495 plausible at higher temperatures for both the diol derivate $\mathbf{9}$ and 496 the endiandric acid methyl esters 1/2. Expectation values of 497 total spin $\left\langle S^{2}\right\rangle$ from DFT calculations indicate that the 498 sigmatropic pathway goes through open-shell transition states 499 and a corresponding open-shell singlet biradical intermediate 500 (Figure 5). Similarly, NOON from CASSCF calculations on the 501 diol derivate 9 had shown that the sigmatropic transition states 502 have some diradical character, and the sigmatropic intermediate 503 is a pure diradical. It was also previously shown for the 504 sigmatropic transition states of the diol derivate 9 that CASSCF 505 and M06-2X energies are in very good agreement. Since the 506 relative Gibbs free energies (M06-2X) for the thermal 507 equilibration of methyl esters $\mathbf{1} / \mathbf{2}$ are comparable to those of 508 
509 the diol derivate 9 for all pathways, CASSCF calculations were 510 not performed on the sigmatropic pathways of methyl esters 1 / 5112 , due to the large size of the system. However, this system is 512 assumed to be comparable to the diol derivate 9, which was 513 shown to proceed via singlet states for all pathways and have a 514 sigmatropic pathway with diradical character.

\section{CONCLUSION}

516 The mechanism of thermal equilibration between endiandric 517 acid methyl esters $\mathrm{D} / \mathrm{E}$ in particular and more generally the 518 possibility of $[1,5]$ sigmatropic alkyl shifts (walk rearrange519 ments) in bicyclo[4.2.0] octa-2,4-diene systems at high temper520 atures have been explored in a combined computational and 521 experimental study, pointing to the following conclusions: (a) 522 an electrocyclic cascade is clearly preferred over the sigmatropic 523 pathways; the calculated free-energy barriers for this route, 524 which was previously proposed by Nicolaou, are shown to be 525 very close to the one for bicyclo[4.2.0] octa-2,4-diene reported 526 by Huisgen. (b) The activation barriers for the sigmatropic 527 process might be overcome at high temperatures. Calculated 528 barriers for the sigmatropic stepwise pathway were shown to be 529 comparable with the reaction barriers for the bicyclo[4.1.0]530 hepta-2,4-diene (norcaradiene) walk rearrangement. Never531 theless, this stepwise pathway is only feasible for appropriately 532 substituted compounds. (c) DFT calculations suggested that 533 the stepwise sigmatropic pathway goes through open-shell 534 transition states and a corresponding open-shell singlet 535 biradical intermediate, whereas a proposed concerted sigma536 tropic pathway has a closed-shell transition state. CASSCF 537 calculations showed that all sigmatropic transition states have 538 some diradical character, and the sigmatropic stepwise 539 intermediate is a pure diradical. Therefore, the closed-shell 540 concerted sigmatropic transition state TS-b that was located 541 with DFT is not a true transition state.

542 Experimental NMR analysis on the thermal rearrangement of 543 the deuterium-labeled diol $\left(\mathbf{9}-\mathbf{d}_{\mathbf{4}}\right)$, for which the electrocyclic 544 and sigmatropic rearrangements would lead to different 545 interconverting isotopomeric products, showed that in this 546 model system, $[1,5]$ sigmatropic alkyl shifts do not occur with a 547 significant reaction rate at temperatures up to $195{ }^{\circ} \mathrm{C}$. Higher 548 temperatures could not be explored because of the limited 549 thermal stability of this bicyclic system. Our results indicate that $550[1,5]$ sigmatropic shifts should be energetically comparable 551 processes both in bicyclo[4.2.0] octa-2,4-diene and 552 bicyclo[4.1.0] hepta-2,4-diene compounds, but they have so 553 far only been observed in the latter.

\section{EXPERIMENTAL SECTION}

555 General Methods. Reactions were monitored by thin layer 556 chromatography (TLC) using UV254 precoated silicagel plates $(0.25$ $557 \mathrm{~mm}$ thickness). The TLC plates were visualized using an anisaldehyde 558 (5\% anisaldehyde in ethanol with $1 \%$ sulfuric acid) or a PMA (5\% 559 phosphomolybdic acid in ethanol) solution. Flash column chromatog560 raphy was performed using silica gel $(0.063-0.200 \mathrm{~mm}$ particle size). $561{ }^{1} \mathrm{H}$ NMR and ${ }^{13} \mathrm{C}$ NMR spectra were recorded on a $300 \mathrm{MHz}$ 562 instrument at 300 and at $75 \mathrm{MHz}$, respectively. Chemical shifts $(\delta)$ are 563 reported in units of parts per million (ppm), referenced relative to the 564 residual ${ }^{1} \mathrm{H}$ or ${ }^{13} \mathrm{C}$ peaks of the used solvent as internal standards 565 (chloroform- $d: \delta_{\mathrm{H}} 7.26$ and $\delta_{\mathrm{C}} 77.16$; dimethyl sulfoxide- $d_{6}: \delta_{\mathrm{H}} 2.49$ 566 and $\delta_{\mathrm{C}} 39.50$ ). The following abbreviations were used to explain the 567 multiplicities: s, singlet; d, doublet; $t$, triplet; $q$, quadruplet; $\mathrm{m}$, 568 multiplet; br, broadened; band, several overlapping signals; $A B, A B$ 569 system with strongly skewed signals. Where given and if appropriate, 570 assignments of resonances were confirmed by standard COSY(GPQF) and HSQC(EDETGP) 2D NMR experiments. Infrared spectra (IR) 571 were recorded on a FTIR spectrometer and reported in wave numbers 572 $\left(\mathrm{cm}^{-1}\right)$. Samples were prepared as a thin film (neat) on the $\mathrm{KBr}$ plate. 573 Mass spectra (MS) were recorded on an ESI-single quadrupole 574 detector type VL. High-Resolution Mass Spectra (HRMS) were 575 recorded on an accurate-mass quadrupole time-of-flight mass 576 spectrometer. Reported melting point ranges were determined after 577 iterative crystallization until a stable value was obtained.

Materials. All chemicals and solvents were purchased and used 579 without any further purification, except dichloromethane, which was 580 distilled from $\mathrm{CaH}_{2}$ prior to use. (E)-pent-2-en-4-yn-1-ol 11 is a 581 commercially available compound but is prohibitively expensive and 582 not readily available from standard suppliers. However, it was easily 583 prepared in two steps from ethynyl trimethylsilane.

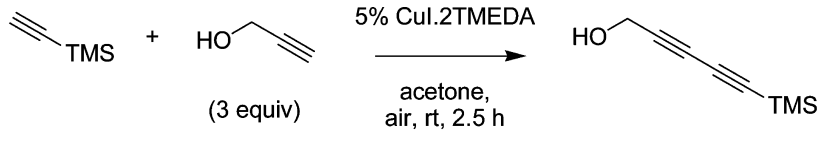

$58 \%$

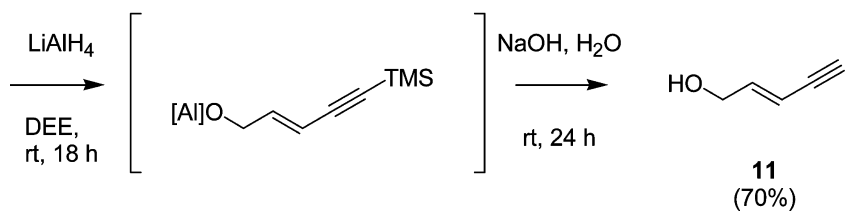

(E)-Pent-2-en-4-yn-1-ol 11. A solution of 2-propyn-1-ol (463 mg, 585 $8.3 \mathrm{mmol}$ ) and ethynyl trimethylsilane $(50 \mathrm{mg}, 0.51 \mathrm{mmol})$ in acetone 586 $(15 \mathrm{~mL})$ was added to a vigorously stirred solution of copper(I)iodide 587 $(169 \mathrm{mg}, 0.89 \mathrm{mmol})$ and tetramethylethylenediamine $(207 \mathrm{mg}, 1.78588$ $\mathrm{mmol})$ in acetone $(20 \mathrm{~mL})$, in a reaction flask that was open to air. 589 The resulting mixture was stirred open to air for $5 \mathrm{~min}$, and then a 590 solution of ethynyl trimethylsilane ( $450 \mathrm{mg}, 4.58 \mathrm{mmol}$ ) and prop-2- 591 yn-1-ol $(250 \mathrm{mg}, 4.5 \mathrm{mmol})$ in acetone $(15 \mathrm{~mL})$ was added dropwise 592 over $20 \mathrm{~min}$. The reaction was stirred for another $2 \mathrm{~h}$, the bulk of the 593 acetone was removed under reduced pressure, and the residue was 594 diluted with methyl-tert-butylether $(100 \mathrm{~mL})$. The organic layer was 595 washed with a saturated aqueous solution of ammonium chloride $(3 \times 596$ $10 \mathrm{~mL})$, water $(10 \mathrm{~mL})$, and brine $(10 \mathrm{~mL})$, dried over magnesium 597 sulfate, and concentrated in vacuo. The residue was purified by 598 chromatography on silica, eluting with 30\% methyl-tert-butylether in 599 light petroleum (bp $40-60{ }^{\circ} \mathrm{C}$ ), to give 5-trimethylsilanyl-penta-2,4- 600 diyn-1-ol (450 mg, 58\%) as a clear colorless liquid.

601

A solution of the alcohol obtained above in diethyl ether $(7.0 \mathrm{~mL}) 602$ was added dropwise over $5 \mathrm{~min}$ to a suspension of lithium 603 aluminumhydride $(333 \mathrm{mg}, 8.87 \mathrm{mmol})$ in diethyl ether $(7.0 \mathrm{~mL}) 604$ that was vigorously stirred at $0{ }^{\circ} \mathrm{C}$. The resulting mixture was warmed 605 to room temperature and stirred for $18 \mathrm{~h}$. Then, the reaction mixture 606 was cooled to $0{ }^{\circ} \mathrm{C}$, and water $(0.33 \mathrm{~mL})$ was added carefully, followed 607 by a $15 \%$ aqueous solution of sodium hydroxide $(0.33 \mathrm{~mL})$ and water 608 $(1.0 \mathrm{~mL})$. The resulting white suspension was stirred vigorously for 24609 $\mathrm{h}$, and then filtered over a pad of silica, which was thouroughly washed 610 with methyl-tert-butylether. The filtrate was concentrated to give $(E)-611$ pent-2-en-4-yn-1-ol 11 (170 mg, $70 \%)$ as a volatile, clear colorless 612 liquid which contained residual trimethyl silanol and solvent but was 613 used in the next step without further purification. The compound 614 showed proton NMR data that were consistent with data reported for 615 this compound, previously synthesized using different methods. ${ }^{85-88} 616$

(2E,8E)-Deca-2,8-diene-4,6-diyne-1,10-diol 12. The crude alcohol 617 $11(170 \mathrm{mg})$ was dissolved in acetone $(3 \mathrm{~mL})$ and then added over 5618 min to a solution of copper(I)iodide $(28 \mathrm{mg}, 0.15 \mathrm{mmol})$ and 619 tetramethylethylenediamine $(35 \mathrm{mg}, 0.30 \mathrm{mmol})$ in acetone $(3 \mathrm{~mL}) 620$ that was stirred open to air at room temperature. After stirring for 621 another $2 \mathrm{~h}$, the bulk of the acetone was removed under reduced 622 pressure, and the dark residue $(\sim 0.5 \mathrm{~mL})$ was directly subjected to 623 chromatography over silica, eluting with a 3:1 mixture of methyl-tert- 624 butylether and light petroleum (bp $40-60{ }^{\circ} \mathrm{C}$ ), to give $(2 E, 8 E)$-deca- 625 2,8-diene-4,6-diyne-1,10-diol 12 (114 mg, 67\%) as an off-white solid. 626 
$627 \mathrm{Mp} 152-153{ }^{\circ} \mathrm{C}$ (recryst. from methyl-tert-butylether and light 628 petroleum). IR $\nu_{\max }: 3284(\mathrm{~s}), 2894,2207(\mathrm{vw}) .{ }^{1} \mathrm{H} \mathrm{NMR}(300 \mathrm{MHz}$, 629 DMSO- $\left.d_{6}\right): \delta 3.18\left(4 \mathrm{H}\right.$, ddd, $J=5.4,4.3$, and $\left.1.9 \mathrm{~Hz}, 2 \times \mathrm{CH}_{2} \mathrm{OH}\right)$, $6304.17(2 \mathrm{H}, \mathrm{t}, J=5.4 \mathrm{~Hz}, 2 \times \mathrm{OH}), 4.98(2 \mathrm{H}, \mathrm{dt}, J=15.6$ and $1.9 \mathrm{~Hz}, 2$ $\left.631 \times \mathrm{CH}=\mathrm{CHCH}_{2}\right), 5.60(2 \mathrm{H}, \mathrm{dt}, J=15.6$ and $4.3 \mathrm{~Hz}, 2 \times \mathrm{CH}=$ $\left.632 \mathrm{CHCH}_{2}\right) .{ }^{13} \mathrm{C}$ NMR $\left(75 \mathrm{MHz}\right.$, DMSO- $\left.d_{6}\right): \delta 60.8\left(2 \mathrm{CH}_{2}\right), 73.5(2 \mathrm{C})$, 63380.3 (2C), $106.1(2 \mathrm{CH}), 149.1(2 \mathrm{CH}) . \mathrm{MS}(\mathrm{ESI}): m / z 145.1\left(\mathrm{MH}^{+}-\right.$ $634 \mathrm{H}_{2} \mathrm{O}$ ). HRMS (ESI) calcd. For $\mathrm{C}_{10} \mathrm{H}_{9} \mathrm{O}\left(\mathrm{m} / z \mathrm{M}+\mathrm{H}^{+}-\mathrm{H}_{2} \mathrm{O}\right)$, 635 145.0648; found, 145.0643 and calcd. For $\mathrm{C}_{10} \mathrm{H}_{11} \mathrm{O}_{2}(\mathrm{~m} / z \mathrm{M}-\mathrm{H}+$, 636 negative mode), 161.0608; found, 161.0603 .

637 (8-Hydroxymethyl-bicyclo[4.2.0]octa-2,4-dien-7-yl)-methanol 9. 638 Lindlar's catalyst (palladium, 5\% on calcium carbonate, poisoned 639 with lead (purchased from Aldrich chemical company), $102 \mathrm{mg}$ ) was 640 added to a solution of diol $12(50.0 \mathrm{mg}, 0.308 \mathrm{mmol})$ and quinoline $641(0.050 \mathrm{~mL})$ in dichloromethane $(9.0 \mathrm{~mL})$ and methanol $(1.0 \mathrm{~mL})$. 642 The resulting suspension was degassed and placed under an 643 atmosphere of hydrogen gas. The reaction progress was closely 644 monitored by thin layer chromatography. The starting material was 645 usually quickly converted into the monohydrogenated product (5-10 $646 \mathrm{~min}$ ), which was then slowly transformed into a number of products, 647 but mainly the diol $9(0.5-4 \mathrm{~h})$. The reaction mixture was degassed 648 upon consumption of the monohydrogenated intermediate (as judged 649 by TLC) and filtered over a short pad of silica, which was washed with 650 methyl-tert-butylether. The filtrate was concentrated in vacuo, and the 651 residue was purified by chromatography over silica, eluting with $2.5 \%$ 652 methanol in chloroform. The obtained product was further purified by 653 chromatography over silica, eluting with a 2:1 mixture of methyl-tert654 butylether and light petroleum (bp $40-60{ }^{\circ} \mathrm{C}$ ). This afforded the pure 655 diol $12(23.5 \mathrm{mg}, 45 \%)$ as a very viscous, clear colorless oil [in some 656 runs, lower yields were obtained (down to $30 \%)$ ). IR $\nu_{\max }: 3318(\mathrm{~s})$, $6572922(\mathrm{~s}), 1461,1376,1028 .{ }^{1} \mathrm{H}$ NMR $\left(300 \mathrm{MHz}, \mathrm{CDCl}_{3}\right): \delta 2.67-2.74$ $658(3 \mathrm{H}$, band, $3 \times$ cyclobutane $-\mathrm{CH}), 3.13-3.20(1 \mathrm{H}, \mathrm{m},=\mathrm{CH}-$ 659 CHcyclobutane), 3.45-3.51 (1H, m, CHHOH), $3.76(1 \mathrm{H}, \mathrm{dd}, J=$ 66010.2 and $3.6 \mathrm{~Hz}, \mathrm{CHHOH}), 3.79-3.85\left(2 \mathrm{H}, \mathrm{m}, \mathrm{CH}_{2} \mathrm{OH}\right), 5.53(1 \mathrm{H}$, $661 \mathrm{dd}(\mathrm{br}), J=9.7$ and $3.8 \mathrm{~Hz}, \mathrm{CH}=\mathrm{CH}-\mathrm{CH}=\mathrm{CH}), 5.60(1 \mathrm{H}, \mathrm{dd}(\mathrm{br})$, $662 J=9.5$ and $3.9 \mathrm{~Hz}, \mathrm{CH}=\mathrm{CH}-\mathrm{CH}=\mathrm{CH}), 5.72(1 \mathrm{H}, \mathrm{dd}, J=9.5$ and $6635.5 \mathrm{~Hz}, \mathrm{CH}=\mathrm{CH}-\mathrm{CH}=\mathrm{CH}), 5.87(1 \mathrm{H}, \operatorname{ddd}(\mathrm{br}), J=9.7,5.5$, and $6641.7 \mathrm{~Hz}, \mathrm{CH}=\mathrm{CH}-\mathrm{CH}=\mathrm{CH}) .{ }^{13} \mathrm{C} \mathrm{NMR}\left(75 \mathrm{MHz}, \mathrm{CDCl}_{3}\right): \delta 32.5$ $665(\mathrm{CH}), 33.0(\mathrm{CH}), 51.1(\mathrm{CH}), 52.5(\mathrm{CH}), 62.8\left(\mathrm{CH}_{2}\right), 65.5\left(\mathrm{CH}_{2}\right)$, $666122.3(\mathrm{CH}), 124.3(\mathrm{CH}), 125.5(\mathrm{CH}), 126.1(\mathrm{CH}) .{ }^{1} \mathrm{H}$ NMR $(300$ $\left.667 \mathrm{MHz}, \mathrm{DMSO}-d_{6}\right): \delta 2.25\left(1 \mathrm{H}\right.$, app. quintet, $\left.J=\sim 7.2 \mathrm{~Hz}, \mathrm{C}^{\prime}-H\right), 2.59$ $668\left(1 \mathrm{H},\left(\right.\right.$ app. q)d, $J=\sim 8.5$ and $\left.6.9 \mathrm{~Hz}, \mathrm{C} 8^{\prime}-\mathrm{H}\right), 2.66(1 \mathrm{H}, \mathrm{ddd}, J=11.1$, 6698.1 , and $\left.5.3 \mathrm{~Hz}, \mathrm{C} 6^{\prime}-H\right), 3.02\left(1 \mathrm{H}\right.$, app.t(br), $\left.J=\sim 9.5 \mathrm{~Hz}, \mathrm{Cl}^{\prime}-H\right)$, $6703.34\left(2 \mathrm{H}\right.$, app.t, $\left.\mathrm{J}=\sim 5.7 \mathrm{~Hz}, \mathrm{C} 1-\mathrm{H}_{2} \mathrm{OH}\right), 3.48(1 \mathrm{H}, \mathrm{d}(\mathrm{AB}) \mathrm{dd}, J=$ $671 \mathrm{10.4}, 6.7$, and $\left.4.5 \mathrm{~Hz}, \mathrm{C1}{ }^{\prime \prime}-\mathrm{HHOH}\right), 3.55(1 \mathrm{H}, \mathrm{d}(\mathrm{AB}) \mathrm{dd}, J=10.4,8.7$, 672 and $\left.5.3 \mathrm{~Hz}, \mathrm{Cl}^{\prime \prime}-\mathrm{HHOH}\right), 4.35\left(1 \mathrm{H}\right.$, app.t, $\left.J=\sim 4.9 \mathrm{~Hz}, \mathrm{Cl}^{\prime \prime}-\mathrm{H}_{2} \mathrm{OH}\right)$, $6734.48\left(1 \mathrm{H}\right.$, app.t, $\left.J=\sim 5.3 \mathrm{~Hz}, \mathrm{C} 1-\mathrm{H}_{2} \mathrm{OH}\right), 5.55(1 \mathrm{H}, \mathrm{d}(\mathrm{AB}) \mathrm{d}, J=9.7$ 674 and $\left.4.4 \mathrm{~Hz}, \mathrm{C5}^{\prime}-H\right)$, 5.56-5.61 $\left(1 \mathrm{H}, \mathrm{m}, \mathrm{C}^{\prime}-H\right), 5.62(1 \mathrm{H}, \mathrm{d}(\mathrm{AB}) \mathrm{d}, J$ $675=9.7$ and $\left.4.9 \mathrm{~Hz}, \mathrm{C} 4^{\prime}-H\right), 5.80(1 \mathrm{H}, \mathrm{dd}($ app.t) $) J=9.9,4.9$, and $1.5 \mathrm{~Hz}$, $\left.676 \mathrm{C}^{\prime}-\mathrm{H}\right) .{ }^{13} \mathrm{C}$ NMR $(75 \mathrm{MHz}$, DMSO-d 6 ): $\delta 32.1(\mathrm{CH}), 32.2(\mathrm{CH})$, $67748.4(\mathrm{CH}), 51.3(\mathrm{CH}), 61.2\left(\mathrm{CH}_{2}\right), 63.5\left(\mathrm{CH}_{2}\right), 121.3(\mathrm{CH}), 123.5$ $678(\mathrm{CH}), 126.1(\mathrm{CH}), 127.2(\mathrm{CH}) . \mathrm{MS}(\mathrm{ESI}): m / z 167.1\left(\mathrm{M}+\mathrm{H}^{+}, 26\right)$, $679149.1\left(\mathrm{M}+\mathrm{H}^{+}-\mathrm{H}_{2} \mathrm{O}, 100\right)$. HRMS (ESI): calcd. For $\mathrm{C}_{10} \mathrm{H}_{15} \mathrm{O}_{2}(\mathrm{~m} / z \mathrm{M}$ $\left.680+\mathrm{H}^{+}\right), 167.1067$; found, 167.1066 .

$681 \quad\left[2,3,4,5-{ }^{2} \mathrm{H}_{4}\right]$-(8-Hydroxymethyl-bicyclo[4.2.0]octa-2,4-dien-7-yl)682 methanol 9- $d_{4}$. The general procedure for the synthesis of bicyclic 683 diol 9 was followed, but deuterium gas (99.8\% atom D) was used 684 instead of hydrogen gas. Starting from $50 \mathrm{mg}$ of diol $12(0.295 \mathrm{mmol})$, 685 chromatography as described for compound 9 gave the deuterium686 labeled bicyclic diol $\mathbf{9 - \mathbf { d } _ { \mathbf { 4 } }}(18.0 \mathrm{mg}, 34 \%)$ as a clear viscous oil. IR $\nu_{\max }$ : $6873336(\mathrm{~s}), 2922(\mathrm{~s}), 1462,1376,1025 .{ }^{1} \mathrm{H}$ NMR $\left(300 \mathrm{MHz}, \mathrm{CDCl}_{3}\right): \delta$ $6882.67-2.74(3 \mathrm{H}$, band, $3 \times \mathrm{CH}), 3.14-3.19(1 \mathrm{H}, \mathrm{m},=\mathrm{CD}-\mathrm{CH})$, $6893.45-3.51(1 \mathrm{H}, \mathrm{m}, \mathrm{CHHOH}), 3.76(1 \mathrm{H}, \mathrm{dd}, J=10.2$ and $3.6 \mathrm{~Hz}$, $690 \mathrm{CHHOH}), 3.79-3.85\left(2 \mathrm{H}, \mathrm{m}, \mathrm{CH}_{2} \mathrm{OH}\right) .{ }^{13} \mathrm{C} \mathrm{NMR}(75 \mathrm{MHz}$, $\left.691 \mathrm{CDCl}_{3}\right): \delta 32.5(\mathrm{CH}), 33.0(\mathrm{CH}), 51.1(\mathrm{CH}), 52.5(\mathrm{CH}), 62.8\left(\mathrm{CH}_{2}\right)$, $69265.5\left(\mathrm{CH}_{2}\right)$. MS(ESI): $m / z 171.1\left(\mathrm{MH}^{+}, 58\right), 153.1\left(\mathrm{MH}^{+}-\mathrm{H}_{2} \mathrm{O}\right.$, 693 100). HRMS (ESI): calcd. For $\mathrm{C}_{10} \mathrm{D}_{4} \mathrm{H}_{11} \mathrm{O}_{2}\left(\mathrm{~m} / z \mathrm{M}+\mathrm{H}^{+}\right), 171.1318$, 694 found, 171.1323 .

695 Thermal Equilibration Experiments. A solution of the diol 9 or the 696 diol $9-\mathrm{d}_{4}$ (2 to $5 \mathrm{mg}$ per run) in acetonitrile $(2.0 \mathrm{~mL})$ was neutralized with $\sim 1 \mathrm{mg}$ of sodium bicarbonate and purged with argon. The 697 solution was then stirred in a closed reaction vessel under microwave 698 heating (CEM Discover). The reaction temperature and vessel 699 pressure were monitored by external surface sensors. Reactions in 700 acetonitrile were maintained at temperatures between either 170-175 701 ${ }^{\circ} \mathrm{C}$ or $190-195{ }^{\circ} \mathrm{C}$ for $1-6 \mathrm{~h}$ [which was the highest temperature that 702 could be achieved in this solvent $\left.\left(p_{\max }=17.0 \mathrm{bar}\right)\right]$. Reactions were 703 monitored by TLC and NMR, and the reaction mixtures were 704 increasingly complex with reaction temperature and time. The starting 705 materials were isolated unchanged from the reaction mixtures by 706 careful chromatography over silica, eluting with $2 \%$ methanol in 707 chloroform. The obtained products $(0.5-2.5 \mathrm{mg}, 25-50 \%)$ showed 708 ${ }^{1} \mathrm{H}$ NMR spectra which were indistinguishable from those of the 709 starting materials $\mathbf{9}$ or $\mathbf{9}-\mathbf{d}_{\mathbf{4}}$. The same experiments performed in 710 ethylene glycol, which allows reaction temperatures higher than 200711 ${ }^{\circ} \mathrm{C}$, gave similar results. However, no trace of starting material 712 remained after heating to $230{ }^{\circ} \mathrm{C}(20 \mathrm{~min})$ in these experiments, as 713 judged by TLC and NMR.

\section{ASSOCIATED CONTENT}

715

\section{S Supporting Information}

716

Cartesian coordinates and energies of M06-2X/6-31+G(d,p) 717 optimized geometries, imaginary and low frequencies of 718 transition states. Full reference 59. Natural orbital occupation 719 numbers (NOON) of transition states and intermediates for 720 the thermal rearrangement of $9(\operatorname{CASSCF}(6,6) / 6-31+G(d, p) / / 721$ UM06-2X/6-31+G(d,p)) and discussion. NMR spectra for 722 compounds $\mathbf{9}, \mathbf{9 - d _ { 4 }}, \mathbf{1 1}, \mathbf{1 2}$. This material is available free of 723 charge via the Internet at http://pubs.acs.org.

\section{AUTHOR INFORMATION}

\section{Corresponding Author}

*E-mail: saron.catak@boun.edu.tr.

Notes

The authors declare no competing financial interest.

\section{ACKNOWLEDGMENTS}

The Fund for Scientific Research Flanders (FWO) and the 731 Research Board of Ghent University are acknowledged for 732 financial support. L.H. thanks the Spanish MICIIN (MAT2011- 733 29174-C02-02) for financial support. Computational resources 734 and services used in this work were provided by Ghent 735 University.

\section{REFERENCES}

(1) Bandaranayake, W. M.; Banfield, J. E.; Black, D. S. C.; Fallon, G. 738 D.; Gatehouse, B. M. Chem. Commun. 1980, 162.

(2) Bandaranayake, W. M.; Banfield, J. E.; Black, D. S. C. Chem. 740 Commun. 1980, 902.

(3) Azmi, M. N.; Gény, C.; Leverrier, A.; Litaudon, M.; Dumontet, 742 V.; Birlirakis, N.; Guéritte, F.; Leong, K. H.; Halim, S. N. A.; 743 Mohamad, K.; Awang, K. Molecules 2014, 19, 1732.

(4) Chouna, J. R.; Nkeng-Efouet, P. A.; Lenta, B. N.; Devkota, K. P.; 745 Neumann, B.; Stammler, H.-G.; Kimbu, S. F.; Sewald, N. 746 Phytochemistry 2009, 70, 684.

(5) Chouna, J. R.; Nkeng-Efouet, P. A.; Lenta, B. N.; Wansi, J. D.; 748 Kimbu, S. F.; Sewald. Phytochem. Lett. 2010, 3, 13.

(6) Talontsi, F. M.; Lamshöft, M.; Bauer, J. O.; Razakarivony, A. A.; 750 Andriamihaja, B.; Strohmann, C.; Spiteller, M. J. Nat. Prod. 2013, 76, 751 97.

(7) Yang, P.-S.; Cheng, M.-J.; Peng, C.-F.; Chen, J.-J.; Chen, I.-S. J. 753 Nat. Prod. 2009, 72, 53.

(8) Williams, R. B.; Martin, S. M.; Hu, J.-F.; Norman, V. L.; Goering, 755 M. G.; Loss, S.; O’Neil-Johnson, M.; Eldridge, G. R.; Starks, C. M. J. 756 Nat. Prod. 2012, 75, 1319. 
758 (9) Nicolaou, K. C.; Petasis, N. A.; Zipkin, R. E.; Uenishi, J. J. Am. 759 Chem. Soc. 1982, 104, 5555.

760 (10) Nicolaou, K. C.; Petasis, N. A.; Uenishi, J.; Zipkin, R. E. J. Am. 761 Chem. Soc. 1982, 104, 5557.

762 (11) Nicolaou, K. C.; Zipkin, R. E.; Petasis, N. A. J. Am. Chem. Soc. 763 1982, 104, 5558.

764 (12) Nicolaou, K. C.; Petasis, N. A.; Zipkin, R. E. J. Am. Chem. Soc. 765 1982, 104, 5560.

766 (13) Nicolaou, K. C.; Chen, J. S. Chem. Soc. Rev. 2009, 38, 2993.

767 (14) A notable exception is the well-known [1,5] hydrogen shift of 768 cyclopentadienes, which is a fast reaction at room temperature, for 769 computational studies see: Hess, B. A.; Baldwin, J. E. J. Org. Chem. 770 2002, 67, 6025.

771 (15) Saettel, N. J.; Wiest, O. J. Org. Chem. 2000, 65, 2331.

772 (16) Alabugin, I. V.; Manoharan, M.; Breiner, B.; Lewis, F. D. J. Am. 773 Chem. Soc. 2003, 125, 9329 and references therein.

774 (17) Tantillo, D. J.; Lee, J. K. Annu. Rep. Prog. Chem., Sect. B: Org. 775 Chem. 2007, 103, 272 and refs therein.

776 (18) Beaudry, C. M.; Malerich, J. P.; Trauner, D. Chem. Rev. 2005, $777105,4757$.

778 (19) Houk, K. N.; Gonzalez, J.; Li, Y. Acc. Chem. Res. 1995, 28, 81. 779 (20) Houk, K. N.; Li, Y.; Evanseck, J. D. Angew. Chem., Int. Ed. Engl. 780 1992, 31, 682 .

781 (21) Woodward, R. B.; Hoffmann, R. J. Am. Chem. Soc. 1965, 87, 7822511 .

783 (22) Hoffmann, R.; Woodward, R. B. Acc. Chem. Res. 1968, 1, 17.

784 (23) Woodward, R. B.; Hoffmann, R. Angew. Chem., Int. Ed. Engl. $7851969,8,781$.

786 (24) Woodward, R. B.; Hoffmann, R. J. Am. Chem. Soc. 1965, 87, 395. 787 (25) Newman-Evans, R. H.; Simon, R. J.; Carpenter, B. K. J. Org. 788 Chem. 1990, 55, 695.

789 (26) Carpenter, B. K. Angew. Chem., Int. Ed. 1998, 37, 3340.

790 (27) Reyes, M. B.; Lobbovsky, E. B.; Carpenter, B. K. J. Am. Chem. 791 Soc. 2002, 124, 641 and references therein.

792 (28) Carpenter, B. K. J. Am. Chem. Soc. 1995, 117, 6336.

793 (29) Boersma, M. A. M.; De Haan, J. W.; Kloosterziel, H.; Van de 794 Ven, L. J. M. J. Chem. Soc., Chem. Commun. 1970, 1168.

795 (30) Jensen, F. J. Am. Chem. Soc. 1989, 111, 4643.

796 (31) Klärner, F. G. Top. Stereochem. 1984, 15, 1.

797 (32) Klärner, F. G. In Topics in Stereochemistry; John Wiley \& Sons, 798 Inc.: Hoboken, NJ, 2007; p 1.

799 (33) Bulo, R. E.; Jansen, H.; Ehlers, A. W.; de Kanter, F. J. J.; Schakel, 800 M.; Lutz, M.; Spek, A. L.; Lammertsma, K. Angew. Chem., Int. Ed. 801 2004, 43, 714.

802 (34) Bulo, R. E.; Allaart, F.; Ehlers, A. W.; de Kanter, F. J. J.; Schakel, 803 M.; Lutz, M.; Spek, A. L.; Lammertsma, K. J. Am. Chem. Soc. 2006, $804 \quad 128,12169$.

805 (35) Jensen, F. J. Am. Chem. Soc. 1989, 111, 4643.

806 (36) Klärner, F. G.; Wette, M. Chem. Ber. 1978, 111, 282.

807 (37) Berson, J. A.; Willcott, M. R. J. Am. Chem. Soc. 1966, 88, 2494.

808 (38) Kless, A.; Nendel, M.; Wilsey, S.; Houk, K. N. J. Am. Chem. Soc. 809 1999, 121, 4524 and refs therein.

810 (39) Jarzecki, A. A.; Gajewski, J.; Davidson, E. R. J. Am. Chem. Soc. 811 1999, 121, 6928.

812 (40) Klärner, F. G.; Brassel, B. J. Am. Chem. Soc. 1980, 102, 2469.

813 (41) Baldwin, J. E.; Broline, B. M. J. Am. Chem. Soc. 1982, 104, 2857.

814 (42) This equilibrium is mainly governed by substitution at C7 and 815 C8 positions, see: (a) Huisgen, R.; Dahmen, A.; Huber, H. J. Am. 816 Chem. Soc. 1967, 89, 7130. (b) Huisgen, R; Boche, G.; Dahmen, A.; 817 Hechtl, W. Tetrahedron Lett. 1968, 5215. (c) Fry, A. J. Tetrahedron 818 2008, 64, 2101.

819 (43) Leber, P. A.; Baldwin, J. E. Acc. Chem. Res. 2002, 35, 279.

820 (44) Baldwin, J. E. Chem. Rev. 2003, 103, 1197.

821 (45) Baldwin, J. E.; Leber, P. A. Org. Biomol. Chem. 2008, 6, 36.

822 (46) Hudlicky, T.; Reed, J. Angew. Chem., Int. Ed. 2010, 49, 4864.

823 (47) Bulo, R. E.; Ehlers, A. W.; Grimme, S.; Lammertsma, K. J. Am. 824 Chem. Soc. 2002, 124, 13903.

825 (48) Hehre, W. J.; Ditchfield, R.; Pople, J. A. J. Chem. Phys. 1972, 56, 8262257.
(49) Krishnan, R.; Binkley, J. S.; Seeger, R.; Pople, J. A. J. Chem. Phys. 827 1980, 72, 650 .

(50) Becke, A. D. J. Chem. Phys. 1993, 98, 5648.

(51) Lee, C.; Yang, W.; Parr, R. G. Phys. Rev. B 1988, 37, 785

(52) Zhao, Y.; Truhlar, D. G. Acc. Chem. Res. 2008, 41, 157.

(53) Zhao, Y.; Truhlar, D. Theor. Chem. Acc. 2008, 120, 215.

(54) Grimme, S.; Antony, J.; Ehrlich, S.; Krieg, S. J. Chem. Phys. 2010, 833 132, 154104.

(55) For literature precedents for the successful use of B3LYP and 835 M06-2X calculations on (related) electrocyclic and sigmatropic 836 reactions, we refer the reader to ref 39 and (a) Patel, A.; Houk, K. 837 N. J. Org. Chem. 2014, 79, 11370. (b) Wang, X.-N.; Krenske, E. H.; 838 Johnston, R. C.; Houk, K. N.; Hsung, R. P. J. Am. Chem. Soc. 2014, 839 136, 9802. (c) Leach, A. G.; Catak, S.; Houk, K. N. Chem.-Eur. J. 840 2002, 8, 1290.

(56) Fukui, K. Acc. Chem. Res. 1981, 14, 363.

841

(57) Hratchian, H. P.; Schlegel, H. B.; Clifford, E. D.; Frenking, G.; 843 Kwang, S. K.; Gustavo, E. S. In Theory and Applications of 844 Computational Chemistry; Elsevier: Amsterdam, 2005; p 195.

(58) Seeger, R.; Pople, J. A. J. Chem. Phys. 1977, 66, 3045.

(59) Bauernschmitt, R.; Ahlrichs, R. J. Chem. Phys. 1996, 104, 9047. 847

(60) Frisch, M. J. et al., Gaussian 09, , revision A.02; Gaussian, Inc.: 848 Wallingford, CT, 2009.

(61) For literature precedents for the successful use of CASSCF 850 calculations on related electrocyclic and sigmatropic reactions, we refer 851 the reader to ref 27, ref 38 and (a) Gutierrez, O.; Harrison, J. G.; 852 Pemberton, R. P.; Dean, J. T. Chem.-Eur. J. 2012, 18, 11029. 853 (b) Tao, H.-R.; Fang, D.-C. Theor. Chem. Acc. 2008, 121, 91.

(62) White, S. R.; Martin, R. L. J. Chem. Phys. 1999, 110, 4127.

(63) Chan, G. K.-L.; Head-Gordon, M. J. Chem. Phys. 2002, 116, 856 4462

(64) Wouters, S.; Bogaerts, T.; Van Der Voort, P.; Van Speybroeck, 858 V.; Van Neck, D. J. Chem. Phys. 2014, 140, 241103.

(65) Wouters, S.; Poelmans, W.; Ayers, P. W.; Van Neck, D. Comput. 860 Phys. Commun. 2014, 185, 1501.

(66) Wouters, S.; Van Neck, D. Eur. Phys. J. D 2014, 68, 272.

(67) Wiest, O.; Montiel, D. C.; Houk, K. N. J. Phys. Chem. A 1997, 863 $101,8378$.

(68) Hrovat, D. A.; Borden, W. T. J. Am. Chem. Soc. 2001, 123, 4069. 865

(69) Doering, W. v. E.; Ekmanis, J. L.; Belfield, K. D.; Klärner, F. G.; 866 Krawczyk, B. J. Am. Chem. Soc. 2001, 123, 5532.

(70) Guner, V.; Khuong, K. S.; Leach, A. G.; Lee, P. S.; Bartberger, 868 M. D.; Houk, K. N. J. Phys. Chem. A 2003, 107, 11445.

(71) Rodríguez-Otero, J.; Cabaleiro-Lago, E. M.; Peña-Gallego, Á. 870 Tetrahedron 2007, 63, 2191.

(72) Jursic, B. S. Comp. Theor. Chem. 1995, 358, 139.

(73) Jursic, B.; Zdravkovski, Z. J. Chem. Soc., Perkin Trans. 2 1995, 873 1223.

(74) Goldstein, E.; Beno, B.; Houk, K. N. J. Am. Chem. Soc. 1996, 875 118,6036 and references therein. 876

(75) Beno, B. R.; Wilsey, S.; Houk, K. N. J. Am. Chem. Soc. 1999, 121, 877 4816.

(76) Houk, K. N.; Beno, B. R.; Nendel, M.; Black, K.; Yoo, H. Y.; 879 Wilsey, S.; Lee, J. K. Comp. Theor. Chem. 1997, 398-399, 169.

(77) Björn, O. R. In Advances in Chemical Physics; Lawley, K. P., Ed. 881 2007; p 399.

(78) Robb, M. A.; Bernardi, F. New Theoretical Concepts for 883 Understanding Organic Reactions; Bertran, J., Czismadia, I. G., Eds. 884 Kluwer: Dordrecht, 1989.

(79) Bernardi, F.; Olivucci, M.; McDouall, J. J. W.; Robb, M. A. J. 886 Chem. Phys. 1988, 89, 6365.

(80) Bachrach, S. M. Computational Organic Chemistry; Wiley- 888 Interscience: Hoboken, NJ, 2007.

(81) Andersson, K.; Malmqvist, P. A.; Roos, B. O.; Sadlej, A. J.; 890 Wolinski, K. J. Phys. Chem. 1990, 94, 5483.

(82) Andersson, K.; Malmqvist, P.-A.; Roos, B. O. J. Chem. Phys. 892 1992, 96, 1218.

(83) Tao, H.-R.; Fang, D.-C. Theor. Chem. Acc. 2008, 121, 91.

(84) Lowe, J. P. J. Chem. Educ. 1974, 51, 785.

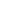
. 
896 (85) Garrais, S.; Turkington, J.; Goldring, W. P. D. Tetrahedron 2009, $89765,8418-8427$.

898 (86) Coleman, R. S.; Lu, X.; Modolo, I. J. Am. Chem. Soc. 2007, 129, 899 3826-3827.

900 (87) Yin, N.; Wang, G.; Qian, M.; Negishi, E. Angew. Chem., Int. Ed. 901 2006, 45, 2916-2920.

902 (88) Holland, D.; Stoddart, J. F. J. Chem. Soc., Perkin Trans. 1 1983, 903 1553-1571. 


\title{
Possibility of [1,5] Sigmatropic Shifts in Bicyclo[4.2.0]octa-2,4-dienes
}

\author{
Hannelore Goossens, ${ }^{\dagger}$ Johan M. Winne, $^{\ddagger}$ Sebastian Wouters, ${ }^{\dagger}$ Laura Hermosilla, ${ }^{\S}$ Pierre J. De Clercq, ${ }^{\ddagger}$ \\ Michel Waroquier, ${ }^{\dagger}$ Veronique Van Speybroeck, ${ }^{\dagger}$ and Saron Catak ${ }^{*}, \dagger, \|$
}

${ }^{\dagger}$ Center for Molecular Modeling, Ghent University, Technologiepark 903, 9052 Zwijnaarde, Belgium

${ }^{\ddagger}$ Department of Organic and Macromolecular Chemistry, Ghent University, Krijgslaan 281/S4, 9000 Ghent, Belgium

${ }^{\S}$ Departamento de Química Física Aplicada, Universidad Autónoma de Madrid, 28049 Madrid, Spain

"Department of Chemistry, Bogazici University, 34342, Bebek, Istanbul, Turkey

Supporting Information

ABSTRACT: The thermal equilibration of the methyl esters of endiandric acids $\mathrm{D}$ and $\mathrm{E}$ was subject to a computational study. An electrocyclic pathway via an electrocyclic ring opening followed by a ring flip and a subsequent electrocyclization proposed by Nicolaou [Nicolaou, K. C.; Chen, J. S. Chem. Soc. Rev. 2009, 38, 2993], was computationally explored.

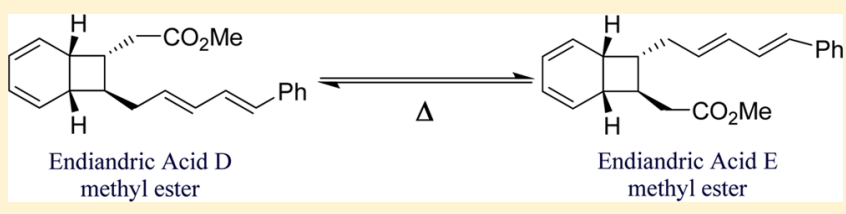

The free-energy barrier for this electrocyclic route was shown to be very close to the bicyclo[4.2.0]octa-2,4-diene reported by Huisgen [Huisgen, R.; Boche, G.; Dahmen, A.; Hechtl, W. Tetrahedron Lett. 1968, 5215]. Furthermore, the possibility of a [1,5] sigmatropic alkyl group shift of bicyclo[4.2.0] octa-2,4-diene systems at high temperatures was explored in a combined computational and experimental study. Calculated reaction barriers for an open-shell singlet biradical-mediated stepwise [1,5] sigmatropic alkyl group shift were shown to be comparable with the reaction barriers for the bicyclo[4.1.0]hepta-2,4-diene (norcaradiene) walk rearrangement. However, the stepwise sigmatropic pathway is suggested to only be feasible for appropriately substituted compounds. Experiments conducted on a deuterated analogous diol derivative confirmed the calculated (large) differences in barriers between electrocyclic and sigmatropic pathways.

\section{INTRODUCTION}

Endiandric acids, phytochemicals that were first discovered by Gatehouse and Black, ${ }^{1,2}$ and their derivatives possess various biological activities ${ }^{3}$ such as antibacterial, ${ }^{4-6}$ antitubercular, ${ }^{7}$ and anticancer properties. ${ }^{6,8}$ Their biosynthesis via an intricate cascade of pericyclic reactions was proposed by Black ${ }^{2}$ and verified experimentally by Nicolaou. ${ }^{9-13}$ As part of the biomimetic synthesis, Nicolaou described an unexpected thermal equilibrium between two bicyclo[4.2.0] octa-2,4-diene intermediates, the methyl esters of the natural products endiandric acid D and endiandric acid E (Scheme 1,

Scheme 1. Thermal Rearrangement of Endiandric Acids D and E: Electrocyclic Route

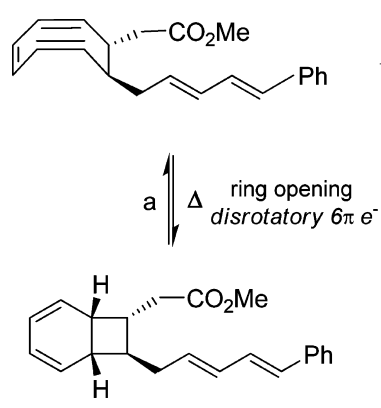

Endiandric Acid D methyl ester

1
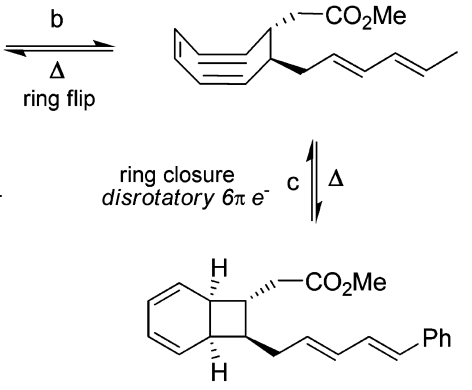

Endiandric Acid E methyl ester

2 compounds $\mathbf{1}$ and 2, respectively), and proposed a three-step electrocyclic cascade for this equilibrium via (a) an electrocyclic ring opening followed by (b) a ring flip of the resulting cyclooctatriene (COT) and (c) a subsequent electrocyclization.

However, an alternative sigmatropic mechanism for this thermal rearrangement via a $[1,5]$ carbon shift might be possible at high temperatures (Scheme 2).

Most sigmatropic $[1,5]$ hydrogen migrations (Scheme 3, I, R $=\mathrm{H})$ are pericyclic transformations, which typically possess relatively high activation barriers and thus usually require high reaction temperatures. ${ }^{14-16}$ Pericyclic reactions are important both from a synthetic and a theoretical point of view, ${ }^{17,18}$ due

Scheme 2. Thermal Rearrangement of Endiandric Acids D and E via a Concerted Sigmatropic Route (Transition State on the Left) or a Stepwise Sigmatropic Route (Biradical Intermediate on the Right)

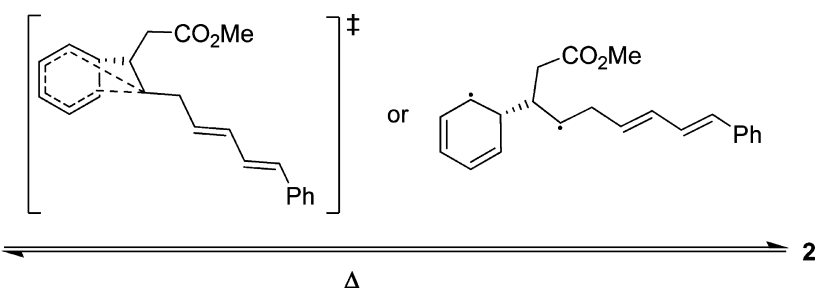

Received: December 6, 2014

Published: January 23, 2015 
Scheme 3. [1,5] Sigmatropic Rearrangements of (I) 1,3-Dienes; (II) Bicyclo[4.1.0]hepta-2,4-dienes; and (III) Bicyclo[4.2.0] octa-2,4-dienes

(I)<smiles>[R]C/C=C\C=C/C=C\C=C/C=C\C</smiles><smiles>C1=CC=CCC=C1</smiles>

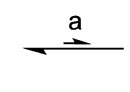<smiles>C/C=C\C=C/C</smiles>

(III)
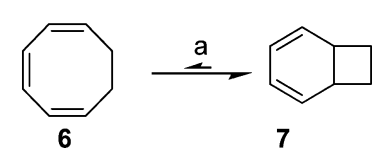

$\mathrm{R}=\mathrm{H} ; \mathrm{E}_{\mathrm{a}}=30-40 \mathrm{kcal} / \mathrm{mol}^{14}$

$\mathrm{R}=$ alkyl; $\mathrm{E}_{\mathrm{a}}=35-40 \mathrm{kcal} / \mathrm{mol}^{14}$

$\mathrm{E}_{\mathrm{a}}(4 \rightarrow 5)=35-45 \mathrm{kcal} / \mathrm{mol}^{38,39}$

$\mathrm{E}_{\mathrm{a}}(7 \rightarrow 8)=?$ to their highly ordered transition states, these concerted transformations usually offer a high degree of selectivity and a high level of mechanistic insight. ${ }^{19,20}$ Different types of observed and hypothetical pericyclic processes have been very efficiently categorized depending on the nature of the interacting molecular orbitals. Moreover, consideration of the required symmetry of the implicated orbitals leads to a straightforward prediction of a specific transformation being "favored" or "disfavored". ${ }^{21-24}$ However, whether a pericyclic process is a viable reaction pathway, depends on a complex interplay of many factors, and therefore, it is often difficult to make reliable predictions.

Sigmatropic $[1, n]$ carbon migrations (Scheme 3, I, R = alkyl) on the other hand, do not generally involve concerted transition states because the overlap of the orbitals in the transition structure is usually too weak ${ }^{25}$ but are believed to occur via intermediate singlet-state biradicals. ${ }^{26,27}$ Exceptions, involving pericyclic transition states with good overlap, are the $[1,5]$ sigmatropic migration in 1,3-cyclopentadienes ${ }^{2,29}$ and the so-called "walk rearrangements" ${ }^{30-34}$ of bicyclo[n.1.0]polyenes for which the thermally allowed process should occur with inversion of configuration at the migrating carbon atom. $^{27,35,36}$ Walk rearrangements are $[1,5]$ sigmatropic shifts which involve the migration of a divalent group $(\mathrm{O}, \mathrm{S}, \mathrm{NR}$, or $\mathrm{CR}_{2}$ ) that is part of a three-membered ring in a bicyclic system (Scheme 3, II, for $\mathrm{CH}_{2}$ ). These thermally induced processes have been demonstrated in various bicyclo[n.1.0]polyene structures.

Thermal rearrangements of bicyclo[4.1.0]hepta-2,4-diene 4 (or norcaradiene, Scheme 3, II) systems have received a lot of attention in both experimental and computational studies, ${ }^{17,27,31,32,37-39}$ as they have been observed to proceed with inversion at the migrating center, indicating an orbitalsymmetry forbidden rearrangement. ${ }^{27,40,41}$ However, these reactions have been shown not to be concerted and thus not subject to the rules of orbital symmetry conservation. ${ }^{27}$

The experimentally determined activation energies for various substituted norcaradiene walk rearrangements do not differ significantly from those of normal $[1,5]$ alkyl shifts (Scheme 3, II and I with R = alkyl, respectively). ${ }^{38}$ This can be rationalized by the fact that the norcaradiene system 4 is usually the less populated valence tautomer in a $6 \pi$ electrocyclization equilibrium with a less constrained cycloheptatriene 3 (IIa), adding to the overall barrier for the carbon shift. However, a different situation exists for the homologous bicyclo[4.2.0] octa- 2,4-diene (Scheme 3, III), where the electrocyclization product 7 is known to be favored over the contorted cyclooctatriene 6 form in most cases (IIIa). ${ }^{42}$

Although there is no prior literature of walk rearrangements in ethylene-bridged cyclic polyene systems, in the context of the well-documented similarity in the reactivity of vinyl cyclopropane and vinyl cyclobutane systems in their formal $[1,3]$ carbon shifts to a cyclopentene and a cyclohexene system, respectively (Scheme 4, I and II, respectively), ${ }^{43-47}$ at high

Scheme 4. [1,3] Sigmatropic Rearrangements of (I) Vinyl Cyclopropane and (II) Vinyl Cyclobutane

(I)

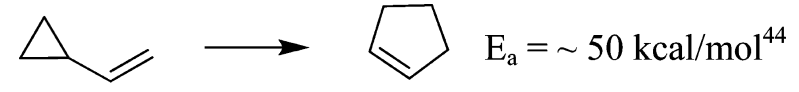

(II)

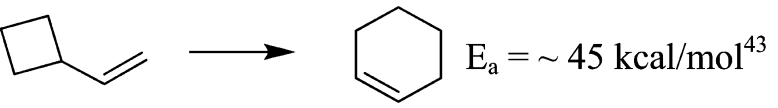

temperatures, a ring walk-type $[1,5]$ carbon shift in a bicyclo[4.2.0] octa-2,4-diene (Scheme 3, IIIb) system seems to be a viable reaction pathway on the basis of the norcaradiene precedent.

As there is no straightforward way to distinguish experimentally between these two mechanistic schemes (electrocyclic versus sigmatropic) in this particular case, both rearrangement pathways have been comparatively studied from a theoretical point of view.

Additionally, in order to verify theoretical results, an experiment using a model bicyclo[4.2.0] octa-2,4-diene system 9 (Scheme 5) has been devised. Due to the pseudo- $C_{2}$ symmetry of this system, the interconverting structures (with respect to their Endiandric acid $\mathrm{D}$ and $\mathrm{E}$ counterparts) are identical (Scheme 5a). However, this model system is readily accessible as the deuterium labeled analog $\mathbf{9}-\mathbf{d}_{\mathbf{4}}$. The thermal rearrangement of diol $\mathbf{9}-\mathrm{d}_{4}$ would only be unnoticed if it proceeds exclusively via the electrocyclic route. A sigmatropic pathway (or walk rearrangement) would lead to different products with respect to their deuterium substitution patterns (Scheme 5b).

Thus, the aim of this study is 2-fold: unraveling the mechanism of thermal equilibration between endiandric acid methyl esters $\mathrm{D} / \mathrm{E}$ in particular and more generally exploring 
Scheme 5. Thermal Rearrangements of a (a) Model Diol System 9 and its (b) Deuterium-Labeled Analog 9-d

(a)<smiles>OC[C@H]1[C@@H]2C=CC=C[C@@H]2[C@H]1CO</smiles>

9 electrocyclic<smiles>[O-]</smiles>

sigmatropic<smiles>OC[C@H]1[C@@H]2C=CC=C[C@H]2[C@H]1CO</smiles>

$10=9$

(b)<smiles>[2H]C1=C([2H])[C@@H]2[C@H](CO)[C@H](CO)[C@@H]2C([2H])=C1[2H]</smiles><smiles>[2H]C1=CC2C(CO)C(CO)C([2H])(C1[2H])C2[2H]</smiles>

[1,5]

$\rightleftharpoons$

$[1,5]$<smiles>[2H]C1=CC=CC2(CO)OC1([2H])CC(CO)C2CO</smiles>

9- $d_{4}$

\section{ELECTROCYCLIC PROCESS}

(a)<smiles>[R]C1[C@H]([R])[C@]2(C)C=CC=C[C@]12C</smiles>

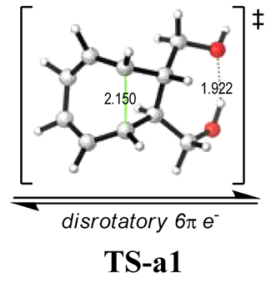

TS-a1

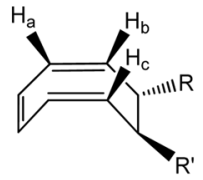

Int-a1

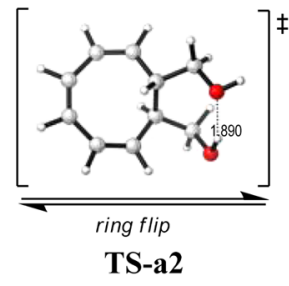

TS-a2

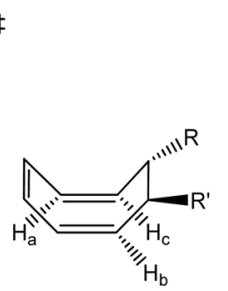

Int-a2

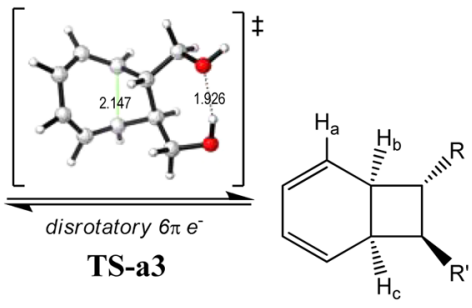

10

SIGMATROPIC PROCESS

(b)

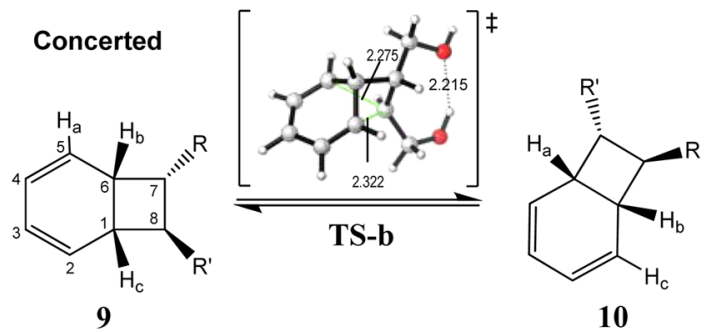

(c)<smiles>[R]C1[C@H](C)[C@@H]2C=CC=C[C@]12C</smiles>

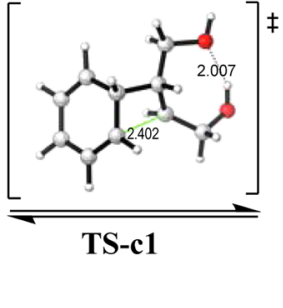

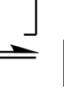$$
\text { biradical }
$$

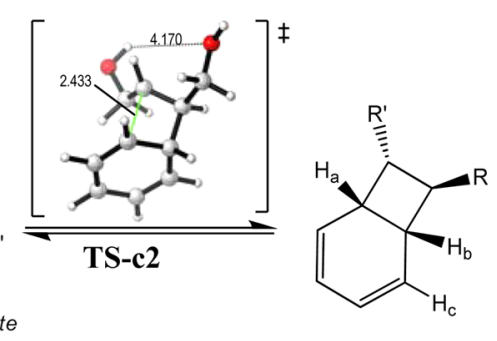

10

Figure 1. Schematic representation of the electrocyclic and sigmatropic mechanisms for the thermal equilibration of bicyclo[4.2.0] octa-2,4-diene 9. (a) $\mathrm{R}=\mathrm{R}^{\prime}=-\mathrm{CH}_{2} \mathrm{OH}$. (b) M06-2X/6-31+G(d,p) geometries for pathway a and UM06-2X/6-31+G(d,p) geometries for pathways b and c. (c) Distances in angstroms.

the possibility of $[1,5]$ sigmatropic alkyl shifts (walk rearrangements) in bicyclo[4.2.0] octa-2,4-diene systems at high temperatures through a combined computational and experimental study.

\section{COMPUTATIONAL METHODOLOGY}

All reactants, transition states, intermediates, and products were optimized using three different functionals with a $6-31+G(d, p)$ basis set: ${ }^{48,49}$ the well-established hybrid functional B3LYP, ${ }^{50,51}$ Truhlar's meta hybrid exchange-correlation functional M06$2 X, 52,53$ which accounts for dispersion, and Grimme's B3LYPD3 approach, ${ }^{54}$ which takes into account van der Waals interactions by empirically adding long-range dispersive corrections. ${ }^{55}$ Harmonic vibrational frequencies were computed at the same levels of theory and used to provide thermal corrections to the Gibbs free energies and to confirm the nature of the stationary points. The intrinsic reaction coordinate 


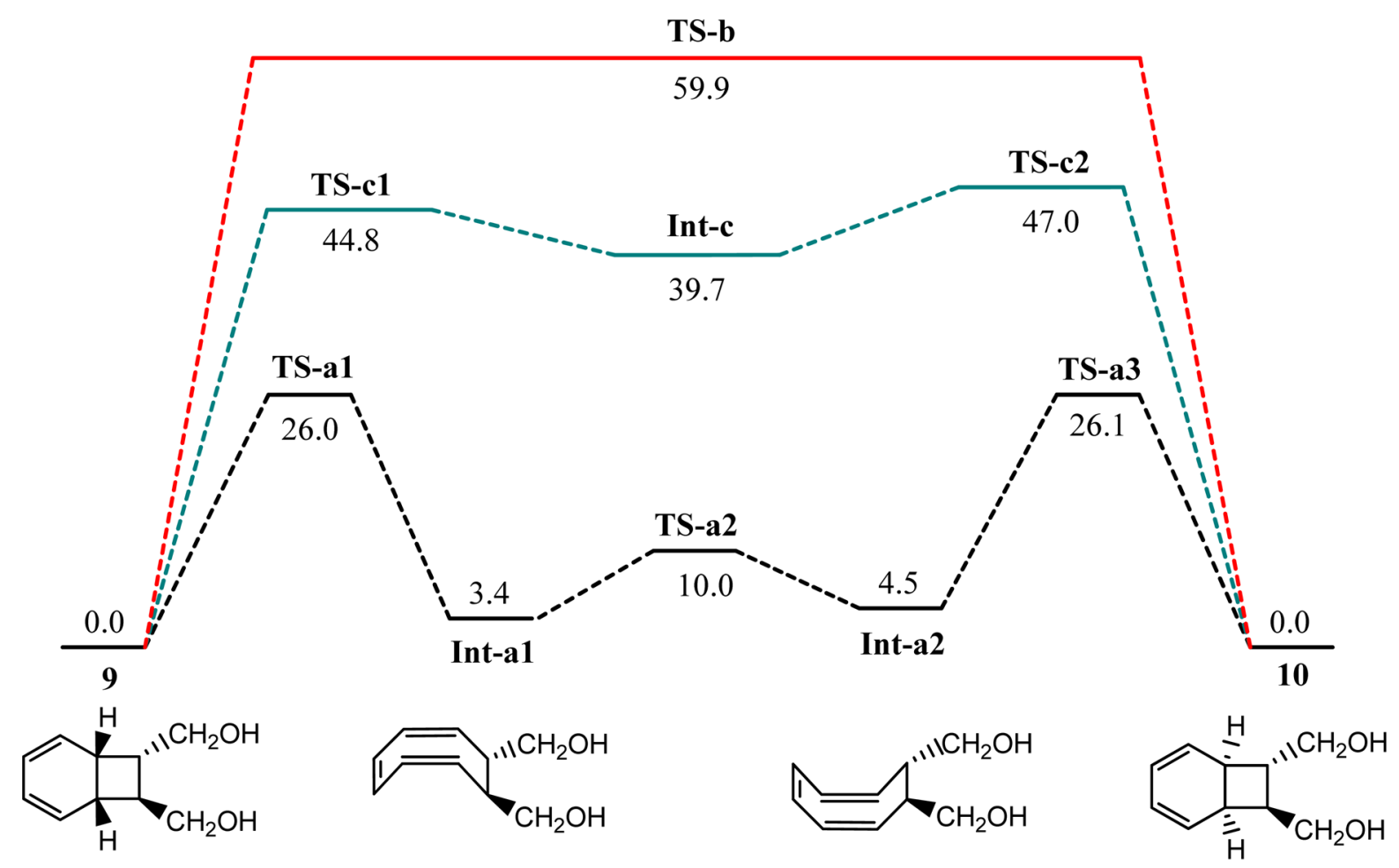

Electrocyclic - Sigmatropic concerted $\quad$ Sigmatropic stepwise

Figure 2. Free-energy profiles for the electrocyclic (M06-2X/6-31+G(d,p)) and sigmatropic pathways (UM06-2X/6-31+G(d,p)) for the thermal equilibration of bicyclo[4.2.0] octa-2,4-diene 9. Energies in kilocalories per mol.

$(\text { IRC })^{56,57}$ paths were traced to verify the two associated minima connected to each transition state on the potential energy surfaces. In order to investigate the possibility of openshell transition states and an open-shell biradical intermediate for the sigmatropic processes, HOMO and LUMO initial guesses were mixed to produce unrestricted wave functions for singlet states, and the stability of the wave functions was checked. ${ }^{58,59}$ These calculations were carried out with Gaussian $09 .^{60}$ In order to assess the diradical character, CASSCF /6$31+\mathrm{G}(\mathrm{d}, \mathrm{p})$ calculations were carried out on M06-2X optimized structures. ${ }^{61}$ An active space of ROHF molecular orbitals with all valence electrons was targeted with the density matrix renormalization group (DMRG), ${ }^{62,63}$ which yielded approximate natural orbitals. On the basis of the natural orbital occupation numbers (NOON), the active space for the subsequent CASSCF calculations was identified: natural orbitals with $0.01<\mathrm{NOON}<1.99$ were regarded as essential for the CASSCF calculations. We refer the reader to ref 64 for an introduction to this procedure, which yields an unbiased initial orbital guess. Both the DMRG and CASSCF calculations were carried out with the free open-source ab initio DMRG code CHEMPS2. ${ }^{65,66}$ For the initial DMRG rotation to approximate natural orbitals, $D_{\mathrm{SU}(2)}=750$ reduced renormalized basis states were retained. In order to obtain Gibbs free CASSCF energies, thermal free energy corrections were taken from the M06-2X optimizations.

\section{RESULTS AND DISCUSSION}

Electrocyclic and sigmatropic pathways were computationally explored for the thermal equilibration of three different bicyclo[4.2.0] octa-2,4-diene systems. Computational results were compared with relevant literature data where applicable.
The possibility of $[1,5]$ sigmatropic alkyl shifts (walk rearrangements) at high temperatures was also experimentally explored.

1. Thermal Equilibration of Bicyclo[4.2.0]octa-2,4diene 9. The thermal equilibration of bicyclo[4.2.0] octa-2,4diene 9 via electrocyclic and sigmatropic (concerted and stepwise) pathways was explored in a combined computational and experimental study.

A. Theoretical Study. Initially, an electrocyclic pathway via an electrocyclic ring opening followed by a ring flip and a subsequent electrocyclization, which was proposed by Nicolaou for endiandric acids D and $\mathrm{E},{ }^{9-13}$ was studied computationally for bicyclo[4.2.0] octa-2,4-diene 9 (Figure 1, pathway a).

Orbital symmetry selection rules state that "allowed" sigmatropic reactions occur through concerted pathways, as opposed to "forbidden" processes that are known to thermally occur via stepwise pathways, which go through biradical intermediates. $^{55 \mathrm{c}}$ However, it has been shown that stepwise routes may be favored over concerted ones for some orbital symmetry allowed processes, where substituents stabilize the intermediate biradical. ${ }^{19,67-69}$ For this reason, the thermal $[1,5]$ sigmatropic carbon shift under study has been explored through both a concerted and a biradical-mediated stepwise pathway (Figure 1, pathways b and c, respectively).

Electrocyclic Conversion of Bicyclo[4.2.0]octa-2,4-diene 9. Figure 1 depicts a schematic representation along with optimized transition state geometries for the electrocyclic pathway of the thermal equilibration of bicyclo[4.2.0] octa-2,4diene 9 (pathway a). Furthermore, the free-energy profile is shown in Figure 2. The first step in the electrocyclic process is the ring opening of 9 via $\mathrm{C} 1-\mathrm{C} 6$ bond cleavage through transition state TS-a1. This early transition state has a C1-C6 
bond elongation that is relatively small (2.150 $\AA$ compared to 1.558 and $3.081 \AA$ for reactant 9 and intermediate Int-a1, respectively) and the Gibbs free activation barrier $\left(\Delta G^{\ddagger}\right)$ for this step is $26.0 \mathrm{kcal} / \mathrm{mol}$ at the M06-2X/6-31+G(d,p) level of theory. The ring opening leads to a contorted cyclooctatriene intermediate Int-a1, which subsequently undergoes a ring flip through transition state TS-a2. This second step is characterized by a $\Delta G^{\ddagger}$ of only $6.6 \mathrm{kcal} / \mathrm{mol}$. Finally, electrocyclization through transition state TS-a3 $\left(\Delta G^{\ddagger}=26.1 \mathrm{kcal} /\right.$ mol) generates product 10, which is identical to the starting compound 9 due to symmetry. However, retention of the hydrogen bond during the reaction causes a subtle energy difference between $\mathbf{9}$ and $\mathbf{1 0}$ at some levels of theory, which is also the case for TS-a1 and TS-a3 and Int-a1 and Int-a3.

[1,5] Sigmatropic Alkyl Shift of Bicyclo[4.2.0]octa-2,4diene 9. The sigmatropic alkyl group shift could take place via a concerted mechanism, where $\mathrm{C} 1-\mathrm{C} 8$ bond cleavage, rotation of the migrating carbon around the $\mathrm{C} 6-\mathrm{C} 7$ bond and formation of the new bond (C5-C8) take place in a synchronous concerted fashion (Figure 1, pathway b). In transition state $\mathbf{T S}-\mathbf{b}$, the $\mathrm{C} 1-\mathrm{C} 8$ bond is elongated ( $\mathrm{C} 1-\mathrm{C} 8$ distance $2.322 \AA$ ) and a slight twist around the C6-C7 bond results in an optimal position to form the new bond (C5-C8 distance $2.275 \AA$ ). The activation energy for this concerted sigmatropic process is very high $\left(\Delta G^{\ddagger}=59.9 \mathrm{kcal} / \mathrm{mol}\right.$, UM06$2 X / 6-31+G(d, p)$, Figure 2). Alternatively, the sigmatropic alkyl group shift could take place via a biradical-mediated stepwise mechanism (Figure 1, pathway c), where the first step consists of homolytic $\mathrm{C} 1-\mathrm{C} 8$ bond cleavage and subsequent rotation through transition state TS-c1 to the open-shell singlet biradical intermediate Int-c. The transition state for this step has a Gibbs free activation barrier $\Delta G^{\ddagger}$ of $44.8 \mathrm{kcal} / \mathrm{mol}$, which is lower than that for the concerted sigmatropic process but still quite high. The biradical intermediate Int-c (Figure 3) has a
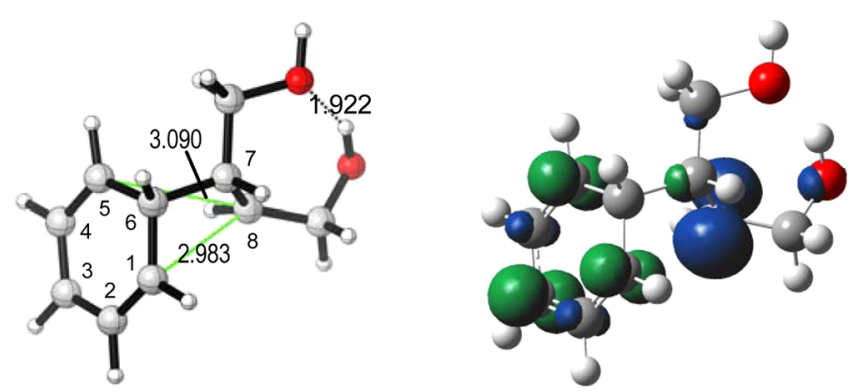

Figure 3. Open-shell singlet biradical intermediate (Int-c) in the sigmatropic stepwise process (UM06-2X/6-31+G(d,p)) for the thermal equilibration of bicyclo[4.2.0] octa-2,4-diene 9 and its isosurface (value $0.01 \mathrm{au}$ ) of spin density on the right.

C1-C8 distance of $2.983 \AA$ and a C5-C8 distance of $3.090 \AA$ (compared to 2.322 and $2.275 \AA$ for the concerted transition state TS-b). The iso-surface of the spin density for biradical intermediate Int-c (Figure 3) shows that the unpaired electron in the ring is delocalized not only over $\mathrm{C} 1$ and $\mathrm{C} 5$, as would be expected, but also over $\mathrm{C} 3$, indicating the possibility of a different ring closure leading to an alternative bridged product, namely bicyclo[2.2.2] octa-1,5-diene, which was calculated to be $6 \mathrm{kcal} / \mathrm{mol}$ lower in energy than product 10. This could explain why complex mixtures were observed during the experiments (see next section). Finally, further rotation of the exocyclic radical and ring closure through transition state TS-c2 $\left(\Delta G^{\ddagger}=\right.$ $7.3 \mathrm{kcal} / \mathrm{mol}$ ) generates product 10 .
Relative Gibbs free energies for the pathways under study, calculated with three different functionals (B3LYP, M06-2X, and B3LYP-D3) and a 6-31+G(d,p) basis set are shown in Table 1. It should be noted that calculations with both the B3LYP and the B3LYP-D3 level of theory gave rise to an internal instability of the wave function for the sigmatropic concerted transition state, and analytic frequency calculations are only valid if the wave function has no internal instabilities. Therefore, B3LYP and B3LYP-D3 Gibbs free energies for this transition state are not reported. All relative free energies calculated at the M06-2X level of theory are higher than those at the B3LYP level of theory. However, it can be seen that longrange dispersion effects are very small in these systems, as B3LYP and B3LYP-D3 values are almost equal. As expected, the electrocyclic cascade is clearly preferred over the sigmatropic pathways, which have much higher activation barriers at all levels of theory (26.0 versus 59.9 and $44.8 \mathrm{kcal} /$ mol for the electrocyclic, the concerted sigmatropic and the stepwise sigmatropic pathways, respectively, at the M06-2X level of theory). However, the calculations predict that the activation barriers for the sigmatropic process might be overcome at high temperatures. Within the two sigmatropic pathways, the stepwise pathway is shown to be the most plausible (the activation barrier is $15.1 \mathrm{kcal} / \mathrm{mol}$ lower than for the concerted pathway, M06-2X/6-31+G(d,p)).

Broken-symmetry unrestricted methodology was used for both sigmatropic pathways, but this led to the restricted solution for the concerted sigmatropic transition state TS-b, suggesting a closed-shell system for this pathway, as indicated by expectation values of total spin $<S^{2}>$ equal to zero (Table 1 ). The stepwise sigmatropic pathway on the other hand is proposed to go through open-shell transition states and a corresponding open-shell singlet biradical intermediate, as shown by the spin contamination $\left[\left\langle S^{2}\right\rangle=0.8335,1.0372\right.$, and 0.7998 for TS-c1, Int-c, and TS-c2, respectively, M06-2X/ $6-31+G(d, p)]$.

Since only M06-2X calculations gave rise to stable wave functions for all pathways under study, further calculations were done only with the M06-2X level of theory, and the CASSCF calculations in the next subtopic were carried out with M06-2X optimized structures.

CASSCF and DMRG Calculations. Although several sigmatropic shift studies on pericyclic reactions point out that inexpensive methods such as B3LYP predict activation barriers and energies in excellent agreement with experimental data, ${ }^{38,55 c, 70-76}$ the biradical intermediate in the stepwise sigmatropic pathway implies the necessity of a multiconfigurational self-consistent field (MCSCF) method, such as the complete active space self-consistent field (CASSCF) method, ${ }^{77}$ which was proven to be valuable for the study of organic reactions. ${ }^{78-80}$

With an initial approximate DMRG calculation in an active space of 66 electrons in 66 ROHF molecular orbitals, which contains all valence electrons, approximate natural orbitals and their occupation numbers were found. Natural orbitals with $0.01<\mathrm{NOON}<1.99$ were regarded as essential for the CASSCF calculations, yielding a common active space of 6 electrons in 6 orbitals.

The converged relative Gibbs free $\operatorname{CASSCF}(6,6) / 6-31+\mathrm{G}-$ $(\mathrm{d}, \mathrm{p})$ energies of singlet and triplet transition states and intermediates for all pathways under study are shown in Table 1. The triplet energies are much higher than the singlet energies, indicating that all pathways proceed via singlet states; 
Table 1. Relative Gibbs Free Energies (kcal/mol) of Reactants, Transition States, Intermediates, and Products for the Thermal Rearrangement of Bicyclo[4.2.0] octa-2,4-diene diol 9, and Expectation Values of the Total Spin $\left\langle S^{2}\right\rangle$ (in Parentheses), Calculated at Different Levels of Theory (LOT) with a 6-31+G(d,p) Basis Set ${ }^{a}$

\begin{tabular}{|c|c|c|c|c|c|c|c|c|c|c|c|c|}
\hline & \multirow[b]{3}{*}{ lot } & \multirow[b]{3}{*}{9} & \multicolumn{5}{|c|}{ electrocyclic } & \multicolumn{4}{|c|}{ sigmatropic } & \multirow[b]{3}{*}{10} \\
\hline & & & \multirow[b]{2}{*}{$\begin{array}{c}\text { TS- } \\
\text { a1 }\end{array}$} & \multirow[b]{2}{*}{$\begin{array}{l}\text { Int- } \\
\text { a } 1\end{array}$} & \multirow[b]{2}{*}{$\begin{array}{c}\text { TS- } \\
\text { a2 }\end{array}$} & \multirow[b]{2}{*}{$\begin{array}{l}\text { Int- } \\
\text { a2 }\end{array}$} & \multirow[b]{2}{*}{$\begin{array}{c}\text { TS- } \\
\text { a3 }\end{array}$} & \multirow{2}{*}{$\begin{array}{c}\text { concerted } \\
\text { TS-b }\end{array}$} & \multicolumn{3}{|c|}{ stepwise } & \\
\hline & & & & & & & & & TS-c1 & Int-c & TS-c2 & \\
\hline \multirow[t]{4}{*}{ singlet } & B3LYP & 0.0 & 22.7 & 2.4 & 6.5 & 3.2 & 22.9 & $-{ }^{b}$ & $37.7(0.7865)$ & $32.6(1.0391)$ & $38.7(0.8110)$ & 0.1 \\
\hline & B3LYP-D3 & 0.0 & 22.1 & 1.6 & 6.0 & 2.6 & 22.3 & $-{ }^{b}$ & $36.9(0.8455)$ & $33.4(1.0390)$ & $38.9(0.8227)$ & 0.1 \\
\hline & M06-2X & 0.0 & 26.0 & 3.4 & 10.0 & 4.5 & 26.1 & $59.9(0.0000)$ & $44.8(0.8335)$ & $39.7(1.0372)$ & $47.0(0.7998)$ & 0.0 \\
\hline & $\begin{array}{l}\text { CASSCF// } \\
\text { UM06-2X }\end{array}$ & 0.0 & 36.8 & 3.8 & 11.1 & 4.5 & 37.1 & 65.8 & 40.6 & 39.4 & 44.8 & 0.0 \\
\hline triplet & $\begin{array}{l}\text { CASSCF// } \\
\text { UM06- } 2 X^{c, d}\end{array}$ & 54.7 & 76.0 & 49.6 & 55.0 & 50.0 & 75.9 & 146.1 & 69.7 & 38.8 & 72.2 & 56.6 \\
\hline
\end{tabular}

${ }^{a}$ Unrestricted methodology for the sigmatropic processes. ${ }^{b}$ Calculations gave rise to an internal instability of the wave function. ${ }^{c} \mathrm{CASSCF}(6,6) / 6$ $31+\mathrm{G}(\mathrm{d}, \mathrm{p}) / / \mathrm{UM} 06-2 X / 6-31+\mathrm{G}(\mathrm{d}, \mathrm{p}) .{ }^{d}$ Energies relative to singlet reactant 9.

the biradical intermediate Int-c has comparable energies for its singlet and triplet forms. While CASSCF and M06-2X energies are in very good agreement for the sigmatropic pathways, as can be seen by differences of maximum $5.9 \mathrm{kcal} / \mathrm{mol}$, differences of up to $11 \mathrm{kcal} / \mathrm{mol}$ were found for the electrocyclic pathway.

The difference in DFT and CASSCF energetics is understandable, since DFT captures dynamic correlation, but not static correlation, and CASSCF captures static correlation but not dynamic correlation. Moreover, CASSCF indicates a closed shell for the singlet and two radical electrons for the triplet in the electrocyclic pathway (see Table 1 of the Supporting Information), implying that single Slater determinants are able to describe these structures, hence energetics from the single Kohn-Sham Slater determinant in DFT calculations are deemed reliable.

On the other hand, the converged NOON of singlet and triplet transition states and intermediates (Table 1 of the Supporting Information) of the sigmatropic routes, indicate that all sigmatropic transition states have some diradical character, and the sigmatropic stepwise intermediate is a pure diradical. DFT is unable to describe these more exotic electronic structures, indicating the necessity for CASPT2 calculations in order to get accurate energetics. ${ }^{81,82}$ However, CASSCF and CASPT2 energies were shown to be comparable for $[1,3]$ sigmatropic rearrangements of bicyclic and tricyclic vinylcyclobutanes, ${ }^{83}$ which are described by transition states highly similar in nature to the sigmatropic stepwise transition states in the present study, hence the levels of theory employed are considered to be sufficient. Moreover, CASSCF and M06$2 \mathrm{X}$ energies agree reasonably well for both sigmatropic pathways.

DFT calculations had accurately suggested that the stepwise sigmatropic pathway goes through open-shell transition states and a corresponding open-shell singlet biradical intermediate; however, they had incorrectly suggested the concerted sigmatropic pathway to proceed through a closed-shell transition state and therefore it seems to be an artifact of the M06-2X calculations to give rise to stable wave functions for the concerted closed-shell transition state, and therefore, no further calculations will be done on this transition state.

As a conclusion, the electrocyclic cascade is obviously preferred over the sigmatropic pathways; however, the activation barriers for the stepwise sigmatropic processes might be overcome at high temperatures. Moreover, DFT calculations suggested that the stepwise sigmatropic pathway goes through open-shell transition states and a corresponding open-shell singlet biradical intermediate and that the concerted sigmatropic pathway has a closed-shell transition state. NOON from CASSCF calculations on the other hand showed that all sigmatropic transition states have some diradical character, and the sigmatropic stepwise intermediate is a pure diradical.

B. Experimental Study. In order to experimentally investigate the possibility of a $[1,5]$ sigmatropic alkyl shift in a bicyclo[4.2.0] octa-2,4-diene system, the diol derivative 9 was synthesized in two steps from (E)-pent-2-en-4-yn-1-ol 11 (Scheme 6). Copper-mediated oxidative Glaser coupling and

Scheme 6. Synthesis of the Bicyclo[4.2.0] octa-2,4-diene diols 9 and $9-\mathrm{d}_{4}$
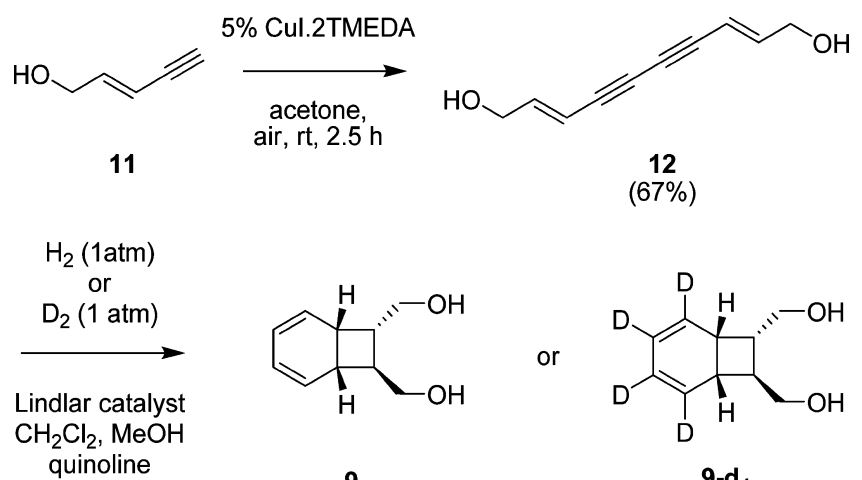

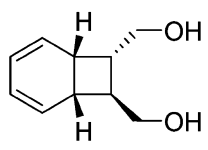

$(30-45 \%)$<smiles>[2H]C1=C([2H])[C@@H]2[C@H](CO)[C@H](CO)[C@@H]2C([2H])=C1[2H]</smiles>

9-d partial hydrogenation of the resulting symmetrical diyne diol 12 was followed in situ by a cascade of an $8 \pi$ - and a $6 \pi$ electrocyclic ring closures as previously described, ${ }^{2,13}$ giving the $4 \pi$ system 9 as the major product, in reasonable yield. When the reaction was run using deuterium gas $(99.8 \%$ atom $\mathrm{D})$, the expected diol $\mathbf{9}-\mathbf{d}_{\mathbf{4}}$ was obtained as a single isotopomer. This deuterium-labeled system was then used to study the thermal rearrangements.

The methyl esters of endiandric acids $\mathrm{D}$ and $\mathrm{E}$ are known to interconvert with a half-life of ca. $1.3 \mathrm{~h}$ at $70{ }^{\circ} \mathrm{C}$ in toluene. Thus, a similar equilibrium is expected to exist in the simpler diol 9, although the interconverting products are identical in this case. However, for the deuterium-labeled diol $\mathbf{9}-\mathbf{d}_{\mathbf{4}}$, this equilibrium would be unnoticed only if the rearrangement followed exclusively the electrocyclic ring opening pathway to the $6 \pi$ cyclooctatriene (COT) valence tautomer, which can 


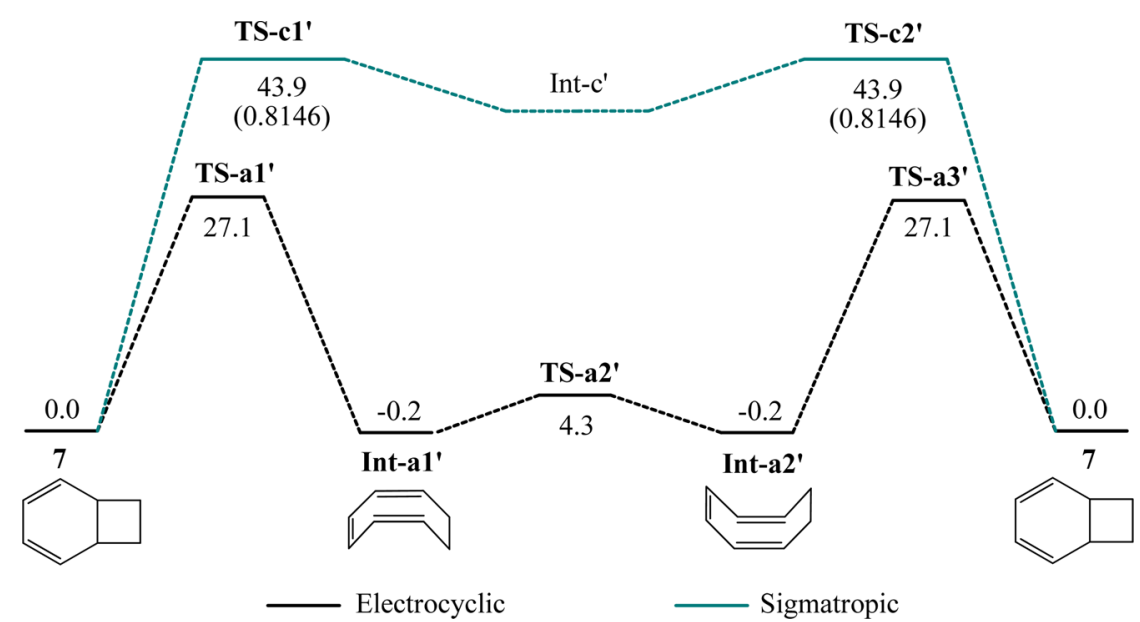

Figure 4. Free-energy profiles for the electrocyclic (M06-2X/6-31+G(d,p)) and sigmatropic pathways (UM06-2X/6-31+G(d,p)) and expectation values of the total spin $\left\langle S^{2}\right\rangle$ (in parentheses) in the thermal equilibration of bicyclo[4.2.0] octa-2,4-diene 7. For the sigmatropic pathway, no stable intermediate could be located, and IRC paths led to an unstable intermediate that disintegrated. All energies in kilocalories per mol.

then ring flip and close again, whereas the alternative single step pathway via a $[1,5]$ sigmatropic alkyl shift or walk rearrangement would result in extensive scrambling of the deuterium labels over the carbons of the six-membered ring (Scheme 5b). The expected isotopomers of diol $\mathbf{9}-\mathbf{d}_{\mathbf{4}}$ should be detected easily by the appearance of the diagnostic olefinic resonances in the proton NMR spectrum. However, when a solution of the diol $9-\mathrm{d}_{4}$ in toluene was heated at $110{ }^{\circ} \mathrm{C}$ for $1 \mathrm{~h}$, the starting material was recovered unchanged by NMR analysis. Consequently, at this temperature, the thermal equilibrium previously described by Nicolaou and Black for the endiandric acids does not constitute a walk rearrangement, as previously demonstrated also by computational results.

When dilute solutions of $\mathbf{9 - d _ { 4 }}$ were heated at temperatures between 170 and $195{ }^{\circ} \mathrm{C}$, olefinic resonances did appear in the proton NMR spectra, which were superimposable with those observed for the nonlabeled diol 9 both in $\mathrm{CDCl}_{3}$ and DMSO$d_{6}$. However, assignment to any of the six possible isotopomers was not possible via $1 \mathrm{D}$ or $2 \mathrm{D}$ NMR experiments. Rigorous chromatographic purification of the reaction mixture obtained after heating for $2 \mathrm{~h}$ at $190-195^{\circ} \mathrm{C}$ in acetonitrile (sealed tube) gave the unchanged diol $\mathbf{9}-\mathrm{d}_{\mathbf{4}}$ as a single isotopomer in about $30 \%$ yield. The observed olefinic resonances could therefore not be explained as D-scrambled products but must arise from other thermal reaction products. A similar complex mixture of products was obtained when the nonlabeled diol 9 was subjected to the same conditions, but none of the constituents could be fully identified. Finally, heating diols $\mathbf{9}$ and $\mathbf{9}-\mathbf{d}_{\mathbf{4}}$ at even higher temperatures (up to $230{ }^{\circ} \mathrm{C}$ ) in ethylene glycol (sealed tube) gave a very fast $(<10 \mathrm{~min})$ and complete consumption of the starting material, returning a rather complex and inseparable mixture of products. In contrast to most bicyclo[4.1.0]hepta-2,4-diene (norcaradiene) systems, the bicyclo[4.2.0] octa-2,4-diene system appears to have limited thermal stability. Furthermore, partial analysis by 2D NMR experiments seems to implicate the completely ring-opened acyclic tetraene valence tautomer as the parent structure for most of the observed thermal products, a reaction pathway, which is not available for the norcaradiene systems.

2. Thermal Equilibration of Bicyclo[4.2.0]octa-2,4diene 7 and Comparison with Literature. The electrocyclic and sigmatropic stepwise pathways were computationally explored for the parent bicyclo[4.2.0] octa-2,4-diene compound 7 (Figure 4) with the M06-2X/6-31+G(d,p) level of theory, in order to compare the thermal equilibration of this unsubstituted bicyclo[4.2.0] octa-2,4-diene with the norcaradiene system reported in literature.

The free-energy profiles for both pathways of the thermal equilibration of bicyclo[4.2.0] octa-2,4-diene 7 are shown in Figure 4. These look fairly similar to the free-energy profiles for bicyclo[4.2.0] octa-2,4-diene 9, again suggesting a clear preference for the electrocyclic cascade. While the bicyclo[4.2.0] octa-2,4-diene 9 was found to be more stable than its contorted cyclooctatriene intermediate, as anticipated in the introduction, this is not true for the bicyclo[4.2.0] octa2,4-diene 7 , which was found to be as stable as its contorted cyclooctatriene intermediate, as was also recently reported by Houk. ${ }^{55 a}$ Consequently, the sigmatropic pathway is shown to be less likely for bicyclo[4.2.0] octa-2,4-diene 7 when compared to the bicyclo[4.2.0] octa-2,4-diene 9. Furthermore, no stable singlet intermediate could be located for the unsubstituted bicyclo[4.2.0] octa-2,4-diene 7, whereas an open-shell singlet biradical intermediate was found for bicyclo[4.2.0] octa-2,4diene 9 , which can be attributed to the difference in stability for primary and secondary radicals. Nonetheless, the parent bicyclo[4.2.0] octa-2,4-diene compound 7 could undergo a "one step nonconcerted" sigmatropic shift, ${ }^{38,84}$ which proceeds without the formation of an intermediate.

The Gibbs free activation barrier for the electrocyclic route found here is very close to the ones reported earlier by Huisgen $^{42 \mathrm{~b}}$ and recently by Houk ${ }^{55 \mathrm{a}}\left(\Delta G^{\ddagger}=27.1 \pm 0.2 \mathrm{kcal} /\right.$ mol). Furthermore, although no stable singlet intermediate could be located for the parent bicyclo[4.2.0] octa-2,4-diene compound 7, appropriate substituents can favor the stepwise sigmatropic pathway, as demonstrated in the previous section for bicyclo[4.2.0] octa-2,4-diene 9. The calculated barriers for the sigmatropic bond cleavage $\left(\Delta G^{\ddagger}=43.9 \mathrm{kcal} / \mathrm{mol}\right)$ are within the range of experimental and predicted activation barriers for [1,5] alkyl shifts in bicyclo[4.1.0]hepta-2,4-dienes, which range from 35 to $45 \mathrm{kcal} / \mathrm{mol}^{38,39}$

As a conclusion, even though the electrocyclic cascade is more plausible, comparable barriers for the $[1,5]$ alkyl shifts of bicyclo[4.2.0] octa-2,4-dienes and bicyclo[4.1.0]hepta-2,4-dienes strongly suggest that the sigmatropic stepwise pathway 


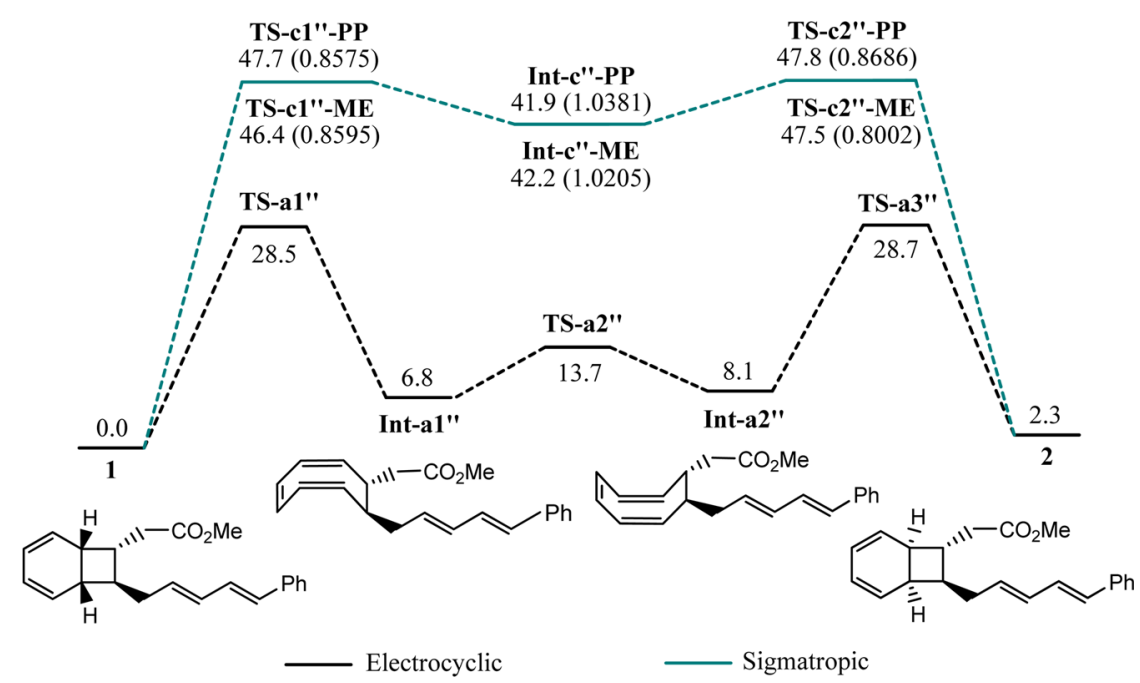

Figure 5. Free-energy profiles for the electrocyclic $(M 06-2 X / 6-31+\mathrm{G}(\mathrm{d}, \mathrm{p}))$ and sigmatropic pathways (UM06-2X/6-31+G(d,p)) and expectation values of the total spin $\left\langle S^{2}\right\rangle$ (in parentheses) in the thermal equilibration of endiandric acid methyl esters D/E (1/2). For the sigmatopic pathways, $\mathrm{PP}$ indicates the breaking of the $\mathrm{C}-\mathrm{C}$ bond close to the phenyl pentadienyl group and ME indicates the breaking of the $\mathrm{C}-\mathrm{C}$ bond close to the methyl ester group. All energies in kilocalories per mol.

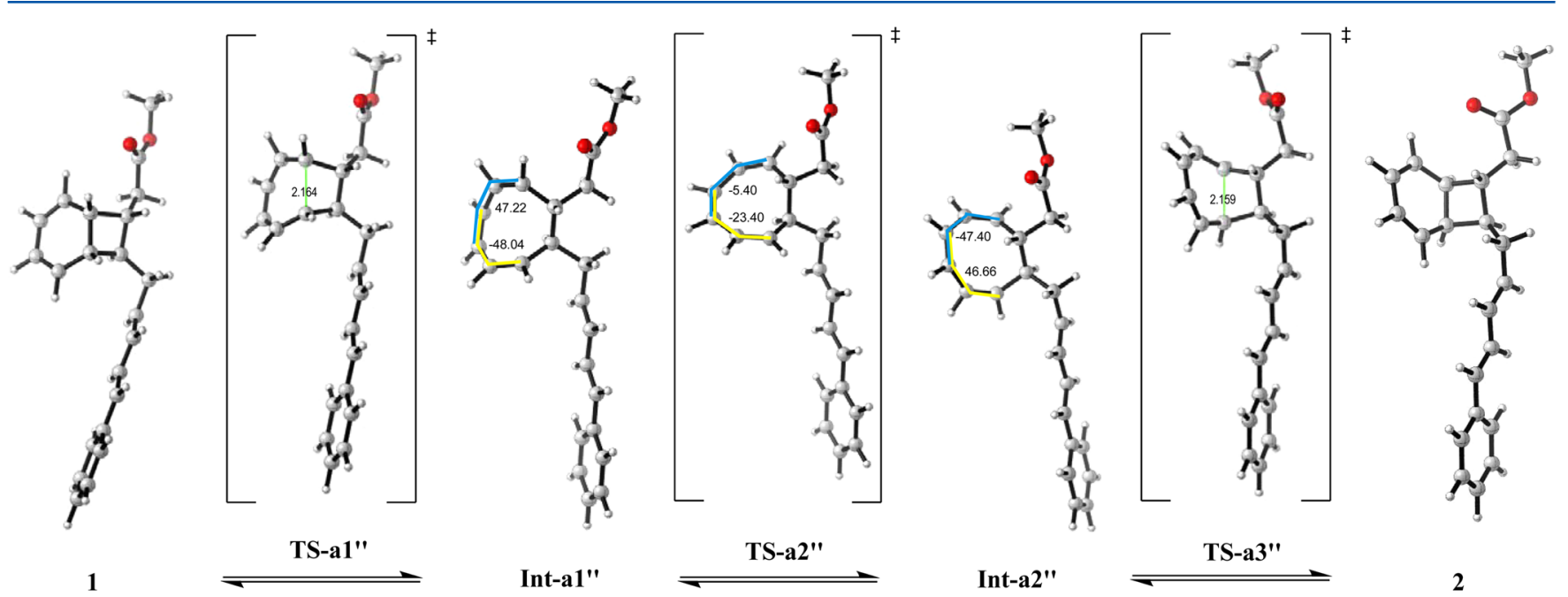

Figure 6. Electrocyclic cascade $(M 06-2 X / 6-31+G(d, p))$ for the thermal equilibration of endiandric acid methyl esters D/E (1/2). Some critical distances (green, angstroms) and dihedral angles (yellow and blue, in degrees) are shown.

is feasible at higher temperatures for appropriately substituted compounds.

3. Thermal Equilibration of Endiandric Acid Methyl Esters D/E (1/2). Finally, the electrocyclic and sigmatropic stepwise pathways were computationally explored for the thermal equilibration of endiandric acid methyl esters D/E (1/ 2). The free-energy profiles shown in Figure 5 reveal relative Gibbs free energies that are only slightly higher than those for the thermal equilibration of the diol derivate 9 and expectation values of total spin $\left\langle S^{2}\right\rangle$ that are comparable to those for the diol derivate 9, suggesting a clear preference for the electrocyclic cascade. Although the sigmatropic pathway might be feasible at high temperatures. The energetically favorable electrocyclic pathway along with optimized transition state and intermediate geometries is shown in Figure 6. A thorough conformational search was done on the phenyl pentadienyl group and the methyl ester group of methyl ester 1, and the most stable conformer is shown in Figure 6. All other transition states and intermediates originated from this conformation. Endiandric acid methyl esters 1/2 were found to be more stable with respect to their contorted cyclooctatriene (COT) intermediate, compared to the diol derivate 9, this favors the sigmatropic pathways. Moreover, substituents made it possible to locate a stable singlet intermediate for the stepwise sigmatropic pathway, whereas no stable intermediate could be located for the unsubstituted bicyclo[4.2.0] octa-2,4diene 7 . Therefore, the stepwise sigmatropic pathway may be plausible at higher temperatures for both the diol derivate 9 and the endiandric acid methyl esters $1 / 2$. Expectation values of total spin $\left\langle S^{2}\right\rangle$ from DFT calculations indicate that the sigmatropic pathway goes through open-shell transition states and a corresponding open-shell singlet biradical intermediate (Figure 5). Similarly, NOON from CASSCF calculations on the diol derivate 9 had shown that the sigmatropic transition states have some diradical character, and the sigmatropic intermediate is a pure diradical. It was also previously shown for the sigmatropic transition states of the diol derivate 9 that CASSCF and M06-2X energies are in very good agreement. Since the relative Gibbs free energies (M06-2X) for the thermal equilibration of methyl esters $\mathbf{1} / \mathbf{2}$ are comparable to those of 
the diol derivate 9 for all pathways, CASSCF calculations were not performed on the sigmatropic pathways of methyl esters $\mathbf{1}$ / 2, due to the large size of the system. However, this system is assumed to be comparable to the diol derivate 9, which was shown to proceed via singlet states for all pathways and have a sigmatropic pathway with diradical character.

\section{CONCLUSION}

The mechanism of thermal equilibration between endiandric acid methyl esters $\mathrm{D} / \mathrm{E}$ in particular and more generally the possibility of $[1,5]$ sigmatropic alkyl shifts (walk rearrangements) in bicyclo[4.2.0] octa-2,4-diene systems at high temperatures have been explored in a combined computational and experimental study, pointing to the following conclusions: (a) an electrocyclic cascade is clearly preferred over the sigmatropic pathways; the calculated free-energy barriers for this route, which was previously proposed by Nicolaou, are shown to be very close to the one for bicyclo[4.2.0] octa-2,4-diene reported by Huisgen. (b) The activation barriers for the sigmatropic process might be overcome at high temperatures. Calculated barriers for the sigmatropic stepwise pathway were shown to be comparable with the reaction barriers for the bicyclo[4.1.0]hepta-2,4-diene (norcaradiene) walk rearrangement. Nevertheless, this stepwise pathway is only feasible for appropriately substituted compounds. (c) DFT calculations suggested that the stepwise sigmatropic pathway goes through open-shell transition states and a corresponding open-shell singlet biradical intermediate, whereas a proposed concerted sigmatropic pathway has a closed-shell transition state. CASSCF calculations showed that all sigmatropic transition states have some diradical character, and the sigmatropic stepwise intermediate is a pure diradical. Therefore, the closed-shell concerted sigmatropic transition state TS-b that was located with DFT is not a true transition state.

Experimental NMR analysis on the thermal rearrangement of the deuterium-labeled diol $\left(\mathbf{9}-\mathbf{d}_{\mathbf{4}}\right)$, for which the electrocyclic and sigmatropic rearrangements would lead to different interconverting isotopomeric products, showed that in this model system, $[1,5]$ sigmatropic alkyl shifts do not occur with a significant reaction rate at temperatures up to $195{ }^{\circ} \mathrm{C}$. Higher temperatures could not be explored because of the limited thermal stability of this bicyclic system. Our results indicate that $[1,5]$ sigmatropic shifts should be energetically comparable processes both in bicyclo[4.2.0] octa-2,4-diene and bicyclo[4.1.0]hepta-2,4-diene compounds, but they have so far only been observed in the latter.

\section{EXPERIMENTAL SECTION}

General Methods. Reactions were monitored by thin layer chromatography (TLC) using UV254 precoated silicagel plates $(0.25$ $\mathrm{mm}$ thickness). The TLC plates were visualized using an anisaldehyde ( $5 \%$ anisaldehyde in ethanol with $1 \%$ sulfuric acid) or a PMA (5\% phosphomolybdic acid in ethanol) solution. Flash column chromatography was performed using silica gel $(0.063-0.200 \mathrm{~mm}$ particle size). ${ }^{1} \mathrm{H}$ NMR and ${ }^{13} \mathrm{C}$ NMR spectra were recorded on a $300 \mathrm{MHz}$ instrument at 300 and at $75 \mathrm{MHz}$, respectively. Chemical shifts $(\delta)$ are reported in units of parts per million (ppm), referenced relative to the residual ${ }^{1} \mathrm{H}$ or ${ }^{13} \mathrm{C}$ peaks of the used solvent as internal standards (chloroform- $d$ : $\delta_{\mathrm{H}} 7.26$ and $\delta_{\mathrm{C}} 77.16$; dimethyl sulfoxide- $d_{6}: \delta_{\mathrm{H}} 2.49$ and $\left.\delta_{\mathrm{C}} 39.50\right)$. The following abbreviations were used to explain the multiplicities: s, singlet; $\mathrm{d}$, doublet; $\mathrm{t}$, triplet; $\mathrm{q}$, quadruplet; $\mathrm{m}$, multiplet; br, broadened; band, several overlapping signals; $A B, A B$ system with strongly skewed signals. Where given and if appropriate, assignments of resonances were confirmed by standard COSY (GPQF) and HSQC(EDETGP) 2D NMR experiments. Infrared spectra (IR) were recorded on a FTIR spectrometer and reported in wave numbers $\left(\mathrm{cm}^{-1}\right)$. Samples were prepared as a thin film (neat) on the $\mathrm{KBr}$ plate. Mass spectra (MS) were recorded on an ESI-single quadrupole detector type VL. High-Resolution Mass Spectra (HRMS) were recorded on an accurate-mass quadrupole time-of-flight mass spectrometer. Reported melting point ranges were determined after iterative crystallization until a stable value was obtained.

Materials. All chemicals and solvents were purchased and used without any further purification, except dichloromethane, which was distilled from $\mathrm{CaH}_{2}$ prior to use. (E)-pent-2-en-4-yn-1-ol 11 is a commercially available compound but is prohibitively expensive and not readily available from standard suppliers. However, it was easily prepared in two steps from ethynyl trimethylsilane.

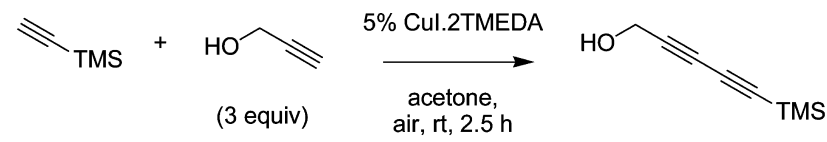

$58 \%$

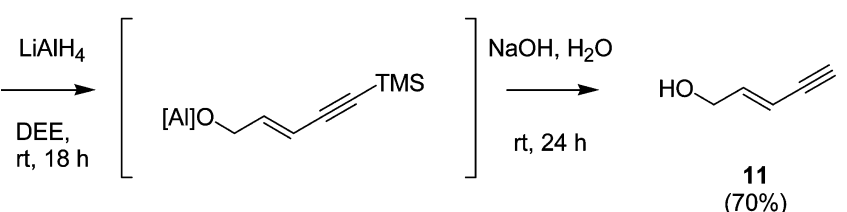

(E)-Pent-2-en-4-yn-1-ol 11. A solution of 2-propyn-1-ol (463 mg, $8.3 \mathrm{mmol})$ and ethynyl trimethylsilane $(50 \mathrm{mg}, 0.51 \mathrm{mmol})$ in acetone $(15 \mathrm{~mL})$ was added to a vigorously stirred solution of copper(I)iodide $(169 \mathrm{mg}, 0.89 \mathrm{mmol})$ and tetramethylethylenediamine $(207 \mathrm{mg}, 1.78$ $\mathrm{mmol})$ in acetone $(20 \mathrm{~mL})$, in a reaction flask that was open to air. The resulting mixture was stirred open to air for $5 \mathrm{~min}$, and then a solution of ethynyl trimethylsilane $(450 \mathrm{mg}, 4.58 \mathrm{mmol})$ and prop-2yn-1-ol $(250 \mathrm{mg}, 4.5 \mathrm{mmol})$ in acetone $(15 \mathrm{~mL})$ was added dropwise over $20 \mathrm{~min}$. The reaction was stirred for another $2 \mathrm{~h}$, the bulk of the acetone was removed under reduced pressure, and the residue was diluted with methyl-tert-butylether $(100 \mathrm{~mL})$. The organic layer was washed with a saturated aqueous solution of ammonium chloride $(3 \times$ $10 \mathrm{~mL})$, water $(10 \mathrm{~mL})$, and brine $(10 \mathrm{~mL})$, dried over magnesium sulfate, and concentrated in vacuo. The residue was purified by chromatography on silica, eluting with $30 \%$ methyl-tert-butylether in light petroleum (bp $40-60{ }^{\circ} \mathrm{C}$ ), to give 5-trimethylsilanyl-penta-2,4diyn-1-ol (450 mg, 58\%) as a clear colorless liquid.

A solution of the alcohol obtained above in diethyl ether $(7.0 \mathrm{~mL})$ was added dropwise over $5 \mathrm{~min}$ to a suspension of lithium aluminumhydride $(333 \mathrm{mg}, 8.87 \mathrm{mmol})$ in diethyl ether $(7.0 \mathrm{~mL})$ that was vigorously stirred at $0{ }^{\circ} \mathrm{C}$. The resulting mixture was warmed to room temperature and stirred for $18 \mathrm{~h}$. Then, the reaction mixture was cooled to $0{ }^{\circ} \mathrm{C}$, and water $(0.33 \mathrm{~mL})$ was added carefully, followed by a $15 \%$ aqueous solution of sodium hydroxide $(0.33 \mathrm{~mL})$ and water $(1.0 \mathrm{~mL})$. The resulting white suspension was stirred vigorously for 24 $\mathrm{h}$, and then filtered over a pad of silica, which was thouroughly washed with methyl-tert-butylether. The filtrate was concentrated to give $(E)$ pent-2-en-4-yn-1-ol 11 (170 mg, 70\%) as a volatile, clear colorless liquid which contained residual trimethyl silanol and solvent but was used in the next step without further purification. The compound showed proton NMR data that were consistent with data reported for this compound, previously synthesized using different methods. ${ }^{85-88}$

(2E,8E)-Deca-2,8-diene-4,6-diyne-1,10-diol 12. The crude alcohol $11(170 \mathrm{mg})$ was dissolved in acetone $(3 \mathrm{~mL})$ and then added over 5 min to a solution of copper(I)iodide $(28 \mathrm{mg}, 0.15 \mathrm{mmol})$ and tetramethylethylenediamine $(35 \mathrm{mg}, 0.30 \mathrm{mmol})$ in acetone $(3 \mathrm{~mL})$ that was stirred open to air at room temperature. After stirring for another $2 \mathrm{~h}$, the bulk of the acetone was removed under reduced pressure, and the dark residue $(\sim 0.5 \mathrm{~mL})$ was directly subjected to chromatography over silica, eluting with a $3: 1$ mixture of methyl-tertbutylether and light petroleum (bp $\left.40-60{ }^{\circ} \mathrm{C}\right)$, to give $(2 E, 8 E)$-deca2,8-diene-4,6-diyne-1,10-diol 12 (114 mg, 67\%) as an off-white solid. 
Mp $152-153{ }^{\circ} \mathrm{C}$ (recryst. from methyl-tert-butylether and light petroleum). IR $\nu_{\max }: 3284(\mathrm{~s}), 2894,2207(\mathrm{vw}) .{ }^{1} \mathrm{H}$ NMR $(300 \mathrm{MHz}$, DMSO- $\left.d_{6}\right): \delta 3.18\left(4 \mathrm{H}\right.$, ddd, $J=5.4,4.3$, and $\left.1.9 \mathrm{~Hz}, 2 \times \mathrm{CH}_{2} \mathrm{OH}\right)$, $4.17(2 \mathrm{H}, \mathrm{t}, J=5.4 \mathrm{~Hz}, 2 \times \mathrm{OH}), 4.98(2 \mathrm{H}, \mathrm{dt}, J=15.6$ and $1.9 \mathrm{~Hz}, 2$ $\left.\times \mathrm{CH}=\mathrm{CHCH}_{2}\right), 5.60(2 \mathrm{H}, \mathrm{dt}, J=15.6$ and $4.3 \mathrm{~Hz}, 2 \times \mathrm{CH}=$ $\left.\mathrm{CHCH}_{2}\right) \cdot{ }^{13} \mathrm{C}$ NMR $\left(75 \mathrm{MHz}, \mathrm{DMSO}-d_{6}\right): \delta 60.8\left(2 \mathrm{CH}_{2}\right), 73.5(2 \mathrm{C})$, $80.3(2 \mathrm{C}), 106.1(2 \mathrm{CH}), 149.1(2 \mathrm{CH})$. MS(ESI): $m / z 145.1\left(\mathrm{MH}^{+}-\right.$ $\left.\mathrm{H}_{2} \mathrm{O}\right)$. HRMS (ESI) calcd. For $\mathrm{C}_{10} \mathrm{H}_{9} \mathrm{O}\left(\mathrm{m} / z \mathrm{M}+\mathrm{H}^{+}-\mathrm{H}_{2} \mathrm{O}\right)$, 145.0648; found, 145.0643 and calcd. For $\mathrm{C}_{10} \mathrm{H}_{11} \mathrm{O}_{2}(\mathrm{~m} / z \mathrm{M}-\mathrm{H}+$, negative mode), 161.0608; found, 161.0603 .

(8-Hydroxymethyl-bicyclo[4.2.0]octa-2,4-dien-7-yl)-methanol 9. Lindlar's catalyst (palladium, $5 \%$ on calcium carbonate, poisoned with lead (purchased from Aldrich chemical company), $102 \mathrm{mg}$ ) was added to a solution of diol $12(50.0 \mathrm{mg}, 0.308 \mathrm{mmol})$ and quinoline $(0.050 \mathrm{~mL})$ in dichloromethane $(9.0 \mathrm{~mL})$ and methanol $(1.0 \mathrm{~mL})$. The resulting suspension was degassed and placed under an atmosphere of hydrogen gas. The reaction progress was closely monitored by thin layer chromatography. The starting material was usually quickly converted into the monohydrogenated product (5-10 $\mathrm{min}$ ), which was then slowly transformed into a number of products, but mainly the diol $9(0.5-4 \mathrm{~h})$. The reaction mixture was degassed upon consumption of the monohydrogenated intermediate (as judged by TLC) and filtered over a short pad of silica, which was washed with methyl-tert-butylether. The filtrate was concentrated in vacuo, and the residue was purified by chromatography over silica, eluting with $2.5 \%$ methanol in chloroform. The obtained product was further purified by chromatography over silica, eluting with a 2:1 mixture of methyl-tertbutylether and light petroleum (bp $40-60^{\circ} \mathrm{C}$ ). This afforded the pure diol $12(23.5 \mathrm{mg}, 45 \%)$ as a very viscous, clear colorless oil [in some runs, lower yields were obtained (down to $30 \%)$ ]. IR $\nu_{\max }: 3318(\mathrm{~s})$, 2922(s), 1461, 1376, 1028. ${ }^{1} \mathrm{H}$ NMR $\left(300 \mathrm{MHz}, \mathrm{CDCl}_{3}\right): \delta 2.67-2.74$ $(3 \mathrm{H}$, band, $3 \times$ cyclobutane $-\mathrm{CH}), 3.13-3.20(1 \mathrm{H}, \mathrm{m},=\mathrm{CH}-$ CHcyclobutane), $3.45-3.51(1 \mathrm{H}, \mathrm{m}, \mathrm{CHHOH}), 3.76(1 \mathrm{H}, \mathrm{dd}, J=$ 10.2 and $3.6 \mathrm{~Hz}, \mathrm{CHHOH}), 3.79-3.85\left(2 \mathrm{H}, \mathrm{m}, \mathrm{CH}_{2} \mathrm{OH}\right), 5.53(1 \mathrm{H}$, $\mathrm{dd}(\mathrm{br}), J=9.7$ and $3.8 \mathrm{~Hz}, \mathrm{CH}=\mathrm{CH}-\mathrm{CH}=\mathrm{CH}), 5.60(1 \mathrm{H}, \mathrm{dd}(\mathrm{br})$, $J=9.5$ and $3.9 \mathrm{~Hz}, \mathrm{CH}=\mathrm{CH}-\mathrm{CH}=\mathrm{CH}), 5.72(1 \mathrm{H}, \mathrm{dd}, J=9.5$ and $5.5 \mathrm{~Hz}, \mathrm{CH}=\mathrm{CH}-\mathrm{CH}=\mathrm{CH}), 5.87(1 \mathrm{H}, \operatorname{ddd}(\mathrm{br}), J=9.7,5.5$, and $1.7 \mathrm{~Hz}, \mathrm{CH}=\mathrm{CH}-\mathrm{CH}=\mathrm{CH}) .{ }^{13} \mathrm{C}$ NMR $\left(75 \mathrm{MHz}, \mathrm{CDCl}_{3}\right): \delta 32.5$ $(\mathrm{CH}), 33.0(\mathrm{CH}), 51.1(\mathrm{CH}), 52.5(\mathrm{CH}), 62.8\left(\mathrm{CH}_{2}\right), 65.5\left(\mathrm{CH}_{2}\right)$, $122.3(\mathrm{CH}), 124.3(\mathrm{CH}), 125.5(\mathrm{CH}), 126.1(\mathrm{CH}) .{ }^{1} \mathrm{H}$ NMR $(300$ $\left.\mathrm{MHz}, \mathrm{DMSO}-d_{6}\right): \delta 2.25\left(1 \mathrm{H}\right.$, app. quintet, $\left.J=\sim 7.2 \mathrm{~Hz}, \mathrm{C}^{\prime}-H\right), 2.59$ $\left(1 \mathrm{H}\right.$, (app. q)d, $J=\sim 8.5$ and $\left.6.9 \mathrm{~Hz}, \mathrm{C}^{\prime}-\mathrm{H}\right), 2.66(1 \mathrm{H}, \mathrm{ddd}, J=11.1$, 8.1, and $\left.5.3 \mathrm{~Hz}, \mathrm{C} 6^{\prime}-H\right), 3.02\left(1 \mathrm{H}\right.$, app.t(br), $\left.J=\sim 9.5 \mathrm{~Hz}, \mathrm{Cl}^{\prime}-\mathrm{H}\right)$, $3.34\left(2 \mathrm{H}\right.$, app.t, $\left.\mathrm{J}=\sim 5.7 \mathrm{~Hz}, \mathrm{C} 1-\mathrm{H}_{2} \mathrm{OH}\right), 3.48(1 \mathrm{H}, \mathrm{d}(\mathrm{AB}) \mathrm{dd}, J=$ 10.4, 6.7, and 4.5 Hz, C1"- $\mathrm{HHOH}), 3.55(1 \mathrm{H}, \mathrm{d}(\mathrm{AB}) \mathrm{dd}, J=10.4,8.7$, and $\left.5.3 \mathrm{~Hz}, \mathrm{Cl}^{\prime \prime}-\mathrm{HHOH}\right), 4.35\left(1 \mathrm{H}\right.$, app.t, $\left.J=\sim 4.9 \mathrm{~Hz}, \mathrm{Cl}^{\prime \prime}-\mathrm{H}_{2} \mathrm{OH}\right)$, $4.48\left(1 \mathrm{H}\right.$, app.t, $\left.J=\sim 5.3 \mathrm{~Hz}, \mathrm{C} 1-\mathrm{H}_{2} \mathrm{OH}\right), 5.55(1 \mathrm{H}, \mathrm{d}(\mathrm{AB}) \mathrm{d}, J=9.7$ and $\left.4.4 \mathrm{~Hz}, \mathrm{CS}^{\prime}-\mathrm{H}\right), 5.56-5.61\left(1 \mathrm{H}, \mathrm{m}, \mathrm{C}^{\prime}-\mathrm{H}\right), 5.62(1 \mathrm{H}, \mathrm{d}(\mathrm{AB}) \mathrm{d}, J$ $=9.7$ and $\left.4.9 \mathrm{~Hz}, \mathrm{C} 4^{\prime}-\mathrm{H}\right), 5.80(1 \mathrm{H}$, dd(app.t), $J=9.9,4.9$, and $1.5 \mathrm{~Hz}$, $\left.\mathrm{C} 3{ }^{\prime}-H\right) .{ }^{13} \mathrm{C}$ NMR $\left(75 \mathrm{MHz}, \mathrm{DMSO}-d_{6}\right): \delta 32.1(\mathrm{CH}), 32.2(\mathrm{CH})$, $48.4(\mathrm{CH}), 51.3(\mathrm{CH}), 61.2\left(\mathrm{CH}_{2}\right), 63.5\left(\mathrm{CH}_{2}\right), 121.3(\mathrm{CH}), 123.5$ $(\mathrm{CH}), 126.1(\mathrm{CH}), 127.2(\mathrm{CH}) . \mathrm{MS}(\mathrm{ESI}): m / z 167.1\left(\mathrm{M}+\mathrm{H}^{+}, 26\right)$, $149.1\left(\mathrm{M}+\mathrm{H}^{+}-\mathrm{H}_{2} \mathrm{O}, 100\right)$. HRMS (ESI): calcd. For $\mathrm{C}_{10} \mathrm{H}_{15} \mathrm{O}_{2}(\mathrm{~m} / z \mathrm{M}$ $\left.+\mathrm{H}^{+}\right)$, 167.1067; found, 167.1066 .

$\left[2,3,4,5-{ }^{2} \mathrm{H}_{4}\right]$-(8-Hydroxymethyl-bicyclo[4.2.0]octa-2,4-dien-7-yl)methanol 9- $d_{4}$. The general procedure for the synthesis of bicyclic diol 9 was followed, but deuterium gas $(99.8 \%$ atom D) was used instead of hydrogen gas. Starting from $50 \mathrm{mg}$ of diol $12(0.295 \mathrm{mmol})$, chromatography as described for compound 9 gave the deuteriumlabeled bicyclic diol $\mathbf{9 - \mathbf { d } _ { 4 }}(18.0 \mathrm{mg}, 34 \%)$ as a clear viscous oil. IR $\nu_{\max }$ : 3336(s), 2922(s), 1462, 1376, 1025. ${ }^{1} \mathrm{H}$ NMR $\left(300 \mathrm{MHz}, \mathrm{CDCl}_{3}\right): \delta$ 2.67-2.74 (3H, band, $3 \times \mathrm{CH}), 3.14-3.19(1 \mathrm{H}, \mathrm{m},=\mathrm{CD}-\mathrm{CH})$, $3.45-3.51(1 \mathrm{H}, \mathrm{m}, \mathrm{CHHOH}), 3.76(1 \mathrm{H}, \mathrm{dd}, J=10.2$ and $3.6 \mathrm{~Hz}$, $\mathrm{CHHOH}), 3.79-3.85\left(2 \mathrm{H}, \mathrm{m}, \mathrm{CH}_{2} \mathrm{OH}\right) .{ }^{13} \mathrm{C} \mathrm{NMR}(75 \mathrm{MHz}$, $\left.\mathrm{CDCl}_{3}\right): \delta 32.5(\mathrm{CH}), 33.0(\mathrm{CH}), 51.1(\mathrm{CH}), 52.5(\mathrm{CH}), 62.8\left(\mathrm{CH}_{2}\right)$, $65.5\left(\mathrm{CH}_{2}\right)$. MS(ESI): $m / z 171.1\left(\mathrm{MH}^{+}, 58\right), 153.1\left(\mathrm{MH}^{+}-\mathrm{H}_{2} \mathrm{O}\right.$, 100). HRMS (ESI): calcd. For $\mathrm{C}_{10} \mathrm{D}_{4} \mathrm{H}_{11} \mathrm{O}_{2}\left(\mathrm{~m} / z \mathrm{M}+\mathrm{H}^{+}\right), 171.1318$, found, 171.1323 .

Thermal Equilibration Experiments. A solution of the diol 9 or the diol $9-\mathrm{d}_{4}$ ( 2 to $5 \mathrm{mg}$ per run) in acetonitrile $(2.0 \mathrm{~mL})$ was neutralized with $\sim 1 \mathrm{mg}$ of sodium bicarbonate and purged with argon. The solution was then stirred in a closed reaction vessel under microwave heating (CEM Discover). The reaction temperature and vessel pressure were monitored by external surface sensors. Reactions in acetonitrile were maintained at temperatures between either 170-175 ${ }^{\circ} \mathrm{C}$ or $190-195^{\circ} \mathrm{C}$ for $1-6 \mathrm{~h}$ [which was the highest temperature that could be achieved in this solvent $\left.\left(p_{\max }=17.0 \mathrm{bar}\right)\right]$. Reactions were monitored by TLC and NMR, and the reaction mixtures were increasingly complex with reaction temperature and time. The starting materials were isolated unchanged from the reaction mixtures by careful chromatography over silica, eluting with $2 \%$ methanol in chloroform. The obtained products $(0.5-2.5 \mathrm{mg}, 25-50 \%)$ showed ${ }^{1} \mathrm{H}$ NMR spectra which were indistinguishable from those of the starting materials 9 or $9-d_{4}$. The same experiments performed in ethylene glycol, which allows reaction temperatures higher than 200 ${ }^{\circ} \mathrm{C}$, gave similar results. However, no trace of starting material remained after heating to $230{ }^{\circ} \mathrm{C}(20 \mathrm{~min})$ in these experiments, as judged by TLC and NMR.

\section{ASSOCIATED CONTENT}

\section{Supporting Information}

Cartesian coordinates and energies of M06-2X/6-31+G(d,p) optimized geometries, imaginary and low frequencies of transition states. Full reference 59. Natural orbital occupation numbers (NOON) of transition states and intermediates for the thermal rearrangement of $9(\operatorname{CASSCF}(6,6) / 6-31+G(d, p) / /$ UM06-2X/6-31+G(d,p)) and discussion. NMR spectra for compounds $\mathbf{9}, \mathbf{9 - d _ { 4 }}, \mathbf{1 1}, \mathbf{1 2}$. This material is available free of charge via the Internet at http://pubs.acs.org.

\section{AUTHOR INFORMATION}

\section{Corresponding Author}

*E-mail: saron.catak@boun.edu.tr.

\section{Notes}

The authors declare no competing financial interest.

\section{ACKNOWLEDGMENTS}

The Fund for Scientific Research Flanders (FWO) and the Research Board of Ghent University are acknowledged for financial support. L.H. thanks the Spanish MICIIN (MAT201129174-C02-02) for financial support. Computational resources and services used in this work were provided by Ghent University.

\section{REFERENCES}

(1) Bandaranayake, W. M.; Banfield, J. E.; Black, D. S. C.; Fallon, G. D.; Gatehouse, B. M. Chem. Commun. 1980, 162.

(2) Bandaranayake, W. M.; Banfield, J. E.; Black, D. S. C. Chem. Commun. 1980, 902.

(3) Azmi, M. N.; Gény, C.; Leverrier, A.; Litaudon, M.; Dumontet, V.; Birlirakis, N.; Guéritte, F.; Leong, K. H.; Halim, S. N. A.; Mohamad, K.; Awang, K. Molecules 2014, 19, 1732.

(4) Chouna, J. R.; Nkeng-Efouet, P. A.; Lenta, B. N.; Devkota, K. P.; Neumann, B.; Stammler, H.-G.; Kimbu, S. F.; Sewald, N. Phytochemistry 2009, 70, 684.

(5) Chouna, J. R.; Nkeng-Efouet, P. A.; Lenta, B. N.; Wansi, J. D.; Kimbu, S. F.; Sewald. Phytochem. Lett. 2010, 3, 13.

(6) Talontsi, F. M.; Lamshöft, M.; Bauer, J. O.; Razakarivony, A. A.; Andriamihaja, B.; Strohmann, C.; Spiteller, M. J. Nat. Prod. 2013, 76, 97.

(7) Yang, P.-S.; Cheng, M.-J.; Peng, C.-F.; Chen, J.-J.; Chen, I.-S. J. Nat. Prod. 2009, 72, 53.

(8) Williams, R. B.; Martin, S. M.; Hu, J.-F.; Norman, V. L.; Goering, M. G.; Loss, S.; O’Neil-Johnson, M.; Eldridge, G. R.; Starks, C. M. J. Nat. Prod. 2012, 75, 1319. 
(9) Nicolaou, K. C.; Petasis, N. A.; Zipkin, R. E.; Uenishi, J. J. Am. Chem. Soc. 1982, 104, 5555.

(10) Nicolaou, K. C.; Petasis, N. A.; Uenishi, J.; Zipkin, R. E. J. Am. Chem. Soc. 1982, 104, 5557.

(11) Nicolaou, K. C.; Zipkin, R. E.; Petasis, N. A. J. Am. Chem. Soc. 1982, 104, 5558.

(12) Nicolaou, K. C.; Petasis, N. A.; Zipkin, R. E. J. Am. Chem. Soc. 1982, 104, 5560 .

(13) Nicolaou, K. C.; Chen, J. S. Chem. Soc. Rev. 2009, 38, 2993.

(14) A notable exception is the well-known $[1,5]$ hydrogen shift of cyclopentadienes, which is a fast reaction at room temperature, for computational studies see: Hess, B. A.; Baldwin, J. E. J. Org. Chem. 2002, 67, 6025 .

(15) Saettel, N. J.; Wiest, O. J. Org. Chem. 2000, 65, 2331.

(16) Alabugin, I. V.; Manoharan, M.; Breiner, B.; Lewis, F. D. J. Am. Chem. Soc. 2003, 125, 9329 and references therein.

(17) Tantillo, D. J.; Lee, J. K. Annu. Rep. Prog. Chem., Sect. B: Org. Chem. 2007, 103, 272 and refs therein.

(18) Beaudry, C. M.; Malerich, J. P.; Trauner, D. Chem. Rev. 2005, $105,4757$.

(19) Houk, K. N.; Gonzalez, J.; Li, Y. Acc. Chem. Res. 1995, $28,81$.

(20) Houk, K. N.; Li, Y.; Evanseck, J. D. Angew. Chem., Int. Ed. Engl. 1992, 31, 682 .

(21) Woodward, R. B.; Hoffmann, R. J. Am. Chem. Soc. 1965, 87, 2511.

(22) Hoffmann, R; Woodward, R. B. Acc. Chem. Res. 1968, 1, 17.

(23) Woodward, R. B.; Hoffmann, R. Angew. Chem., Int. Ed. Engl. 1969, 8, 781.

(24) Woodward, R. B.; Hoffmann, R. J. Am. Chem. Soc. 1965, 87, 395.

(25) Newman-Evans, R. H.; Simon, R. J.; Carpenter, B. K. J. Org. Chem. 1990, 55, 695.

(26) Carpenter, B. K. Angew. Chem., Int. Ed. 1998, 37, 3340.

(27) Reyes, M. B.; Lobbovsky, E. B.; Carpenter, B. K. J. Am. Chem. Soc. 2002, 124, 641 and references therein.

(28) Carpenter, B. K. J. Am. Chem. Soc. 1995, 117, 6336.

(29) Boersma, M. A. M.; De Haan, J. W.; Kloosterziel, H.; Van de

Ven, L. J. M. J. Chem. Soc., Chem. Commun. 1970, 1168.

(30) Jensen, F. J. Am. Chem. Soc. 1989, 111, 4643.

(31) Klärner, F. G. Top. Stereochem. 1984, 15, 1.

(32) Klärner, F. G. In Topics in Stereochemistry; John Wiley \& Sons, Inc.: Hoboken, NJ, 2007; p 1.

(33) Bulo, R. E.; Jansen, H.; Ehlers, A. W.; de Kanter, F. J. J.; Schakel, M.; Lutz, M.; Spek, A. L.; Lammertsma, K. Angew. Chem., Int. Ed. 2004, 43, 714.

(34) Bulo, R. E.; Allaart, F.; Ehlers, A. W.; de Kanter, F. J. J.; Schakel, M.; Lutz, M.; Spek, A. L.; Lammertsma, K. J. Am. Chem. Soc. 2006, $128,12169$.

(35) Jensen, F. J. Am. Chem. Soc. 1989, 111, 4643.

(36) Klärner, F. G.; Wette, M. Chem. Ber. 1978, 111, 282.

(37) Berson, J. A.; Willcott, M. R. J. Am. Chem. Soc. 1966, 88, 2494.

(38) Kless, A.; Nendel, M.; Wilsey, S.; Houk, K. N. J. Am. Chem. Soc. 1999, 121, 4524 and refs therein.

(39) Jarzecki, A. A.; Gajewski, J.; Davidson, E. R. J. Am. Chem. Soc. 1999, 121, 6928.

(40) Klärner, F. G.; Brassel, B. J. Am. Chem. Soc. 1980, 102, 2469.

(41) Baldwin, J. E.; Broline, B. M. J. Am. Chem. Soc. 1982, 104, 2857.

(42) This equilibrium is mainly governed by substitution at C7 and C8 positions, see: (a) Huisgen, R.; Dahmen, A.; Huber, H. J. Am. Chem. Soc. 1967, 89, 7130. (b) Huisgen, R.; Boche, G.; Dahmen, A.; Hechtl, W. Tetrahedron Lett. 1968, 5215. (c) Fry, A. J. Tetrahedron 2008, 64, 2101.

(43) Leber, P. A.; Baldwin, J. E. Acc. Chem. Res. 2002, 35, 279.

(44) Baldwin, J. E. Chem. Rev. 2003, 103, 1197.

(45) Baldwin, J. E.; Leber, P. A. Org. Biomol. Chem. 2008, 6, 36.

(46) Hudlicky, T.; Reed, J. Angew. Chem., Int. Ed. 2010, 49, 4864.

(47) Bulo, R. E.; Ehlers, A. W.; Grimme, S.; Lammertsma, K. J. Am. Chem. Soc. 2002, 124, 13903.

(48) Hehre, W. J.; Ditchfield, R.; Pople, J. A. J. Chem. Phys. 1972, 56, 2257.
(49) Krishnan, R.; Binkley, J. S.; Seeger, R.; Pople, J. A. J. Chem. Phys. 1980, 72, 650.

(50) Becke, A. D. J. Chem. Phys. 1993, 98, 5648.

(51) Lee, C.; Yang, W.; Parr, R. G. Phys. Rev. B 1988, 37, 785.

(52) Zhao, Y.; Truhlar, D. G. Acc. Chem. Res. 2008, 41, 157.

(53) Zhao, Y.; Truhlar, D. Theor. Chem. Acc. 2008, 120, 215.

(54) Grimme, S.; Antony, J.; Ehrlich, S.; Krieg, S. J. Chem. Phys. 2010, 132, 154104.

(55) For literature precedents for the successful use of B3LYP and M06-2X calculations on (related) electrocyclic and sigmatropic reactions, we refer the reader to ref 39 and (a) Patel, A.; Houk, K. N. J. Org. Chem. 2014, 79, 11370. (b) Wang, X.-N.; Krenske, E. H.; Johnston, R. C.; Houk, K. N.; Hsung, R. P. J. Am. Chem. Soc. 2014, 136, 9802. (c) Leach, A. G.; Catak, S.; Houk, K. N. Chem.-Eur. J. 2002, 8, 1290.

(56) Fukui, K. Acc. Chem. Res. 1981, 14, 363.

(57) Hratchian, H. P.; Schlegel, H. B.; Clifford, E. D.; Frenking, G.; Kwang, S. K.; Gustavo, E. S. In Theory and Applications of Computational Chemistry; Elsevier: Amsterdam, 2005; p 195.

(58) Seeger, R.; Pople, J. A. J. Chem. Phys. 1977, 66, 3045.

(59) Bauernschmitt, R.; Ahlrichs, R. J. Chem. Phys. 1996, 104, 9047.

(60) Frisch, M. J. et al., Gaussian 09, , revision A.02; Gaussian, Inc.: Wallingford, CT, 2009.

(61) For literature precedents for the successful use of CASSCF calculations on related electrocyclic and sigmatropic reactions, we refer the reader to ref 27, ref 38 and (a) Gutierrez, O.; Harrison, J. G.; Pemberton, R. P.; Dean, J. T. Chem.-Eur. J. 2012, 18, 11029.

(b) Tao, H.-R.; Fang, D.-C. Theor. Chem. Acc. 2008, 121, 91.

(62) White, S. R.; Martin, R. L. J. Chem. Phys. 1999, 110, 4127.

(63) Chan, G. K.-L.; Head-Gordon, M. J. Chem. Phys. 2002, 116, 4462.

(64) Wouters, S.; Bogaerts, T.; Van Der Voort, P.; Van Speybroeck, V.; Van Neck, D. J. Chem. Phys. 2014, 140, 241103.

(65) Wouters, S.; Poelmans, W.; Ayers, P. W.; Van Neck, D. Comput. Phys. Commun. 2014, 185, 1501.

(66) Wouters, S.; Van Neck, D. Eur. Phys. J. D 2014, 68, 272

(67) Wiest, O.; Montiel, D. C.; Houk, K. N. J. Phys. Chem. A 1997, $101,8378$.

(68) Hrovat, D. A.; Borden, W. T. J. Am. Chem. Soc. 2001, 123, 4069.

(69) Doering, W. v. E.; Ekmanis, J. L.; Belfield, K. D.; Klärner, F. G.; Krawczyk, B. J. Am. Chem. Soc. 2001, 123, 5532.

(70) Guner, V.; Khuong, K. S.; Leach, A. G.; Lee, P. S.; Bartberger, M. D.; Houk, K. N. J. Phys. Chem. A 2003, 107, 11445.

(71) Rodríguez-Otero, J.; Cabaleiro-Lago, E. M.; Peña-Gallego, Á. Tetrahedron 2007, 63, 2191.

(72) Jursic, B. S. Comp. Theor. Chem. 1995, 358, 139.

(73) Jursic, B.; Zdravkovski, Z. J. Chem. Soc., Perkin Trans. 2 1995, 1223.

(74) Goldstein, E.; Beno, B.; Houk, K. N. J. Am. Chem. Soc. 1996, 118,6036 and references therein.

(75) Beno, B. R.; Wilsey, S.; Houk, K. N. J. Am. Chem. Soc. 1999, 121, 4816.

(76) Houk, K. N.; Beno, B. R.; Nendel, M.; Black, K.; Yoo, H. Y.; Wilsey, S.; Lee, J. K. Comp. Theor. Chem. 1997, 398-399, 169.

(77) Björn, O. R. In Advances in Chemical Physics; Lawley, K. P., Ed. 2007; p 399.

(78) Robb, M. A.; Bernardi, F. New Theoretical Concepts for Understanding Organic Reactions; Bertran, J., Czismadia, I. G., Eds. Kluwer: Dordrecht, 1989.

(79) Bernardi, F.; Olivucci, M.; McDouall, J. J. W.; Robb, M. A. J. Chem. Phys. 1988, 89, 6365.

(80) Bachrach, S. M. Computational Organic Chemistry; WileyInterscience: Hoboken, NJ, 2007.

(81) Andersson, K.; Malmqvist, P. A.; Roos, B. O.; Sadlej, A. J.; Wolinski, K. J. Phys. Chem. 1990, 94, 5483.

(82) Andersson, K.; Malmqvist, P.-A.; Roos, B. O. J. Chem. Phys. 1992, 96, 1218

(83) Tao, H.-R.; Fang, D.-C. Theor. Chem. Acc. 2008, 121, 91.

(84) Lowe, J. P. J. Chem. Educ. 1974, 51, 785. 
(85) Garrais, S.; Turkington, J.; Goldring, W. P. D. Tetrahedron 2009, $65,8418-8427$.

(86) Coleman, R. S.; Lu, X.; Modolo, I. J. Am. Chem. Soc. 2007, 129, $3826-3827$.

(87) Yin, N.; Wang, G.; Qian, M.; Negishi, E. Angew. Chem., Int. Ed. 2006, 45, 2916-2920.

(88) Holland, D.; Stoddart, J. F. J. Chem. Soc., Perkin Trans. 1 1983, $1553-1571$. 